\title{
Feedback from Active Galactic Nuclei in Galaxy Groups
}

\author{
Dominique Eckert $^{1, *,+} \mathbb{D}$, Massimo Gaspari ${ }^{2,3,+} \mathbb{D}$, Fabio Gastaldello ${ }^{4,+} \mathbb{D}$, Amandine M. C. Le Brun ${ }^{5,+} \mathbb{D}$ \\ and Ewan $O^{\prime}$ Sullivan $6,+(D)$
}

Citation: Eckert, D.; Gaspari, M.; Gastaldello, F.; Le Brun, A.M.C.; O'Sullivan, E. Feedback from Active Galactic Nuclei in Galaxy Groups. Universe 2021, 7, 142. https:// doi.org/10.3390/universe7050142

Academic Editor: Francesco Shankar

Received: 31 March 2021

Accepted: 29 April 2021

Published: 11 May 2021

Publisher's Note: MDPI stays neutral with regard to jurisdictional claims in published maps and institutional affiliations.

Copyright: (c) 2021 by the authors. Licensee MDPI, Basel, Switzerland. This article is an open access article distributed under the terms and conditions of the Creative Commons Attribution (CC BY) license (https:/ / creativecommons.org/licenses/by/ $4.0 /)$.
1 Department of Astronomy, University of Geneva, Ch. d'Ecogia 16, CH-1290 Versoix, Switzerland

2 INAF-Osservatorio di Astrofisica e Scienza dello Spazio di Bologna, Via Piero Gobetti 93/3, I-40129 Bologna, Italy; massimo.gaspari@inaf.it

3 Department of Astrophysical Sciences, Princeton University, 4 Ivy Lane, Princeton, NJ 08544, USA

4 IASF-Milano, INAF, Via A. Corti 12, I-20133 Milano, Italy; fabio.gastaldello@inaf.it

5 LUTh, UMR 8102 CNRS, Observatoire de Paris, PSL Research University, Université Paris Diderot, 5 Place Jules Janssen, 92190 Meudon, France; amandine.le-brun@obspm.fr

6 Center for Astrophysics, Harvard \& Smithsonian, 60 Garden Street, Cambridge, MA 02138, USA; eosullivan@cfa.harvard.edu

* Correspondence: Dominique.Eckert@unige.ch

$+\quad$ All authors contributed equally to this work.

\begin{abstract}
The co-evolution between supermassive black holes and their environment is most directly traced by the hot atmospheres of dark matter halos. The cooling of the hot atmosphere supplies the central regions with fresh gas, igniting active galactic nuclei (AGN) with long duty cycles. Outflows from the central engine tightly couple with the surrounding gaseous medium and provide the dominant heating source preventing runaway cooling by carving cavities and driving shocks across the medium. The AGN feedback loop is a key feature of all modern galaxy evolution models. Here, we review our knowledge of the AGN feedback process in the specific context of galaxy groups. Galaxy groups are uniquely suited to constrain the mechanisms governing the cooling-heating balance. Unlike in more massive halos, the energy that is supplied by the central AGN to the hot intragroup medium can exceed the gravitational binding energy of halo gas particles. We report on the state-of-the-art in observations of the feedback phenomenon and in theoretical models of the heating-cooling balance in galaxy groups. We also describe how our knowledge of the AGN feedback process impacts galaxy evolution models and large-scale baryon distributions. Finally, we discuss how new instrumentation will answer key open questions on the topic.
\end{abstract}

Keywords: black holes; galaxy groups; elliptical galaxies; intragroup medium/plasma; active nuclei; X-ray observations; hydrodynamical and cosmological simulations

\section{Introduction}

Structure formation in the Universe operates as a bottom-up process in which small halos formed at high redshift progressively merge and accrete the surrounding material to form the massive halos we see today [1]. Given the evolution of the halo mass function, the peak of the mass density in the current Universe occurs in halos of $\sim 10^{13} M_{\odot}$ 一the galaxy group regime. At the current epoch, galaxy groups are the building blocks of the structure formation process and, thus, they occupy a key regime in the evolution of galaxies. Typical $L_{\star}$ galaxies exist within groups rather than within isolated halos [2]. The galaxy stellar mass function exhibits a cut-off at $M_{\star} \sim 10^{11} M_{\odot}$ [3], corresponding to the central galaxies of galaxy groups, brightest group galaxies (BGGs). Abundance matching studies show that the star formation efficiency reaches a maximum at $M_{h} \sim 10^{12} M_{\odot}$ and decreases at both higher and lower masses [4-6]. At the high-mass end, non-gravitational energy input is needed to quench star formation and reproduce the shape of the stellar mass function [7].

Feedback from active supermassive black holes (SMBH) is currently the favored mechanism for regulating the star formation activity in massive galaxies, explaining the observed 
shape of the stellar mass function, and quenching catastrophic cooling flows. Outflows and jets from the central active galactic nuclei (AGN) interact with the surrounding hot intragroup medium (IGrM) and release a large amount of energy, which prevents the gas reservoir from cooling efficiently and fueling star formation (for previous reviews, see McNamara and Nulsen [8]; Fabian [9]; Gitti et al. [10]; Gaspari et al. [11]). All of the modern galaxy evolution models include a prescription for AGN feedback to reproduce the shape of the galaxy luminosity function and the halo baryon fraction (see the companion review by Oppenheimer et al.). Earlier attempts at reproducing these observables with supernova feedback resulted in catastrophic cooling and largely overestimated the stellar content of groups (e.g., [12]). State-of-the-art cosmological simulations all implement sub-grid prescriptions for AGN feedback, either in the form of thermal, isotropic feedback, or in the form of mechanical, directional feedback. The adopted feedback scheme strongly affects the gas properties of galaxy groups. Indeed, strong feedback raises the entropy level of the surrounding gas particles, which can lead to a global depletion of baryons in group-scale halos. Cosmological simulations are now facing the important challenge of reproducing at the same time realistic galaxy populations and gas properties.

The imprint of AGN feedback is most easily observed through high-resolution Xray observations of nearby galaxy groups and clusters. Bubbles of expanding energetic material carve cavities in the gas distribution, spatially coinciding with energetic AGN outflows that are traced by their radio emission. Supersonic outflows also drive shock fronts permeating the surrounding IGrM and distributing heat across the environment. In parallel, extended $\mathrm{H} \alpha$ nebulae demonstrate the existence of efficient gas cooling from the hot phase, thereby feeding the central SMBH. Recent ALMA observations at millimetric wavelengths also provide evidence for large amounts of cool gas at the vicinity of the $\mathrm{SMBH}$. Thus, multi-wavelength observations of the cores of nearby massive structures allow us to investigate, in detail, the balance between gas cooling and AGN heating.

While a great deal of attention has been devoted to studying these phenomena in the most massive nearby clusters, such as Perseus (see [8] for a review), observations of similar quality only exist for a handful of groups, such as NGC 5044 [13] and NGC 5813 [14]. Detailed observations of galaxy groups are crucial for our understanding of feedback processes, as the physical conditions differ from those of galaxy clusters in several important ways. First and foremost, the ratio of feedback energy to gravitational energy is different from that of clusters. The AGN energy input is sometimes parameterized as $\dot{E}_{\text {feed }} \approx \epsilon_{f} \epsilon_{r} \dot{M}_{\mathrm{BH}} c^{2}$ with $\epsilon_{r} \sim 10 \%$ the energy output of the BH and $\epsilon_{f}$ the coupling efficiency between the $\mathrm{BH}$ outflows and the surrounding medium. On the other hand, the gravitational binding energy is a strong function of halo mass, $E_{\mathrm{bind}} \propto M_{h}^{2}$. If the coupling efficiency only depends on the physical properties of the gas and the feedback loop has a long duty cycle, then the total integrated feedback energy $E_{\text {feed }}$ becomes comparable to $E_{\text {bind }}$ or even exceeds it in group-scale halos, whereas it remains substantially lower in the most massive systems. Similarly, the radius inside, which non-gravitational energy dominates over gravitational energy, is comparably larger in group-scale halos. The impact of AGN in groups is spread over much larger volumes and it can even lead to a depletion of baryons within the virial radius. On top of that, the radiative cooling function experiences a transition of regime between the temperature range of clusters and that of groups. For temperatures that are greater than $\sim 3 \mathrm{keV}$, the plasma is almost completely ionized and the Bremsstrahlung process dominates. For temperatures of $\sim 1 \mathrm{keV}$, line cooling dominates, which makes the radiative losses comparably more important. Therefore, the radiative cooling time can become much shorter than the Hubble time, even at relatively low gas densities, and the supply of gas to the SMBH can be sustained more easily. For all of these reasons, studying the feedback loop across a wide range of halo masses is necessary for informing our theoretical models.

Here, we review the current state of the art in our knowledge of the AGN feedback process in the specific case of galaxy groups. For the purpose of this review, we define galaxy groups as galaxy concentrations with halo masses in the range $10^{13}-10^{14} M_{\odot}$ and 
with an X-ray bright intragroup medium (IGrM). Such masses correspond to virial temperatures of $\sim 0.5-2 \mathrm{keV}$. Most of the processes that are discussed in this review are also relevant in the case of $\mathrm{X}$-ray bright isolated elliptical galaxies and massive spirals with $k T \sim 0.3-0.5 \mathrm{keV}$. Whenever it is appropriate, we will discuss halos of lower masses as well. The paper is organized, as follows. In Section 2, we describe why AGN feedback is presently thought to be a key ingredient in the evolution of galaxy groups and how invoking AGN feedback can solve a number of overarching issues in galaxy evolution. In Section 3, we review the current observational evidence for AGN feedback in nearby galaxy groups, with our main focus on observations at $X$-ray and radio wavelengths and additional information coming from millimeter and $\mathrm{H} \alpha$ observations. Section 4 summarizes the theoretical framework that was put into place to interpret the heating/cooling cycle and the main physical processes involved, with a specific focus on the galaxy group regime. Section 5 discusses how AGN feedback is implemented in modern cosmological simulations and its impact on the evolution and large-scale distribution of the baryonic component of the Universe. We conclude our review in Section 6 with a short presentation of the most relevant upcoming experiments and their expected contribution to the field.

\section{The Need for AGN Feedback in Galaxy Evolution}

Even though the first indications that AGN feedback could be one of the missing elements in theories of galaxy formation and evolution are already nearly half a century old [15-17], theoretical and observational studies of the process are still in their infancy. In the last fifteen years, AGN feedback has emerged as the most promising solution to a number of overarching problems in galaxy formation and evolution in both semi-analytic models and cosmological hydrodynamical simulations (e.g., [18-22]). In short, the main issues are: cosmic 'downsizing' [23], i.e., the observation that the majority of both star formation and AGN activity took place before redshift $z \sim 1$ (e.g., [24,25]), the shape of the galaxy stellar mass function at the high-mass end, the gas content of massive galaxies, groups and clusters, the absence of a cooling flow, the deviations from self-similarity of gas scaling relations, and the entropy floor (the latter three are discussed in detail in Section 3.1). AGN feedback progressively appeared to be a promising solution to solve each of these problems separately, eventually leading to the realization that these issues could in fact be seen as different facets of the same problem (e.g., $[19,26,27])$. In this section, we highlight the main reasons why AGN feedback plays a central role in galaxy evolution models, specifically at the scale of galaxy groups.

\subsection{The Shape of the Galaxy Stellar Mass Function}

White and Rees [17] presented one of the first models of galaxy formation in a cosmological context (see also [16,28]). The authors proposed a two-stage process in which galaxies form via radiative cooling of the baryons within halos that had already formed via gravitational collapse of the collisionless dark matter. The authors also argued that an additional non-gravitational process, called feedback, was needed to avoid the overproduction of faint galaxies as compared to observations. Indeed, in that model, the galaxy stellar mass function would simply be a scaled-down version of the dark matter halo mass function (see the discussion in, e.g., [26] illustrated by their Figure 1 and Figure 1 of this review). Later studies pointed out that the scaled halo mass function model overpredicts the abundance of the most massive galaxies when compared to observations (e.g., [26,29-35]). In short, galaxy formation is fundamentally an inefficient process as only a small fraction of the Universe's baryons are in the form of stars (about 10 per cent; e.g., [36-38]) and the star formation efficiency strongly depends on halo mass. Thus, the mass of the dark matter halo plays a fundamental role in shaping the galaxies that it contains. Observational evidence for this halo-mass dependency of the efficiency of galaxy formation was first obtained using galaxy group catalogues $[39,40]$. 


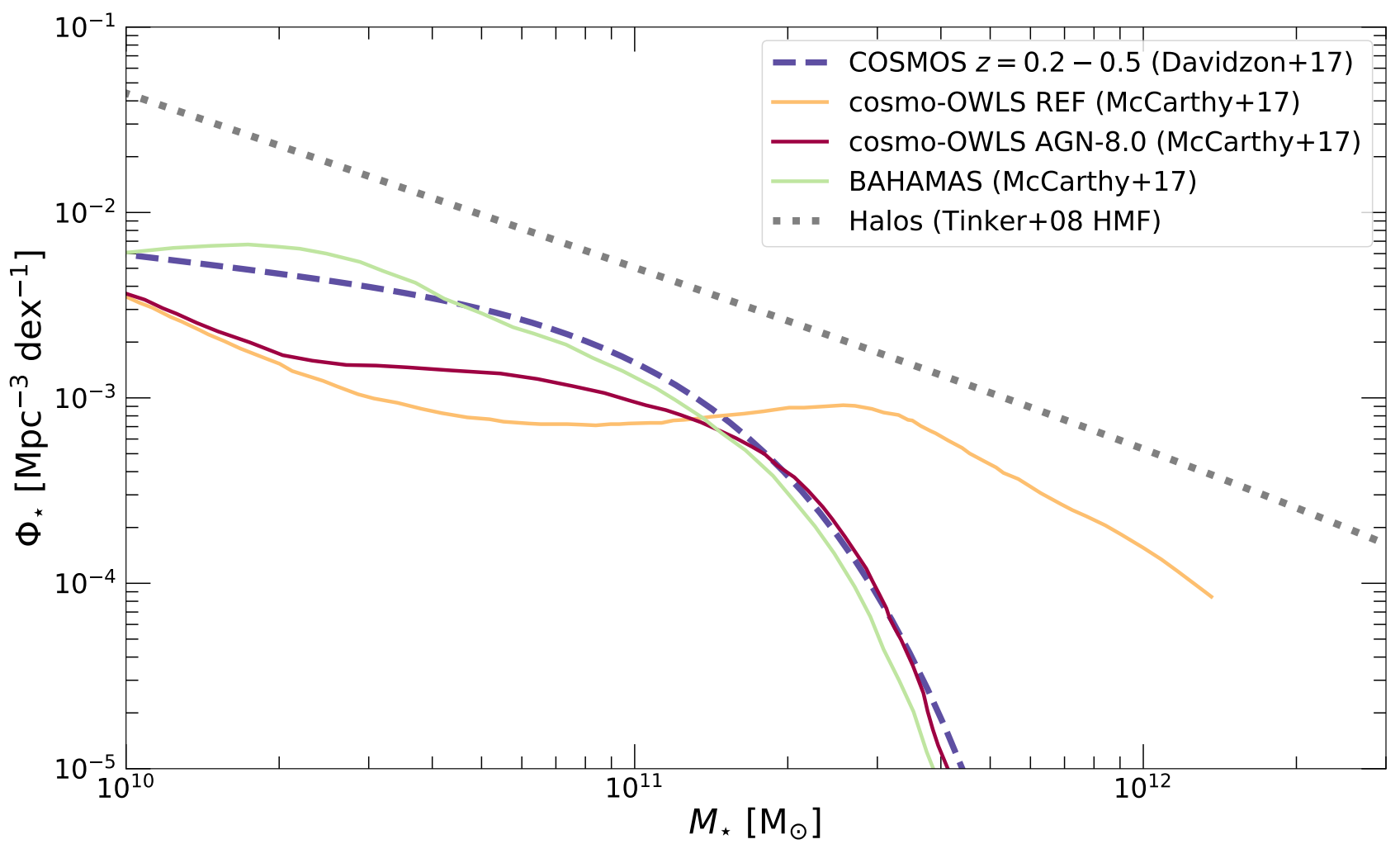

Figure 1. Galaxy stellar mass function (GSMF) $\Phi_{\star}\left(M_{\star}\right)=\frac{d N}{d M_{\star} d V}$ in observations and simulations. The dashed purple curve shows the double-Schechter fit to the local GSMF measured in the COSMOS survey [3]. The solid curves show the predictions of the cosmo-OWLS/BAHAMAS model [12,41] in the case with only stellar feedback (REF, yellow), cosmoOWLS with AGN feedback (AGN-8.0, maroon), and the latest BAHAMAS model (green), in which the feedback model was tuned to reproduce jointly the GSMF and the gas fraction. For comparison, the dotted gray curve shows the Tinker et al. [42] halo mass function in Planck cosmology scaled by $f_{b}=\Omega_{b} / \Omega_{m}$, which highlights what one expects to see in the case in which each halo is populated by a galaxy with $M_{\star}=f_{b} M_{h}$.

To illustrate the first point, in Figure 1 we show the local galaxy stellar mass function (GSMF) $\Phi_{\star}\left(M_{\star}\right)$ that is measured within the COSMOS survey [3] modeled using a double Schechter function. The dashed line shows the Tinker et al. [42] halo mass function with the masses scaled by the universal baryon fraction $f_{b}=\Omega_{b} / \Omega_{m}$, which produces the GSMF one would expect to see if every halo was populated by a single galaxy and all the available baryon content had been converted into stars. The observed GSMF vastly differs from the scaled halo mass function both in shape and normalization. The lower normalization implies that the star formation efficiency is much less than $100 \%$, whereas the steep decline at high masses shows that the growth of galaxies does not follow the structure formation process. Attempts at reproducing the shape of the GSMF with feedback from supernovae and star formation were unsuccessful, as the injected energy was insufficient to offset cooling and regulate the star formation efficiency [26]. In Figure 1, we compare the observed GSMF with the predictions of hydrodynamical cosmological simulations from the cosmo-OWLS and BAHAMAS suites [12,41] implementing several prescriptions for baryonic physics. The cosmo-OWLS run, including cooling, star formation, and stellar feedback (labelled REF) suffers from overcooling, and, thus, it overpredicts the observed abundance of massive galaxies $\left(M_{\star} \gtrsim 10^{12} M_{\odot}\right)$ by several orders of magnitude. Conversely, including AGN feedback allows the model to closely reproduce the abundance of massive galaxies. AGN feedback is implemented by releasing a fraction of the rest-mass energy of cooling gas particles within the surrounding environment, which reheats the gas and regulates the star formation efficiency (see Section 5.1 for details). The effect of AGN 
feedback kicks in around $M_{\star} \sim 2-3 \times 10^{11} M_{\odot}$ corresponding to the stellar masses of BGGs, which highlights the crucial role that is played by the galaxy group regime.

Following the early works highlighting the discrepancy between the observed GSMF and the structure formation theory, it took over 30 years to pinpoint the most likely source of feedback. Early models of non-gravitational heating invoked a 'pre-heating' of the baryonic content before the epoch of formation of massive haloes, but did not specify the source of the energy injection (e.g., [31,43,44]). Because these models cannot predict the impact on the galaxy population, but only on the intra-group medium, they are not discussed any further here (but, see Section 3.1 for a detailed discussion). Models implementing gas cooling without feedback were able to reproduce the breakdown of self-similarity that was observed in X-ray selected samples, but this came at the price of greatly overpredicting the abundance of galaxies (e.g., [45-50] for a review). In the late 1990s and early 2000s, teams working with both semi-analytic models and hydrodynamical simulations, who had started to model the effects of cooling, star formation, and stellar feedback, came to the realization that supernova heating, while being a suitable explanation for the inefficiency of galaxy formation at the low-mass end, could neither solve the remaining problem with the high-mass end of the galaxy stellar mass function (if anything it made it worse; see e.g., Menci and Cavaliere [51]; Bower et al. [52]; Benson et al. [26]) nor reproduce the properties of the gas in massive galaxies, groups, and clusters (e.g., [53-55]). Indeed, the amount of energy that is injected by supernovae is insufficient to eject gas from the potential wells of groups and clusters, even if one assumes that the feedback is one hundred per cent efficient (e.g., [56-58]), and such efficiencies were in conflict with contemporary observations of galactic outflows (e.g., [59]). Heating from thermal conduction was also investigated (e.g., [26,60-62]) and eventually ruled out, as it required that the conduction coefficient should exceed the Spitzer rate expected for a fully ionized plasma. By contrast, the energy that is injected by the supermassive black holes at the center of galaxies is, in principle, sufficient to eject gas from the potential wells of groups and clusters (e.g., $[18,26,63]$ ). Additionally, the existence of a feedback loop between supermassive black holes and galaxy formation provides an attractive solution to explain the observed correlation between galaxy and black hole properties (see Sections 2.2 and 5.3 for details). Galaxy groups are the best astrophysical laboratories for studying the impact of various feedback mechanisms, since they have managed to retain enough hot gas to allow for a study of the impact of feedback on the IGrM, while, at the same time, representing a transitional regime for the stellar properties of galaxies. We will discuss this point in more detail in the remainder of this review.

\subsection{Co-Evolution between Black Hole Mass and Galaxy Properties}

Since the late 1990s, it has become clear that central SMBH co-evolves with the properties of their host galaxies. Thanks to the increasingly precise measurements of $\mathrm{SMBH}$ masses obtained through spatially resolved dynamical measurements of stars and gas at the BH's vicinity, it is now well established that the vast majority of galaxies host a central SMBH with a mass that correlates with the properties of its host galaxy (see [64] for an extensive review). The SMBH mass was found to correlate with a galaxy's near-infrared luminosity $L_{K}$, i.e., with the galaxy's integrated stellar content, and with the velocity dispersion $\sigma_{v}$ of the stars in the bulge, which is often used as a proxy for the halo mass [64-70]. SMBH masses scale with galaxy properties as $M_{\mathrm{BH}} \propto \sigma_{\mathrm{e}}^{4.5}$ and $M_{\mathrm{BH}} \propto L_{K}^{1.1}$. While it is still unclear whether the relations between SMBH mass and galaxy properties are fundamental or derive from correlations between hidden variables, the existence of these scaling relations implies that the processes leading to the growth of $\mathrm{BH}$ are tightly linked with the evolution of the host halo. Widespread AGN feedback provides a natural explanation for the existence of $\mathrm{SMBH}$ scaling relations $[7,18,71]$. The radiative and mechanical energy output of AGN outbursts can, in principle, be sufficient to expel cold gas away from galaxy bulges, thereby, at the same time, regulating the AGN accretion rate and the star formation activity of the host galaxy. Feedback from AGN has the potential 
to directly link the properties of supermassive black holes and their host galaxies. The coupling of the energy released by the formation of the supermassive black hole to the surrounding forming galaxy should lead to a relationship that is close to the observed $M_{\mathrm{BH}}-\sigma_{\mathrm{e}}$ relation $[63,72]$. The generality of these arguments imply that this result may be reasonably independent of the specific details of the feedback model.

While the $M_{\mathrm{BH}}-L_{K}$ and $M_{\mathrm{BH}}-\sigma_{\mathrm{e}}$ relations are now well established, the typical intrinsic scatter of optical/stellar relations remains substantial (even in early-type galaxies), $\epsilon \sim 0.4-0.5$ dex, especially for stellar luminosities and masses (e.g., Saglia et al. [73]). Moreover, optical extraction radii are necessarily limited to galactic half-light radii $R_{\mathrm{e}}$ $(\sim 2-5 \mathrm{kpc})$, neglecting the key role of the host halo of the group. It has been suggested that $\mathrm{SMBH}$ properties are fundamentally linked with the mass of the host halo [74,75], and that the $M_{\mathrm{BH}}-L_{K}$ and $M_{\mathrm{BH}}-\sigma_{v}$ relations arise as a byproduct from the scaling relations between halo mass and optical galaxy properties. Several recent studies seem to confirm that the mass of the central SMBH is more tightly related to the temperature of the host gaseous halo, i.e., the global gravitational potential and hot-halo processes [76-78]. We discuss this point, in detail, in Section 5.3.

\section{Observational Evidence}

\subsection{X-ray Observations}

\subsubsection{Feedback-Induced Hydrodynamical Features}

The X-ray observatories in orbit for the past 20 years, Chandra and XMM-Newton, have revolutionized our understanding of the cores of relaxed galaxies, groups, and clusters, which show a highly peaked X-ray emission from a hot interstellar medium whose radiative cooling time is often less than 1 Gyr. Soon after the launch of XMM-Newton and Chandra it was realized that the gas in the central regions of nearby groups and clusters does not efficiently cool from the X-ray phase, condense, and flow toward the center, as expected from the original 'cooling flow' model [79]. Spectroscopic observations with Chandra and XMM-Newton have established that there is little evidence for emission from gas cooling below $\sim T_{\text {vir }} / 3$ [80-82]. Precisely where the gas should be cooling most rapidly, it appears not to be cooling at all. This effect is known as the 'cooling flow problem' (e.g., [83]).

Therefore, a compensating heat source must resupply the radiative losses, and many possibilities have been proposed, including thermal conduction (e.g., [84]), energy released by mergers (e.g., [85,86]), or by supernovae (e.g., [87]); see Section 2.1. However, feedback from the central AGN was rapidly established as the most appealing solution to the problem. There is, in fact, clear observational evidence for AGN heating as the majority of brightest cluster galaxies of cool-core clusters and groups host a radio loud AGN (e.g., [88,89]) and, following the launch of Chandra, disturbances, such as shocks, ripples, and cavities, have been found in the central atmospheres of many clusters, groups, and elliptical galaxies (e.g., [90-98]). The cavities, which appear as X-ray surface brightness depressions, have been interpreted as bubbles of low-density relativistic plasma inflated by radio jets, displacing the thermal gas and causing $P d V$ heating (e.g., [99]). Weak shocks that are associated with outbursts, long expected in models of jet-fed radio lobes [100], were also finally detected in deep Chandra observations, for example, in M87 [93], Hydra A [101], and MS 0735+7241 [102]. The energies available from the AGN were found to be not only comparable to those that are needed to stop gas from cooling, but the mean power of the outbursts was well correlated with the radiative losses from the IGrM [103].

Given the lower surface brightness of groups, in the early days of the Chandra era, the study of AGN feedback in these systems did not progress at the same pace as for more massive clusters [8]. However, the situation has improved in more recent times, with a number of studies addressing sizable samples of groups and characterizing the cavities in their IGrM [104-107]. Table A1 in Appendix A presents a list of groups with known cavities being detected using high-resolution X-ray observations. Deep Chandra X-ray data are now available for a number of galaxy groups (HCG 62, NGC 5044, NGC 5813). In particular, Randall et al. [14] presented the results of a very deep (650 ks) observation 
of NGC 5813, the longest such observation available to date. In Figure 2, we show the Chandra image and temperature map of NGC 5813, revealing an impressive number of feedback-induced features. Multiple pairs of X-ray cavities can be observed on both sides of the nucleus, indicating that the system has undergone several consecutive AGN outbursts inflating powerful expanding bubbles. Two pairs of concentric shock fronts were discovered perpendicular to the jet axis. The passage of the shock fronts reheats the IGrM, as evidenced by the higher temperatures that were measured in the post-shock regions (see the right-hand panel of Figure 2).
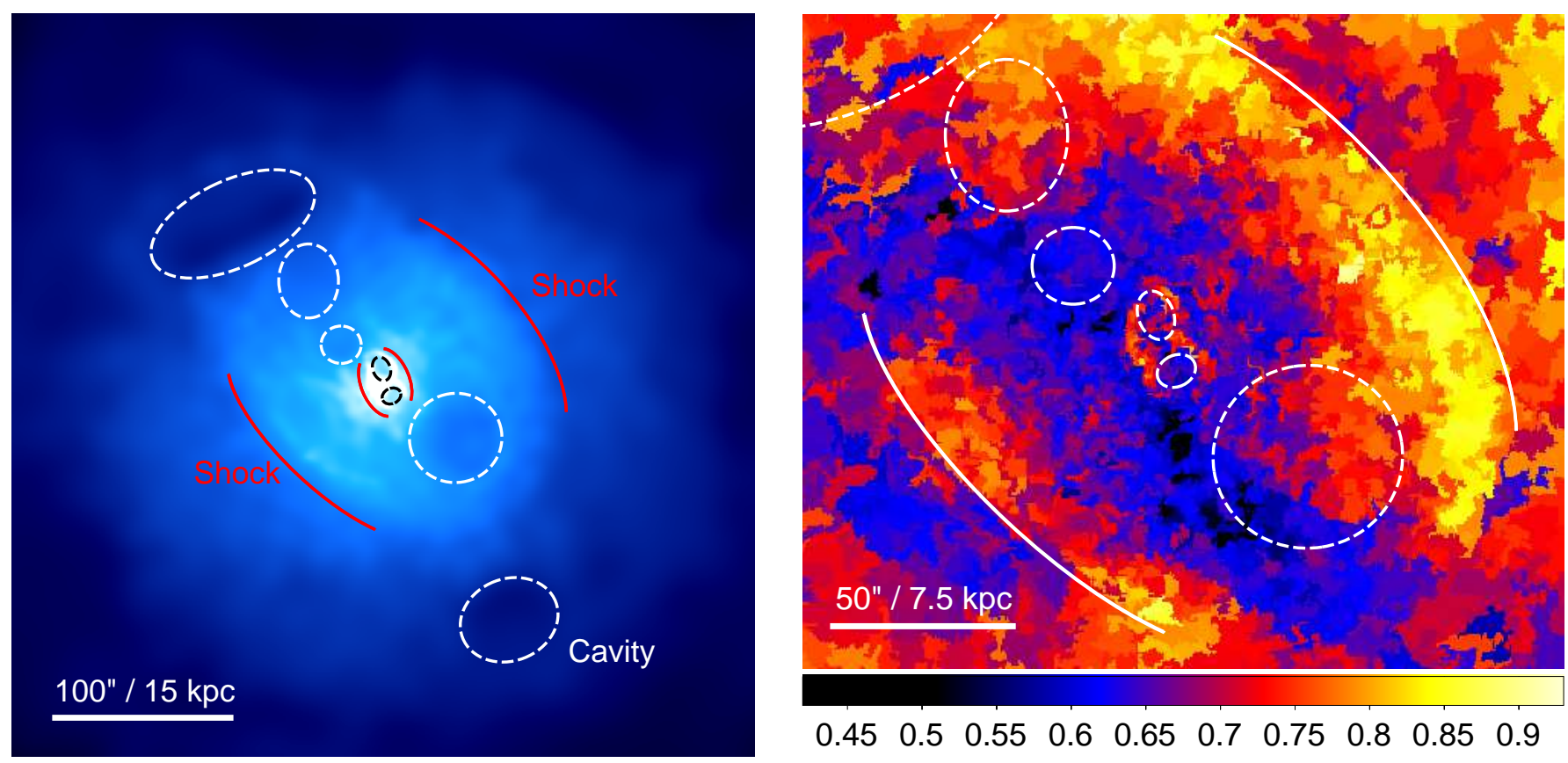

Figure 2. Cavities and shocks in the NGC 5813 galaxy group. The left panel shows an adaptively smoothed Chandra 0.5-2 keV image of the group, with cavities marked by dashed ellipses and two pairs of shock fronts by solid curved lines. The right panel shows a temperature map (in units of $\mathrm{keV}$ ) with the cavities and outer shock fronts marked. Note the shock-heated gas (red and yellow) behind the outer shock fronts and in the shocked rims of the innermost set of cavities. Images drawn from the Chandra Early-type Galaxy Atlas [108].

Because cavities are the most commonly detected feedback-related structures, AGN energy input is usually gauged from their properties, as briefly summarized below. The energy that is required to inflate radio bubbles creating cavities in the X-ray emitting gas is usually expressed as the enthalpy, i.e., the sum of the work done to carve out the cavity and the internal energy of the radio lobes:

$$
H=E_{\mathrm{int}}+p V=\frac{\gamma}{\gamma-1} p V
$$

where $p$ is the pressure of the surrounding IGrM, $V$ is the volume of the cavity, and $\gamma$ is the ratio of the specific heats of the plasma filling the cavities. If the plasma is relativistic, $\gamma=4 / 3$ and $H=4 p V$; if it is non-relativistic, $\gamma=5 / 3$ and $H=2.5 p V$. The exact composition of the cavities is still unknown, even though $X$-ray, radio ([109] and references therein), and SZ observations [110] suggest that it is likely a mixture of the two species. The age of the cavity is the other key physical quantity that can be estimated from observations. As summarized by Bîrzan et al. [92], several age estimators have been suggested: 
(a) the sonic time, i.e., the time that is required by the cavity to reach its projected distance $R$ at the speed of sound,

$$
t_{s}=R / c_{s}
$$

with $c_{s}=\left(\gamma k T / \mu m_{H}\right)^{1 / 2}, \mu$ the mean atomic weight of the plasma, and $m_{H}$ the proton mass;

(b) the refill time that is required by the gas to refill the displaced volume as the cavity rises upward,

$$
t_{\mathrm{ref}} \approx \sqrt{r / g}
$$

where $r$ is the radius of the cavity and $g=G M(<R) / R^{2}$ is the gravitational acceleration at the cavity position;

(c) the buoyancy time, i.e., the time that iis required for the cavity to rise buoyantly at its terminal velocity,

$$
t_{\text {buoy }}=R / v_{t} \approx R \sqrt{S C / 2 g V}
$$

where $V$ and $S$ are the volume and the cross-section of the cavity, respectively, and $C=0.75$ is the drag coefficient [111]

Cavity ages that are estimated using these three methods usually agree within a factor of two, with the buoyancy times typically in between the shorter sonic time and the longer refill time ([10] and references therein). Dividing the enthalpy $H$ by the characteristic timescale provides an observational estimate of the cavity power $P_{c a v}$. The cavity power is a lower limit to the mechanical power of the AGN, given the paucity of detected shocks and other possible sources of energy feedback, such as sound waves.

Operationally, estimating $P_{c a v}$ requires a measurement of the geometry and size of the cavity and the pressure of the surrounding ICM. The total cavity power can be compared with the gas luminosity inside the cooling radius, $L_{\mathrm{cool}}$, which needs to be balanced by the AGN mechanical feedback. $L_{\text {cool }}$ is usually defined as the total luminosity inside the regions where the cooling time is less than $7.7 \times 10^{9}$ yrs [112], although different thresholds exist in the literature (e.g., $3 \mathrm{Gyr}$, [113]). $L_{\text {cool }}$ is estimated by deprojecting the $\mathrm{X}$-ray temperature and emissivity profiles and computing the corresponding bolometric luminosity [96]. A number of possible biases and systematic errors can affect this apparently straightforward observational approach, as the detectability of cavities depends on the depth of the observation, the position of the cavity with respect to the plane of the sky, and uncertainties in the assumed geometry $([8,10,109]$ and the references therein). The exact impact of these observational uncertainties on the statistics of cavities in clusters and groups is yet to be quantified.

The $P_{\text {cav }}-L_{\text {cool }}$ relation has been investigated through the years in an increasing number of objects, ranging from ellipticals to groups and clusters $([10,109]$ and references therein). The general consensus is that the cavity power is enough to offset cooling given an average $4 \mathrm{pV}$ injected energy per cavity and that the jet mechanical power correlates well with the cooling luminosity. In Figure 3, we show the relation between cooling luminosity and cavity power from a compilation of literature measurements [104,106,112]; see Table A1. Here, the total cavity power for each system was computed by summing up the power of each individual cavity. While at the high-mass end, the data are broadly consistent with an enthalpy $H=4 p V$, being typical of heating by a relativistic plasma, in the group regime, the cavity power is substantially higher. To quantify this effect, we fitted the $L_{\text {cool }}-P_{\text {cav }}$ relation with a power law using PyMC3 [114]. The blue curve and shaded area show the best-fit relation, which reads

$$
\log \left(\frac{P_{\text {cav }}}{10^{43} \mathrm{erg} / \mathrm{s}}\right)=(0.41 \pm 0.09)+(0.70 \pm 0.05) \log \left(\frac{L_{\mathrm{cool}}}{10^{43} \mathrm{erg} / \mathrm{s}}\right)
$$

with an intrinsic scatter of $0.51 \pm 0.07 \mathrm{dex}$. The slope of the fitted relation is significantly shallower than unity, which would be the expected slope if the feedback efficiency is 
independent of the halo mass. At face value, this result implies that the feedback efficiency is higher in groups than in clusters, with the cavities injecting enough energy to overheat the cores and deplete the central regions from their gas content. We discuss this point, in detail, in Section 3.1.4.

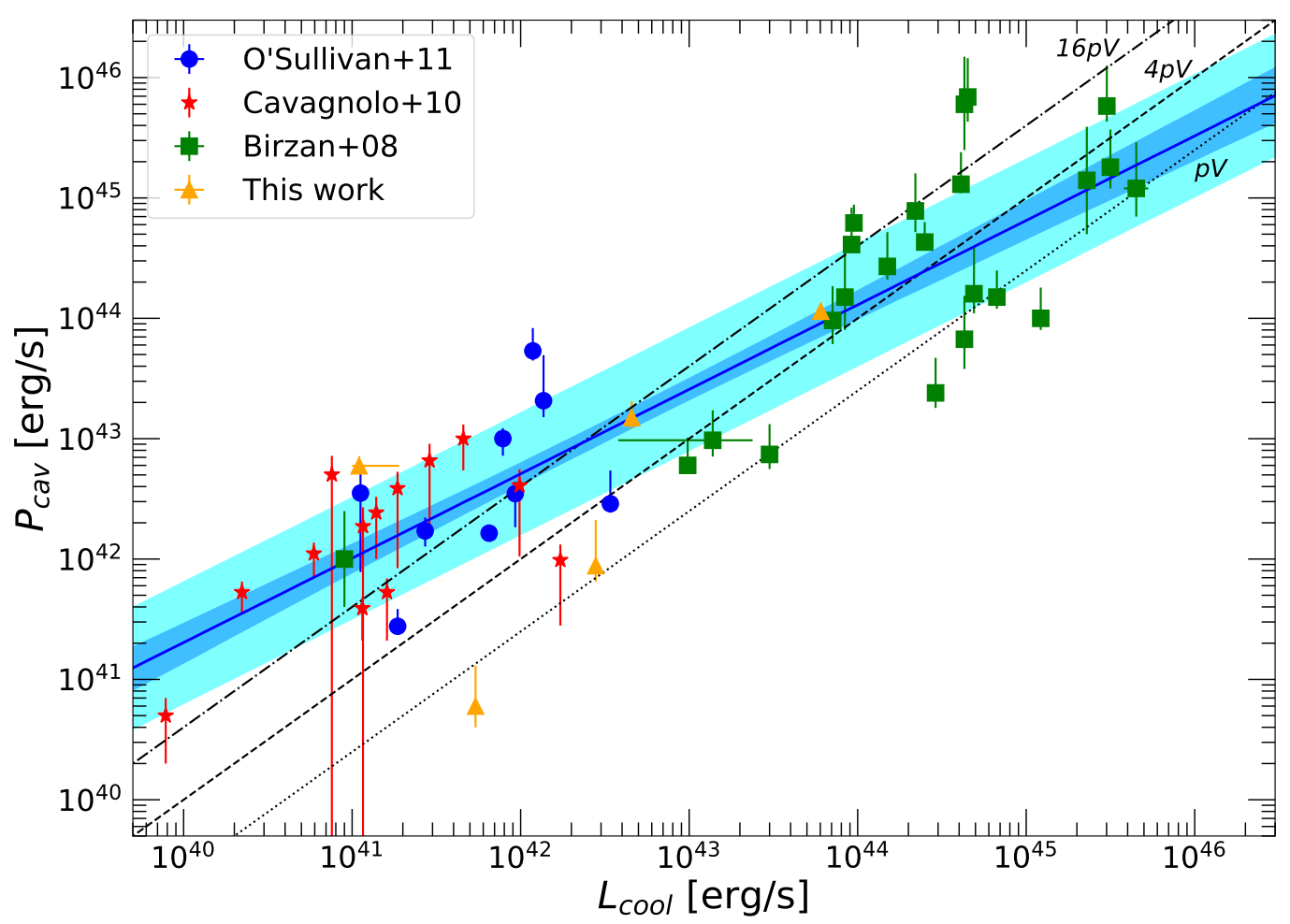

Figure 3. The relation between the luminosity within the cooling radius $\left(L_{c o o l}\right)$ and the power injected by the cavities assuming $H=4 p V\left(P_{c a v}\right)$ for several literature samples. The orange points were either recomputed for this work or collected from papers on individual objects; we refer to Table A1 for detailed references. The blue line shows a fit to the data using a power law with intrinsic scatter. The uncertainty on the fitted relation is indicated by the blue shaded area, whereas the cyan range indicates the intrinsic scatter around the relation.

The presence of shock fronts is another clear observational hydrodynamical feature that is caused by AGN feedback. The passage of a shock front compresses and heats the gas, raising its entropy and providing an effective heat input

$$
\Delta Q \approx T \Delta S=T \Delta \ln K
$$

with $K$ the entropy index usually quoted as entropy by X-ray astronomers (see Section 3.1.2). Because of their transient nature, single weak shocks fail to compensate for the radiative losses in a cool core, but the cumulative effect of multiple shocks can be relatively important (see, e.g., the discussion in [109]). This is again highlighted by the exemplar case of NGC 5813 (see Figure 2), where each set of three cavities has been associated with an elliptical shock measured at $1 \mathrm{kpc}, 10 \mathrm{kpc}$ and $30 \mathrm{kpc}$, respectively, with Mach numbers $\mathcal{M}$ in the range 1.17-1.78. Generally speaking, the detected shock fronts are weak, i.e., their Mach number falls in the range $\mathcal{M} \sim 1-2$ [115]. This range can be understood given the typical evolution of the Mach number as a function of the fundamental parameters, total energy, and duration, of the AGN outbursts (see, for example, the discussion in [116] and Section 4.2). The cumulative heating effect of these successive shock fronts is sufficient to offset cooling within the inner $30 \mathrm{kpc}$. The shock energy can be estimated as

$$
E_{s}=p_{1} V_{s}\left(p_{2} / p_{1}-1\right)
$$


where $p_{1}$ and $p_{2}$ are the pre- and post-shock pressures, respectively, and $V_{s}$ is the volume that is enclosed by the shock (e.g., [14]). Liu et al. [115] made an exhaustive search for groups and clusters with detected shocks and studied the dependence of the shock energies and related Mach numbers on the cavity enthalpies (see Figure 4). The shock energies span almost seven orders of magnitude from $4 \times 10^{61} \mathrm{erg} \mathrm{s}^{-1}$ in the cluster MS $0735+7421$ [102] to $10^{55} \mathrm{erg} \mathrm{s}^{-1}$ in NGC 4552 [117], with group-scale objects in the range $10^{56}-10^{59} \mathrm{erg} \mathrm{s}^{-1}$ and Mach numbers all in the range 1-2 with the exception of Centaurus A. The shock energy is similar to the cavity energy (see the right-hand panel of Figure 4), suggesting that shocks and cavities may play a comparable role in supplying mechanical energy that is provided by the AGN (with the balance between the two mainly driven by the duration of the outburst, e.g., $[116,118])$. Other heating mechanisms that are discussed in Section 4.2, such as turbulent heating, have yet to be explored observationally at the group scale.
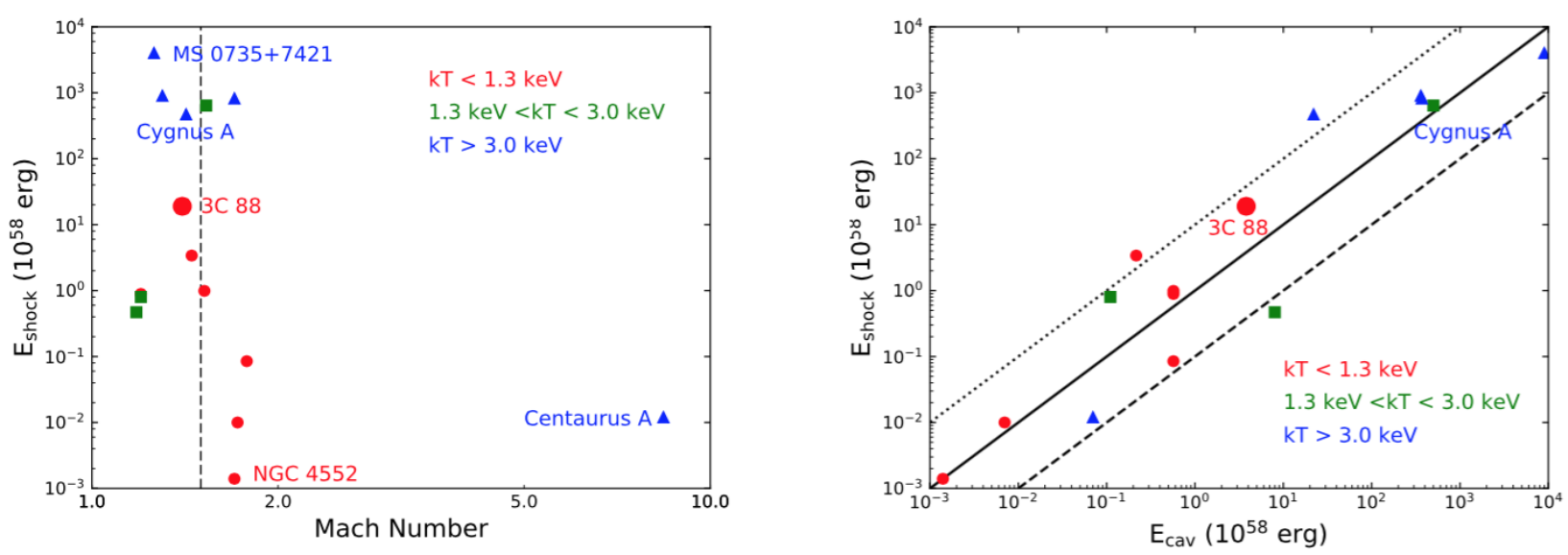

Figure 4. Shock energy versus Mach number (left panel) and shock energy versus cavity enthalpy (right panel) for groups and clusters with available shock energy in the literature. Figure reproduced from Liu et al. [115]. Objects with a red circle have $k T<1.3 \mathrm{keV}$, green square: $1.3<k T<3.0 \mathrm{keV}$ and blue triangles $k T>3.0 \mathrm{keV}$. The dashed line in the left panel marks $\mathcal{M}=1.5$ and the dotted, solid, dashed lines in the right panel represent $E_{\text {shock }} / E_{\text {cav }}=10,1,0.1$ respectively. The objects considered in the plots are: Hydra A [101,119], MS 0735+7421 [102], Centaurus A [120], Cygnus A [92,121], 3C 444 [122], M87 [118], Abell 2052 [123], 3C 310 [124], NGC 4552 [117], NGC 4636 [125], HCG 62 [126], and NGC 5813 [14].

\subsubsection{Non-Gravitational Feedback Energy and Entropy Profiles}

One of the earliest pieces of evidence for non-gravitational feedback energy came from observed deviations from the self-similar scaling relations that are driven only by gravity [127], in particular the deviation of the observed luminosity-temperature relation from the predicted $L \propto T^{2}$ [128-130]; see the companion review by Lovisari et al. on the scaling relations of galaxy groups. Advocating a minimum entropy in the pre-collapse intergalactic medium to break self-similarity was one of the first attempts at a solution [43,44], with the result of bending the relation from self-similar at the scale of massive clusters to a steeper slope at the scale of groups. It was recognized that a given entropy level could be reached through different thermodynamic histories, and that the key insight would have been given by the sequence of adiabats through which baryons evolve: the excess entropy could have been achieved prior accretion to the collapsed halo (the external scenario) or in the higher density medium after accretion (internal scenario (e.g., [131,132]). A third option was initially considered while realizing that cooling alone could remove low entropy gas from the centers of halos producing a similar effect to non-gravitational heating (e.g., [46,133]).

Ponman et al. [134] provided the first observational evidence by measuring the entropy $\left(S=T / n_{e}^{2 / 3}\right)$ at a fixed scaled radius $\left(0.1 r_{\text {virial }}\right)$, and showing that it does not follow the expected linear trend with temperature (see the left-hand panel of Figure 5). ROSAT surface brightness profiles were combined with Ginga mean temperatures under the assumption of isothermality to derive entropy profiles for 25 objects, six objects with $T<2 \mathrm{keV}$ 
(NGC4261, NGC2300, NGC533, HCG97, HCG94, and HCG62), and objects, like MKW4, MKW3, AWM4, and AWM7, all in the range $T=2-4 \mathrm{keV}$. The authors concluded that the observational data were consistent with a scenario involving an external preheating mechanism through supernova winds, thereby raising the central entropy and enriching the medium in heavy elements. They also established that preheating would have a broader impact on the general picture of structure formation, as, for example, the level and timing of the heating required could not violate the constraints from the Lyman- $\alpha$ forest (see also [135]).
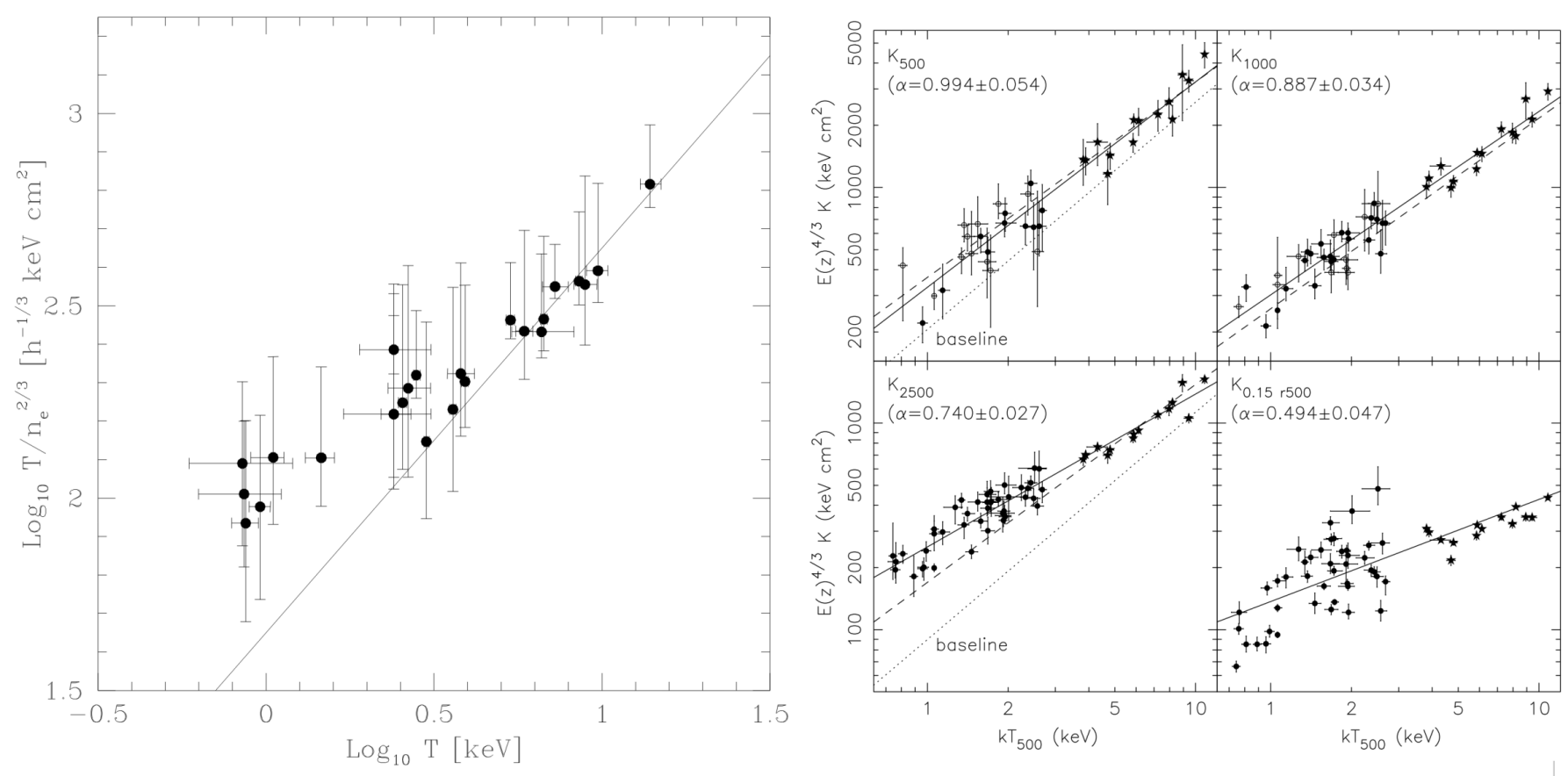

Figure 5. Left: The gas entropy at the fiducial radius of $0.1 R_{\text {virial }}$ as a function of the temperature for the 25 systems in the sample of Ponman et al. [134]. The solid line shows the relation obtained from numerical non-radiative simulations [136]. Figure reproduced from Ponman et al. [134], arXiv author's version. Right: The same relation at different radii for the groups analyzed in the sample of Sun et al. [137], together with the sample of clusters in Vikhlinin et al. [138], both being analyzed with Chandra data. Figure reproduced from Sun et al. [137].

A key improvement with respect to this early result was the ability to go beyond the assumption of isothermality by constraining the temperature profiles exploiting the combination of ROSAT and ASCA data [139,140]. These studies confirmed that low-mass systems exhibit higher scaled entropy profiles. However, they did not show the large isentropic cores that were predicted by simple preheating models (e.g., [141]) and the high entropy excess in galaxy groups was found to extend to large radii (as also shown by the ASCA analysis of [142]).

The Chandra and XMM-Newton results have made the observational picture clearer and more solid. In the comprehensive work that was done by [137], an archival sample of 43 groups observed with Chandra with a temperature range of $k T_{500}=0.7-2.7 \mathrm{keV}$ was analyzed, deriving detailed entropy profiles thanks to the superb spatial resolution of the satellite. The derived entropy-temperature scaling relations at six characteristic radii (30 kpc, $0.15 R_{500}, R_{2500}, R_{1500}, R_{1000}$, and $R_{500}$ ) show a large intrinsic scatter at small radii, but already at $R_{2500}$, the scatter reaches a value of $10 \%$ and remains the same beyond this point. When combined with similar observations in the galaxy cluster regime (the sample of [138]), the slope of the relation is found to gradually approach the self similar value, steepening from $0.740 \pm 0.027$ at $R_{2500}$ to $0.994 \pm 0.054$ at $R_{500}$ (see the right-hand panel of Figure 5). The entropy ratios that were calculated with respect to the baseline entropy 
profile expected from purely gravitational processes [143] confirm an excess entropy, which is a function of mass and radius, with groups having higher ratios at small radii. The weighted mean ratio for groups decreases from 2.2 at $R_{2500}$ to 1.6 at $R_{500}$. In general, the entropy profiles of groups have slopes $(0.7-0.8)$ that are flatter than the self-similar expectation from pure gravitational processes of 1.1. Deep observations of nearby poor clusters with Suzaku (RX J1159, Humphrey et al. [144]; Virgo, Simionescu et al. [145]; UGC 03957, Thölken et al. [146]) have shown that the entropy excess can extend all the way to the system's virial radius. Similar observations on a larger sample of groups are needed to determine whether the high entropy of galaxy groups is a general feature that is linked to the AGN feedback phenomenon.

The XMM-Newton study that wsa performed by [147] on a sample of 29 groups based on the two-dimensional XMM-Newton Group Survey, 2DXGS $[148,149]$ supplemented by groups from the sample of Mahdavi et al. [150] divided the objects into cool core (CC) and non-cool core (NCC) objects on the basis of the presence of a temperature gradient in the core, the first time this had been done for galaxy groups. The slope of the scaling relations of the entropy with temperature, incorporating the cluster sample of Sanderson et al. [151], at $0.1 R_{500}$ has a slope of $0.79 \pm 0.06$ consistent with the results of Sun et al. [137]. The entropy profiles of NCC groups show greater scatter than the CC sub-sample, and they have higher central entropies, in qualitative agreement with the results at the cluster scale (e.g., [152]). The excess entropy with respect to the baseline expected from gravitational processes cannot be reproduced by simple theoretical models of entropy modification, such as pure pre-heating or pure cooling, and the required mechanism should provide increasingly large entropy shifts for higher entropy gas.

Another significant advance in the study of entropy profiles of group-scale objects has been provided by the work of Panagoulia et al. [153], which analyzed the entropy profiles of 66 nearby groups and clusters drawn from a volume-limited sample of 101 objects that were assembled from the NORAS and REFLEX catalogues. The study pointed out that the flattening of the entropy profiles at small radii found in previous studies [152] was mainly a matter of resolution and it could be affected by the presence of multi-temperature gas. In particular, for nearby groups, a broken power law model provides the best description of the entropy profiles, with an inner slope of 0.64 within the central $20 \mathrm{kpc}$. This finding was confirmed in the recent studies of Hogan et al. [154] and Babyk et al. [155], who found that the behavior of the entropy profiles in the inner regions of relaxed clusters and groups can be well described by a broken power law with $K(r) \propto R^{2 / 3}$, a break around $100 \mathrm{kpc}$ $\left(\sim 0.1 R_{2500}\right)$ and an outer slope of $\sim 1.1$ matching the predictions of gravitational collapse models [143]. Because the cooling time is $\propto K^{3 / 2}$, the non-existence of an entropy floor affects the interpretation of the SMBH feeding and feedback processes, as we will discuss in Section 3.1 .3 and Section 4.

\subsubsection{Thermal Instability Timescale Profiles}

In the past decade, a substantial amount of work has been dedicated to understanding the triggering of AGN feedback in galaxy groups and clusters. The energy injected by the central AGN and the cooling of the IGrM appear to be closely balanced over the longterm, as we will review in Section 4. This reflects a tight relation between cooling/feeding (Section 4.1) and heating/feedback (Section 4.2) processes. Initial works suggested that the onset of thermal instability in hot halos and the triggering of runaway cooling can be expressed in terms of the ratio of the cooling time to the free-fall time [156-160]. The cooling time is usually expressed as the ratio of the thermal energy to radiative cooling rate,

$$
t_{\mathrm{cool}}=\frac{(3 / 2) n k_{\mathrm{B}} T}{n_{\mathrm{e}} n_{\mathrm{i}} \Lambda} \approx \frac{3 k_{\mathrm{B}} T}{n_{\mathrm{e}} \Lambda}
$$

with $\Lambda$ the cooling function (see Figure 10 ) and $n_{\mathrm{i}} \approx n_{\mathrm{e}}$ the IGrM ion number density. The free-fall time describes the timescale that is necessary for a gas particle to directly fall to the bottom of the potential well, 


$$
t_{\mathrm{ff}}=[2 R / g(R)]^{1 / 2}
$$

with $g(R)$ the local gravitational acceleration. If $t_{\text {cool }} \gg t_{\mathrm{ff}}$, the gas particle is losing internal energy too slowly and radial oscillations are eventually damped. Conversely, as $t_{\text {cool }}$ becomes comparable to $t_{\text {ff }}$, the gas rapidly loses pressure support, developing runaway thermal instability, and eventually sinking radially onto the central galaxy and SMBH. This 'classical' thermal instability (and related TI-ratio) has been also referred to as 'raining' (Gaspari et al. [156]) or 'precipitation' [159], being analogous to early physics studies that are based on analytical approximations (e.g., Field [161]).

The observational constraints on thermal instability can be investigated by studying the radial profiles of such IGrM timescales. In the absence of cooling and non-gravitational heating $\left(t_{\text {cool }} \gg 1 \mathrm{Gyr}\right)$, the cooling time follows a baseline universal profile set by the structure formation process $[143,162]$. Deviations from the baseline profile occur in the central regions, where $t_{\text {cool }} \ll 1 / H_{0}$ and radiative cooling losses can no longer be neglected. The left-hand panel of Figure 6 shows the cooling time profiles for a large sample of galaxy clusters and groups from the ACCEPT database $[159,163]$. All of the systems exhibiting evidence of multi-phase gas (e.g., $\mathrm{H} \alpha, \mathrm{CO}$ ) show a floor set by the threshold $t_{\text {cool }} / t_{\text {ff }} \sim 10$ 30 , such that, on average, the IGrM does not experience runaway cooling. Figure 6 (right panel) includes 40 galaxy groups and massive early-type galaxies with deep Chandra observations [155], showing that the ratio of cooling time to free-fall time reaches a floor at $t_{\text {cool }} / t_{\text {ff }} \sim 10$. In the inner regions where the entropy rises approximately as $K \propto R^{2 / 3}$, $t_{\text {cool }} / t_{\mathrm{ff}}$ is approximately constant with an average value of $t_{\text {cool }} / t_{\mathrm{ff}} \sim 30$ (blue line).
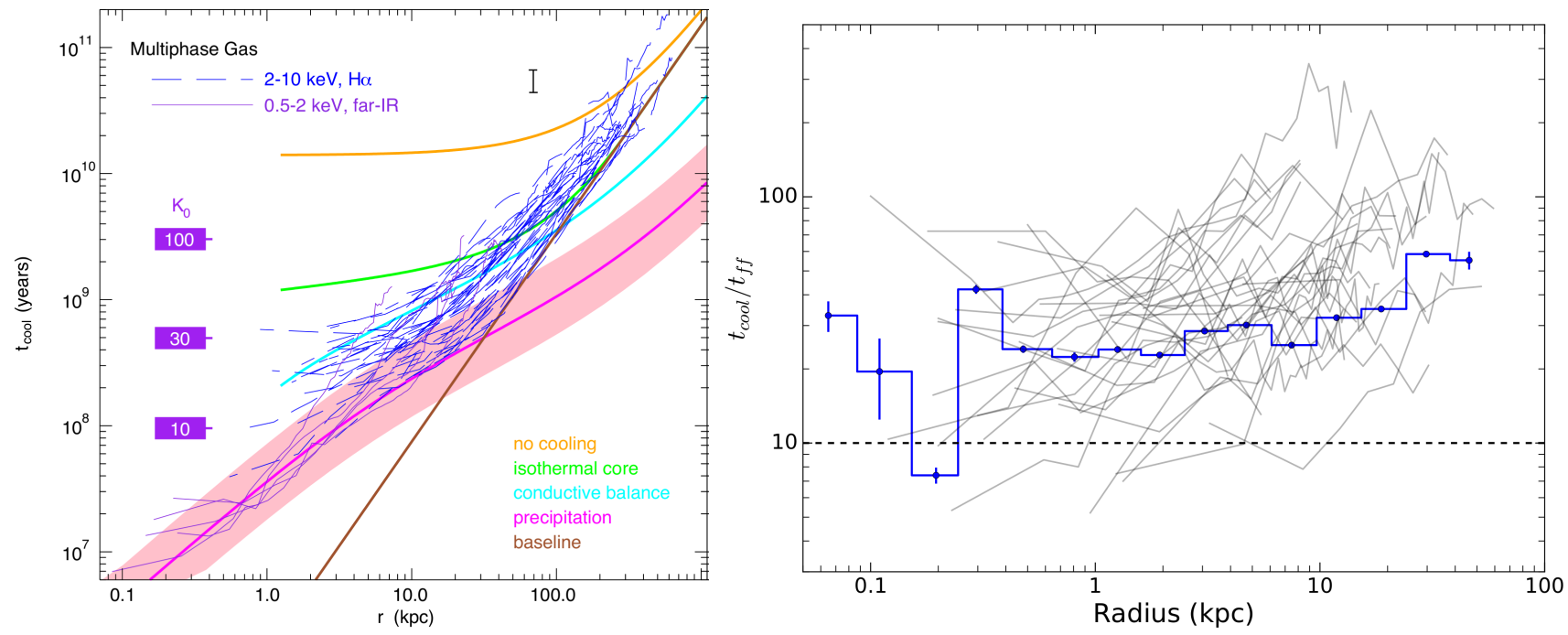

Figure 6. Observed timescales profiles in the IGrM (mainly related to TI). Left: Cooling time profiles for a sample of cool-core clusters (blue) and groups (magenta) hosting multiphase gas ( $\mathrm{H} \alpha, \mathrm{CO}$; figure reproduced from Voit et al. [159], arXiv author's version). The observed profiles are compared with the 'baseline' structure formation profile (maroon) and the typical simulation threshold $t_{\mathrm{cool}} / t_{\mathrm{ff}} \sim 10$ (pink). Right: The ratio of the cooling time to free-fall time in a sample of groups and ellipticals (the figure reproduced from Babyk et al. [155]; the average is shown with a blue line).

On the one hand, the above results show that the TI-ratio is correlated with the presence of multiphase gas. On the other hand, it is clear that the threshold is puzzlingly not unity (as expected in classical thermal instability), and that there is a substantial intrinsic scatter, even in multiphase systems. However, classical thermal instability starts from idealized linear fluctuations and it does not account for key astrophysical processes, such as AGN feedback or mergers, both recurrently injecting substantial gas motions (e.g., turbulence) at small and large radii, respectively (e.g., Lau et al. [164]; see Section 4.2). In 
this more realistic IGrM case of 'turbulent' nonlinear TI, the key physical timescale is not the free-fall time, but rather the turbulence eddy turn-over time (Gaspari et al. [165]),

$$
t_{\text {eddy }}=\frac{2 \pi R^{2 / 3} L^{1 / 3}}{\sigma_{v, L}}
$$

where $\sigma_{v}$ is the turbulence velocity dispersion and $L$ is the related injection scale. The velocity dispersion of the ensemble warm $\mathrm{H} \alpha$-emitting gas should linearly correlate with $\sigma_{v}$ of the hot IGrM, allowing for us to convert between the two, in particular by leveraging the higher spectral resolution of optical/IR telescopes. Rough estimates of the injection scale can be also obtained via the size of the ensemble warm gas filaments/nebulae, or via the AGN cavity diameter. In the presence of a turbulent halo, thermal instability develops chaotically and non-linearly in a very rapid way whenever $t_{\text {cool }} / t_{\text {eddy }} \sim 1$ [166-168]. Future X-ray microcalorimeter missions, like XRISM and Athena (see Section 6), will allow us to measure the eddy time directly and test, in more depth, the above scenarios. In Section 4 , we discuss the related processes from a theoretical perspective.

\subsubsection{Baryon Content}

A key quantity for AGN feedback models in cosmological simulations is the total integrated baryon budget and its dependence on halo mass. While galaxy cluster halos are massive enough to retain all of their baryons (e.g., [169]), energy injection by AGN feedback can lead to an overall depletion of baryons all the way out to the virial radius. Observational studies have found that the gas fraction within $R_{500}$ increases with halo mass [170-177]. Because an estimate of the gas density can be obtained from imaging data only, a lot of attention has been devoted to the study of gas density profiles $[175,178,179]$. Using a compilation of measurements from the literature, Sun [178] showed that, while the gas density of galaxy group cores is systematically lower than that of more massive systems, at $R_{500}$, the gas density is nearly independent of mass. Eckert et al. [175] studied the gas density profiles of the 100 brightest galaxy clusters and groups in the XMM-XXL survey. While, at galaxy clusters scales, the measured profiles show a well-defined core and a relatively steep decline in the outskirts, the density profiles of the selected groups exhibit a power-law behavior with a very flat index $n_{e}(r) \propto r^{-1.2}$, indicating that the gas fraction in spherical shells increases steeply with radius. It is believed that most of the gas has been evacuated from the inner regions under the influence of AGN feedback and displaced to larger radii, thereby explaining the observed shallow slopes [12,156].

In Figure 7, we present a compilation of published measurements of the hot gas fraction at $R_{500}$ as a function of the corresponding halo mass. The gas fractions were derived from $X$-ray data under the assumption of hydrostatic equilibrium, with the exception of XMM-XXL and SPT-SZ. In the case of XMM-XXL [175], weak lensing measurements for a sub-sample of 35 clusters were used to calibrate the mass-temperature relation. SPT-SZ masses [180] were derived as an ensemble from a joint fit to the cosmological parameters and the relation between SZ observable and halo mass. All of the studies find that the hot gas fraction contained within $R_{500}$ increases with halo mass roughly as $f_{\text {gas }} \propto M_{500}^{0.2}$. Here, we provide a conservative estimate of the $f_{\text {gas }}-M_{500}$ relation, which aims at encompassing all state-of-the-art observational studies and their uncertainties. To this aim, we collected the compilation of observational studies from Figure 7 and estimated in each mass bin the median and $90 \%$ confidence range of the data points. The resulting relation is shown as the gray band depicted in Figure 7. The gray band can be approximated as a power law, which reads

$$
f_{\text {gas }, 500}=0.079_{-0.025}^{+0.026} \times\left(\frac{M_{500}}{10^{14} M_{\odot}}\right)^{0.22_{-0.04}^{+0.06}} .
$$




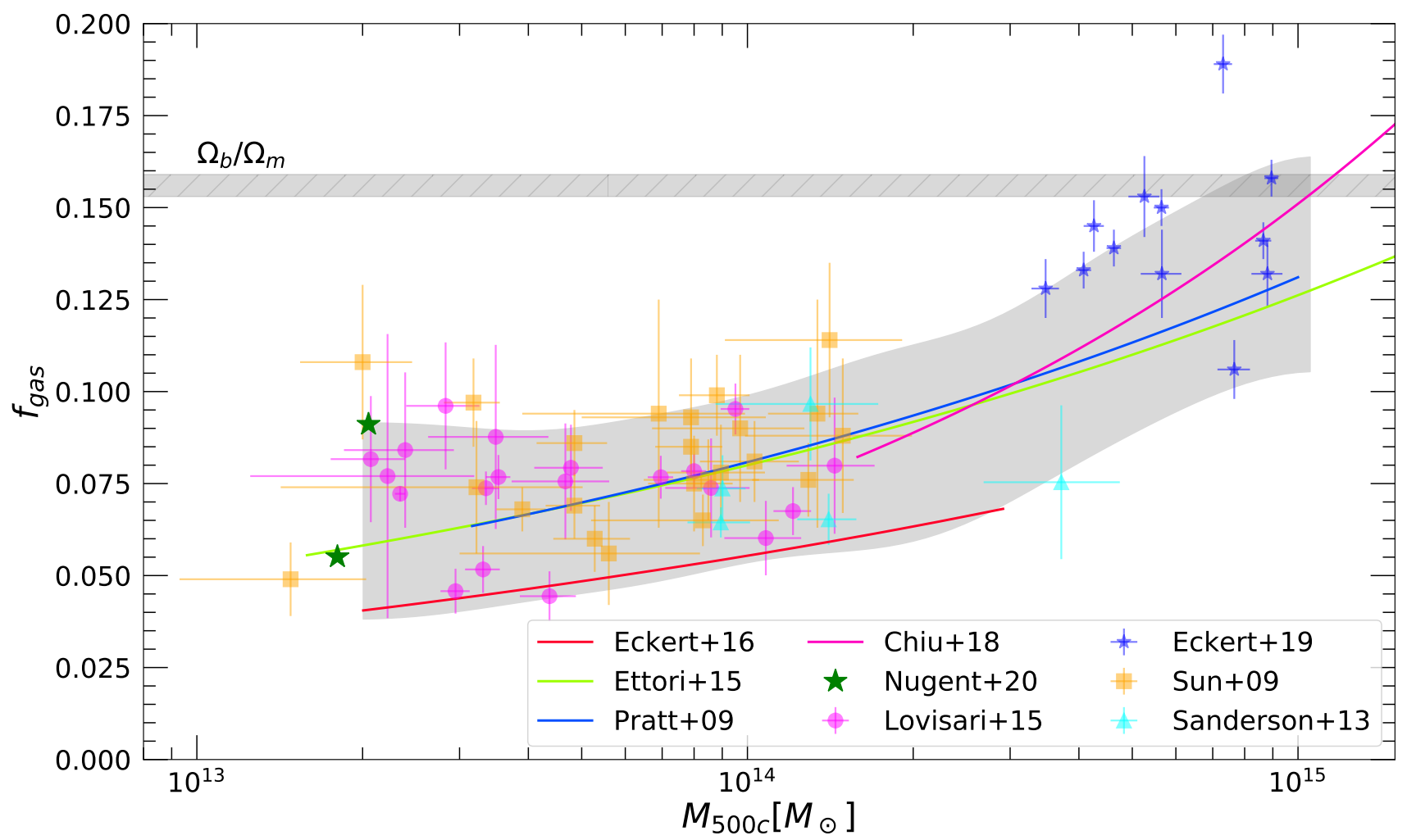

Figure 7. Compilation of existing measurements of the hot gas fraction at $R_{500}$ in galaxy groups and clusters as a function of halo mass $M_{500}$. The data points show the galaxy group samples of Sun et al. [137] (orange), Lovisari et al. [174] (magenta), Sanderson et al. [181] (cyan), and Nugent et al. [177] (green). The data from the X-COP sample [169] at the high-mass end are shown as the blue points for comparison. The solid lines show the $f_{g a s}-M$ relations that are derived from REXCESS (blue, [173]), XMM-XXL (red, [175]), SPT-SZ (magenta, [180]), and the literature sample of Ettori [176]. The gray shaded area shows the $90 \%$ confidence range encompassing the existing observational data and their corresponding uncertainties (see text).

While at the high-mass end, the gas fractions approach the cosmic baryon fraction, on galaxy group scales, the IGrM only contains about half of the baryons that are expected from the self-similar structure formation scenario. On the other hand, the stellar fraction $f_{\star}$ is a weak function of halo mass and decreases only slightly from $2-3 \%$ at $10^{13} M_{\odot}$ to $1-1.5 \%$ at $10^{15} M_{\odot}[5,6,172,175,180,182,183]$. The weak dependence of the stellar fraction on halo mass is insufficient to compensate for the steeper dependence of the gas fraction, which results in a deficit of baryons in galaxy groups with respect to the cosmic baryon fraction. Here, we note that this result is independent of the hydrostatic equilibrium assumption adopted by most authors. Indeed, an additional non-thermal pressure term would lead to a slight underestimation of the mass in these studies (e.g., [184]), which, in turn, would result in the gas fraction being overestimated [169]. Thus, a high level of non-thermal pressure would render the lack of baryons in group-scale halos even more severe.

Here, we caution that the measurement of the gas fraction of group-scale halos is a difficult one and it is hampered by numerous systematic uncertainties. While halo mass estimates definitely represent the leading source of systematics, several other sources introduce potential systematic errors. In the temperature range of galaxy groups, line cooling renders the $\mathrm{X}$-ray emissivity highly dependent on gas metallicity, which is difficult to measure away from group cores (see the review conducted by Gastaldello et al. within this issue). This can introduce uncertainties as large as $20 \%$ in the recovered gas mass [174]. Sample selection, usually based on ROSAT all-sky survey data, may bias the selected samples towards gas-rich systems, especially if the scatter at fixed mass is substantial $[185,186]$. Finally, most of the studies do not detect the X-ray emission all the way out to $R_{500}$ (see 
e.g., Figure 8 of [137]) and must rely on extrapolation. For all of these reasons, the question of what is the exact baryon fraction of galaxy groups within $R_{500}$ is still very much an open one, let alone within the virial radius.

\subsection{Radio Observations}

\subsubsection{Interaction between Radio Sources and the IGrM}

Radio surveys have made clear that the centers of galaxy groups and clusters are special locations for AGN (e.g., [89,187,188]), with group-central galaxies twice as likely to host radio-mode activity than non-central galaxies of equal mass out to $z>1$. Deeper observations show that almost all the central galaxies of $X$-ray luminous groups host some radio emission $[189,190]$, though in the local universe some of these may be contaminated by emission from low-level star formation [191]. The observations of nearby groups show a wide range of radio morphologies (e.g., [192]), with jet-mode feedback dominated by FR-I radio galaxies, as in clusters. Roughly one-third of X-ray luminous groups appear to host currently or recently active jet sources in their central galaxies [106] with typical jet powers in the range $10^{41}-10^{44} \mathrm{erg} \mathrm{s}^{-1}$ [190].

While cavities and shocks are the most accurate indicators of the impact of AGN feedback on the IGrM (see Section 3.1), current X-ray instruments have a limited ability to detect these features outside the high surface brightness cores of nearby groups. Radio studies offer an observationally cheaper way to measure feedback, particularly at higher redshifts. Radio galaxies are only periodically active and, once their AGN ceases to power them, their emission fades fastest at high frequencies. Therefore, low-frequency observations can be particularly effective at identifying older, dying radio sources, and measuring their full extent and luminosity (e.g., $[193,194])$. The radio spectrum can also give an indication of the properties of the source, most notably its age and the lobe pressure, which, for older sources, is usually in equilibrium with the surrounding IGrM. Combining radio and $\mathrm{X}$-ray observations, we can observe multiple cycles of outbursts in individual groups, e.g., NGC 5813 and NGC 5044 (Figures 2 and 8, [14,195]). In particular, in Figure 8, we show the existing high-quality radio, X-ray, and $\mathrm{H} \alpha$ observations of NGC 5044 [195]. GMRT $235 \mathrm{MHz}$ radio observations trace the oldest outburst via detached lobes and a bent, one-sided radio jet, while Chandra detects cavities on $\sim 5 \mathrm{kpc}$ and $\sim 150 \mathrm{pc}$ scales. Interestingly, the current radio jets, which are traced by high-resolution VLBA observations (bottom-left panel), are not aligned with the X-ray cavities, possibly indicating the precession of the jet axis with time.

The properties of group-central radio galaxies are closely linked to the IGrM. Both groups and clusters show a correlation between X-ray luminosity and the radio luminosity of the central source $[189,196,197]$. In clusters, central radio source luminosity is observed to be higher in systems with cooling times $<10^{9} \mathrm{yr}$ [198] and, in groups, it appears that radio jets are more common in the central galaxies of groups with short central cooling times, low $t_{\text {cool }} / t_{f f}$ ratios, and declining central temperature profiles [106]. However, perhaps the most important correlation is that between jet power, as determined from the enthalpy of AGN-inflated cavities, and radio luminosity. This $\mathrm{P}_{\text {cav }}-\mathrm{L}_{\text {radio }}$ relation was first established for galaxy clusters by Bîrzan et al. [92,112] and later extended to early-type galaxies [104] and galaxy groups [199]. Although there is significant scatter in the relation, it offers a mechanism for determining the energy that is available from AGN feedback in the many systems where direct determination in the X-ray is impossible.

Applying the $\mathrm{P}_{\text {cav }}-\mathrm{L}_{\text {radio }}$ relation to a large sample of SDSS groups and clusters with radio sources identified from the NVSS and FIRST surveys, Best et al. [89] showed that central radio galaxies dominate the heating of the IGrM within the cooling radius. They also found that the efficiency of AGN heating cannot be constant across the full mass range of groups and clusters; feedback must be less efficient in groups if they are not to be over-heated. Support for this result came from a study of groupsobserved in the COSMOS survey [200], which found that, factoring in the likely duty cycle of the AGN population, group-central radio galaxies can inject energies that are comparable to the binding energy 
of the IGrM. These results suggest that group-central AGN have the potential to drive gas out of the group core, and perhaps out of the group altogether, unless some mechanism reduces their effectiveness in heating the gas.

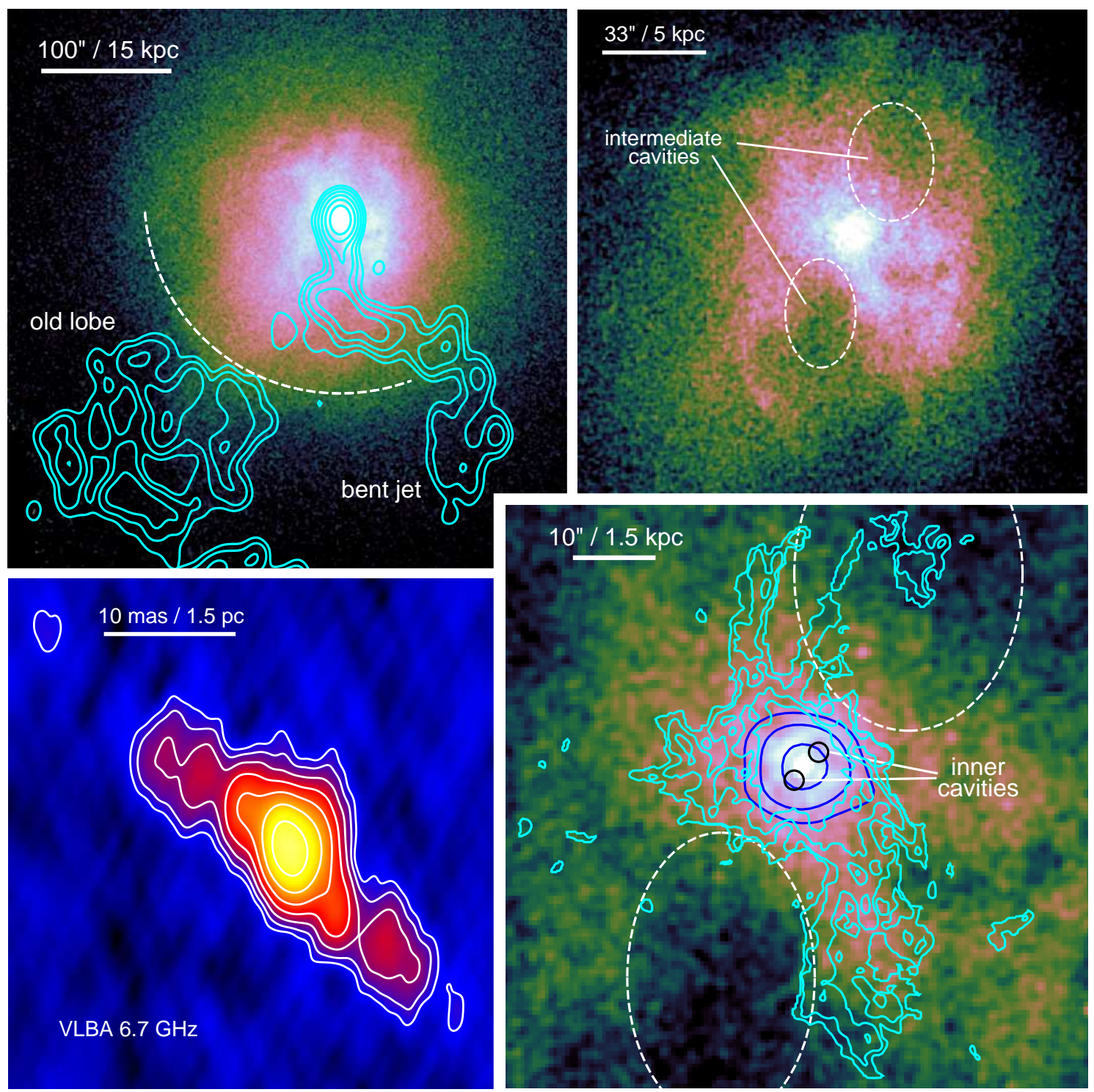

Figure 8. Multiple cycles of AGN feedback in the NGC 5044 galaxy group. The upper left panel shows the 0.5-2 keV Chandra image with GMRT $235 \mathrm{MHz}$ contours overlaid. These reveal an old, bent radio jet and detached lobe from a prior AGN outburst, whose structure has been affected by the sloshing front marked with a dashed line. The upper right panel zooms in to show more detail of the complex of cavities and cool filaments in the group core. The lower right panel zooms in further, with contours showing the MUSE H $\alpha$ (cyan) and ACA diffuse CO (blue) in the densest parts of the X-ray filaments and core. The lower left panel shows parsec-scale VLBA 6.7 GHz radio emission in the nucleus of the galaxy, evidence of a new cycle of AGN jet activity (adapted from [195]).

\subsubsection{Giant Radio Galaxies}

Because feedback studies at the group scale are largely limited to the X-ray bright cores of nearby groups where cavities are most easily identified, they have tended to focus on relatively small radio sources, with jet sizes of less than a few tens of kiloparsecs. However, the population of group-central radio galaxies includes much larger objects, some of which extend to very large radii, well beyond the cool core, and even into the outskirts of their groups. Pasini et al. [197] show that radio galaxies larger than $200 \mathrm{kpc}$ are more common in groups than clusters, and the largest radio galaxies are located in groups, probably because 
the IGrM is less able to confine their growth than the ICM. There is also evidence from new radio surveys, with greater sensitivity to extended diffuse emission, that giant radio galaxies may be more common, in general, than previously believed [201].

Large radio sources pose a problem for AGN feedback models, in that they may inject a large fraction of their energy into the IGrM at large radii, rather than in the core, where it is needed to balance cooling. Even some medium sized sources appear to have jets that tunnel out of the cool core and inflate cavities outside it (e.g., NGC 4261 [202]). IC 4296 is an extreme example in which at least one cavity is confirmed, which hosts an FR-I radio galaxy whose $160 \mathrm{kpc}$ diameter lobes extend out to a projected radius of $\sim 230 \mathrm{kpc}$ [203]. This is far beyond the cool core (20-30 kpc radius) and about half of $R_{500}$ for this $\sim 1 \mathrm{keV}$ group. In such a system, while some of the energy that is involved in lobe inflation will likely have heated the core, the energy that is bound up in the relativistic particles and magnetic field of the radio lobes will likely be released at large radii, heating gas that is unlikely to contribute to fuelling the AGN. Other nearby examples of group-central giant FR-Is include NGC 315 and NGC 383 [192] and NGC 6251 [204]. As in clusters, group-central FR-II galaxies are uncommon, but not unknown (see, e.g., [196,205]). Their faster, more collimated jets likely provide feedback heating via shocks during expansion (as in, e.g., $3 C$ 88, [115]), and lobe inflation will drive turbulence, but, as with the giant FR-Is, it is less clear how they affect the cooling region once they grow beyond it.

Therefore, giant radio galaxies pose a number of important questions for feedback models of groups. Do they provide feedback that can balance the rapid cooling in group cores, and if so how? The large sizes of these systems, particularly the FR-Is, implies that their jets have been active for very long periods. How do these sources stay active for so long?

\subsection{Multiwavelength Observations}

In the cool cores of galaxy clusters, many observations have shown evidence of material cooling from the hot atmosphere, in the form of highly multi-phase filamentary nebulae surrounding the central galaxy and containing gas and dust with temperatures ranging from $\sim 10^{6} \mathrm{~K}$ to a few $\times 10 \mathrm{~K}$. Some cool core galaxy groups show similar structures, although they are generally less luminous and are thus far less thoroughly explored. As of yet, few studies have specifically focused on BGGs, but samples of giant ellipticals provide a window on the group regime.

$\mathrm{H} \alpha$ emission from ionized gas with temperatures $\sim 10^{4} \mathrm{~K}$ may be the most accessible tracer of cooled material. Lakhchaura et al. [206] find that, in giant ellipticals, as in galaxy clusters (c.f. [207]), the presence of $\mathrm{H} \alpha$ emission is associated with high IGrM densities, short cooling times, low values of the thermal instability criterion, $t_{\text {cool }} / t_{\mathrm{ff}}$ (see Section 3.1.3), and disturbed $\mathrm{X}$-ray morphologies, with the overlap between galaxies with and without detected $\mathrm{H} \alpha$, suggesting that the transition between the two states can happen fairly easily. They also report a weak correlation between the mass of $\mathrm{H} \alpha$-emitting gas and $\mathrm{P}_{\mathrm{cav}}$, as expected if the $\mathrm{H} \alpha$ traces cooling material, some of which will eventually fuel the AGN. Some of the best known X-ray bright groups contain examples of $\mathrm{H} \alpha$ filaments that are similar to those seen in clusters (e.g., [14,208-210]). As in clusters, the filaments are closely correlated with feedback-related structures, showing signs of having been drawn out behind, or wrapping around, radio lobes and cavities. In some cases they are located in cool X-ray filaments that show signs of being thermally unstable [209]. Figure 8 shows an example of this in the NGC 5044 group, where the $\mathrm{H} \alpha$ nebula is correlated with the brightest cool X-ray emission and it appears to wrap around the base of the intermediatescale cavities. Spatially resolved spectroscopy shows that, while the inner parts of these $\mathrm{H} \alpha$ nebulae are generally cospatial with the stellar bodies of the BGGs, they do not rotate with the stars, supporting formation from the IGrM rather than stellar mass loss (e.g., [211-213]). It should be noted that, while BGGs do host some star formation (SF), their $\mathrm{H} \alpha$ nebulae are not tracing SF. McDonald et al. [214] studied the relation between the star formation rate inferred from infrared data and the X-ray cooling luminosity (Section 3.1) and found 
that the inferred star formation rates in BGGs are typically quenched by a factor 10-100 as compared to the pure cooling scenario.

Molecular gas in groups has been observed via multiple tracers. Herschel observations revealed [CII] emission from $\sim 100 \mathrm{~K}$ gas with a similar distribution to the $\mathrm{H} \alpha$, and [CII]/H $\alpha$ flux ratios indicating that both phases are powered by the same source [208]. Spitzer IRS spectra show rotational $\mathrm{H}_{2}$ lines in the BGGs of some X-ray bright groups [215], tracing gas at a few $\times 100 \mathrm{~K}$, and $\mathrm{CN}$ has been detected in absorption in a handful of cases via the millimeter-wave band [216]. The forthcoming James Webb Space Telescope will open an important observation window on $\mathrm{H}_{2}$, which is likely the dominant mass component of the molecular phase. However, at present, emission from $\mathrm{CO}$ is our best tracer for this phase, allowing for us to examine the coolest, densest gas in the cooling regions of groups.

Babyk et al. [217] examine CO in a large sample of local ellipticals (many of which are BGGs) and find that the molecular gas mass $\mathrm{M}_{m o l}$ is correlated with the density of the IGrM and its mass in the central $10 \mathrm{kpc}$, and that systems with $\mathrm{t}_{\text {cool }}<1 \mathrm{Gyr}$ at $10 \mathrm{kpc}$ are more likely to contain molecular gas. They also find that $\mathrm{M}_{\text {mol }}$ is proportional to $\mathrm{P}_{\text {cav }}$, confirming that the molecular gas is the fuel source for the central AGN. However, cooling from a surrounding hot halo is not the only source of gas for ellipticals. Davis et al. [218] use a combination of the ATLAS3D and MASSIVE samples to show that gas-rich mergers are an important source of molecular gas in these galaxies. The observations of smaller samples of BGGs find some of the same trends, and show that BGGs of X-ray bright, cool core groups are not the CO-richest systems $[219,220]$. BGGs of X-ray fainter groups can contain more $\mathrm{CO}$ (and $\mathrm{HI}$ ), and it is more often located in disks, rather than filaments. This, again, emphasizes the importance of gas-rich mergers in groups, although IGrM cooling is likely still the more important process in the cool core groups in which AGN feedback is most often observed.

The BGGs of X-ray bright cool core groups generally seem to contain only a few $\times 10^{6}$ or $\times 10^{7} \mathrm{M}_{\odot}$ of molecular gas [220], which makes them challenging targets, even for ALMA. However, being nearer than typical cool core clusters, groups offer an opportunity to study individual molecular cloud associations within the cool core, rather than the overall filamentary structures. Three well-known systems have been studied in detail by ALMA, NGC 4636, NGC 5846 [221], and NGC 5044 [209,222]. The velocity dispersions of the molecular clumps that were observed in these systems suggest that they are not gravitationally bound, and they are likely collections of smaller, denser clouds, with more diffuse gas between them. Atacama Compact Array (ACA) observations of NGC 5044 show that a significant fraction of the molecular gas in the BGG is more diffuse than the clumps observed by ALMA [213], and a similar argument can be made for the other two groups by comparing the CO masses that are derived from ALMA and IRAM 30m observations. The denser CO clumps are generally located within filamentary structures visible at other wavelengths [221], and the extent and velocity distribution of the diffuse CO in NGC 5044 is similar to that of the $\mathrm{H} \alpha$ and [CII] emission, supporting the idea that all of the observed phases are material cooling from the IGrM. Figure 8 shows the diffuse $\mathrm{CO}$ emission in the group core, collocated with the peak of the $\mathrm{H} \alpha$ and X-ray emission. As with $\mathrm{H} \alpha$, the $\mathrm{CO}$ in these ALMA-observed systems is cospatial with the stellar component, but shows little sign of rotation or velocity gradients, consistent with formation from the IGrM.

Intriguingly, ALMA studies of giant radio galaxies, some of them group-central systems, show a different $\mathrm{CO}$ morphology, with the molecular gas being located in compact disks $[223,224]$. The difference in cold gas morphology may indicate a difference in the fuelling of the AGN. There are examples of group-dominant giant radio galaxies that appear to be fed by cold gas (e.g., NGC 1167, [225]) and, given the importance of galaxy interactions in groups, the potential for fuelling by gas rich mergers cannot be ignored. With only a handful of group-dominant galaxies mapped thus far in molecular gas, there is a significant opportunity for the exploration of the mechanics of AGN fuelling in these important systems. 


\section{Theoretical Framework}

Hot halos are a fascinating and crucial element of virialized systems in the Universe, which have been unveiled to be a fundamental engine for the growth and triggering of $\mathrm{SMBH}$, despite the large difference in spatial and temporal scales, which span over nine orders of magnitude (commonly sub-divided in three major scales; see Figure 9: micromeso-macro). Here, we review the AGN feedback process in terms of fundamental physics and why it is expected in the more theoretical framework of accretion out of the hot halo and onto $\mathrm{SMBH}$, with a keen eye on galaxy groups. It is important to appreciate that AGN feedback is only half of the self-regulated cycle, which is bootstrapped via the AGN feeding, the key complementary mechanism on which we will also focus below, as shown in the summary diagram of Figure 9.

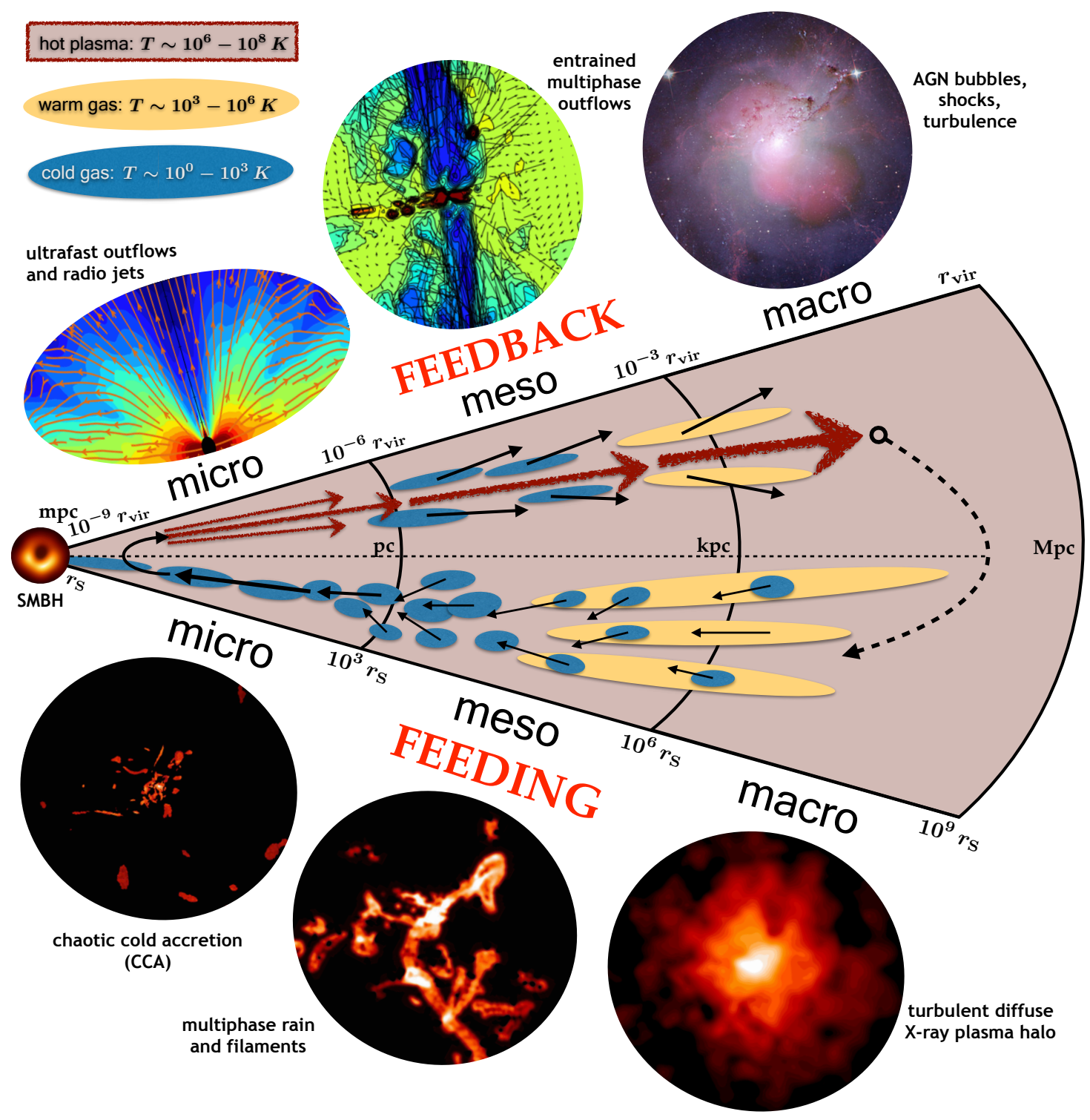

Figure 9. A schematic representation of the self-regulation loop necessary to fully link feeding and feedback processes over nine orders of magnitude in space (and time) and over the multiphase/multiband cascade, including Xrays (hot halo/outflows), the IR/optical (warm filaments), and radio/mm (molecular clouds) bands-reproduced from Gaspari et al. [11] (arXiv authors' version). In particular, the IGrM experiences relatively stronger top-down condensation rain compared with clusters, due to the lower central cooling times. Tightly related to such an enhanced feeding is the higher frequency of the AGN outflow/jet feedback events, which gently self-regulate each galaxy group for several billion years during the cosmic evolution. 
While complex, non-linear thermo-hydrodynamical (THD) mechanisms are at play over the macro (kpc-Mpc), meso (pc-kpc), and micro scales (mpc-pc) - thus requiring expensive numerical 3D Eulerian simulations-it is useful here to understand the whole $\mathrm{SMBH}-$ halo system as a unified, co-evolving engine. In essence, such a global THD system can be described via the simple conservation of energy, or analogously via the (Lagrangian) entropy equation (Peterson and Fabian [83], Gaspari [226]):

$$
U \frac{d}{d t} \ln K=\mathcal{H}-\mathcal{L},
$$

where $K=k_{\mathrm{b}} T / n^{\gamma-1}$ is the astrophysical entropy (with $n=n_{\mathrm{e}}+n_{\mathrm{i}} \approx 2 n_{\mathrm{e}}$ the sum of the electron and ion number densities), $U=P /(\gamma-1)$ is the internal/thermal gas energy per unit volume $(\gamma=5 / 3$ is the IGrM adiabatic index $), \mathcal{H}$ and $\mathcal{L}$ are the gas heating and cooling rates per unit volume ( $\mathrm{erg} \mathrm{s}^{-1} \mathrm{~cm}^{-3}$ ), respectively. A few immediate insights from Equation (12): the internal energy acts as an effective normalization knob (the larger the X-ray temperature times density, the stronger the required heating/AGN feedback, in absolute erg s ${ }^{-1}$ values); secondly, the macro entropy evolution is the sole result of the competition of heating and cooling processes, which translates in the competition between AGN feedback and feeding. Let us first discuss the (astro)physics and consequences of the cooling/feeding component of the cycle, i.e., $\mathcal{L}$.

\subsection{AGN Feeding E Cooling Processes}

The cooling process is very well understood from basic quantum physics and laboratory plasma/ionized gas experiments, with a radiative cooling loss [227] $\mathcal{L}=n_{\mathrm{e}} n_{\mathrm{i}} \Lambda(T, Z)$, where $\Lambda$ is a cooling function varying with gas temperature and metallicity $Z\left(\sim 0.6-1 Z_{\odot}\right.$ for group cores; Mernier et al. [228], cf. the companion Gastaldello et al. review). The hot IGrM experiences a significantly enhanced $\Lambda$ due to the influence of line cooling (mostly recombination) taking over from the Bremsstrahlung/free-free emission $\left(\Lambda \propto T^{1 / 2}\right)$, which instead shapes the more massive galaxy clusters $(T>2 \mathrm{keV})$, as shown in Figure 10.

Figure 10 depicts the three main (quasi)stable phases that arise during the top-down condensation cascade [166], especially during the feeding dominated stage of the AGN cycle. Assuming relatively slow motions over the group macro gravitational potential (quasi pressure equilibrium), Equation (12) can be approximated as (e.g., Pope [229])

$$
H-L \approx \frac{c_{\mathrm{s}}^{2}}{\gamma-1} \dot{M}_{\text {net }},
$$

where $c_{\mathrm{s}}^{2}=\gamma k_{\mathrm{b}} T / \mu m_{\mathrm{p}}$ is the (squared) IGrM sound speed (with $\mu \approx 0.62$ the mean atomic weight of the IGrM) and $H / L$ is the gas heating/cooling power (or luminosity in erg s ${ }^{-1}$ ). Notably, any net cooling or heating will induce a net mass inflow $\left(\dot{M}_{\text {cool }}\right)$ or outflow rate $\left(\dot{M}_{\text {OUT }}\right)$ in the macro-scale halo, being denoted as $\dot{M}_{\text {net }} ;$ here, uppercase subscripts denote macro properties, while lowercase subscripts denote micro properties. Therefore, the thermal evolution of the IGrM is deeply intertwined with feeding/feedback processes, as intuitively anticipated above. 


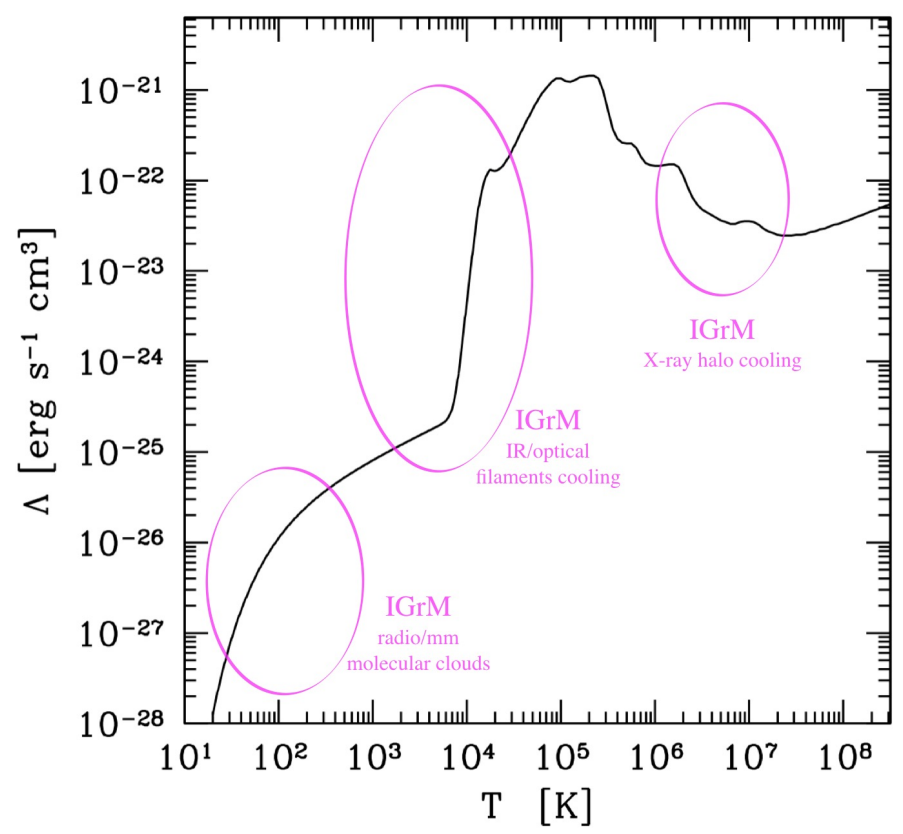

Figure 10. Multiwavelength cooling function for the IGrM (adapted from Gaspari et al. [166], unifying the atomic/plasma physics studies by Sutherland and Dopita [227], Dalgarno and McCray [230], Inoue and Inutsuka [231]; $\left.Z=1 Z_{\odot}\right)$, which was specifically used for a typical galaxy group akin to NGC 5044. Above the neutral hydrogen recombination $\left(T \sim 10^{4} \mathrm{~K}\right)$, the gas is fully ionized and in collisional ionization equilibrium; below this threshold, the gas becomes progressively less ionized ( $\lesssim 1 \%$ ), leading to the formation of neutral filaments and, subsequently, dense molecular clouds. The three magenta ellipses highlight the three key (semi)stable phases of the condensing IGrM, in particular during the feeding dominated part of the AGN cycle that is shown in Figure 9 (bottom insets).

In the absence of any heating, the entire hot atmosphere would rapidly condense and collapse, initiating from the inner denser radial regions (see Section 3.1). As noted in the previous sections, such massive cooling flows are not observed in our Universe, especially in galaxy groups. On the other extreme of (idealized) feeding models, the IGrM halo might experience pure cooling while having significant angular momentum. In this regime, the gas would condense through helical paths onto the equatorial plane, and there form a thin rotating multiphase disk [166]. While extended disks have been found in some BGGs (Hamer et al. [232], Juráňová et al. [233], Ruffa et al. [223]), such a scenario would induce both large (unobserved) cooling rates in X-ray spectra, as well as drastically reduced accretion rates onto the SMBH due to the preservation of high angular momentum and related centrifugal barrier.

Realistic IGrM atmospheres, instead, often reside in an intermediate THD regime, neither strongly rotating nor in a spherical cooling flow (David et al. [209], Lakhchaura et al. [206], O'Sullivan et al. [106], Temi et al. [221]—Section 3.3). Indeed, hot halos experience significant amount of turbulence, with an irreducible level of 3D turbulent velocity dispersion $\sigma_{v} \approx 100$ $300 \mathrm{~km} \mathrm{~s}^{-1}$, due to both the previous AGN feedback outbursts and the secular cosmological flows (e.g., Vazza et al. [234], Valentini and Brighenti [235]), as shown by high-resolution HD/cosmological simulations (Lau et al. [164], Gaspari et al. [236], Hillel and Soker [237], Weinberger et al. [238], Wittor and Gaspari [239]) and X-ray spectroscopy (Sanders and Fabian [240], Ogorzalek et al. [241], Hitomi Collaboration et al. [242]). While we review the kinematical features in a companion review (Gastaldello et al.), here we focus on its thermodynamical impact, namely the formation of chaotic cold accretion (CCA) and related multiphase rain, a key process driving the bulk of AGN feeding and, hence, the recurrent AGN feedback triggering. In a turbulent hot halo, chaotic multiscale eddies drive local perturbations in relative gas density proportionally to the turbulence sonic Mach number 
$\left(\delta n / n \propto \mathcal{M}_{t} ;\right.$ Gaspari and Churazov [243], Zhuravleva et al. [244]). The relative increase in IGrM density produces in-situ enhanced radiative cooling $\left(\mathcal{L} \propto n^{2}\right)$, thus leading to turbulent non-linear thermal instability (TI; Gaspari et al. [245], Voit [246]). It is important to note that such a chaotic instability is different from classical TI (Field [161], McCourt et al. [157], Pizzolato and Soker [247]), in the sense that direct non-linear fluctuations are seeded by chaotic motions, rather than growing from tiny linear amplitudes. This triggers a quick top-down condensation of localized (soft X-ray) patches to the first quasistable phase at $T \sim 10^{4} \mathrm{~K}$, which is best traced via ionized line-emitting (e.g., $\mathrm{H} \alpha+[\mathrm{NII}]$; Gastaldello et al. [13], McDonald et al. [248], Werner et al. [208]) filaments or nebulae observed in optical/UV (e.g., see the synthetic image in the bottom middle inset of Figure 9). Sustained turbulent perturbations lead to the further condensation cascade onto the last stable and compact gas phase, molecular gas clouds (bottom left inset of Figure 9; see Section 3.3). Such cold clouds will then strongly and frequently collide inelastically within the meso/micro scale, cancelling angular momentum and, thus, feeding the central SMBH (hence, the 'CCA' nomenclature; Gaspari et al. [166]), with the consequent trigger of the next stage of AGN feedback (Section 4.2). CCA feeding recurrently boosts the accretion rates over 100 fold over the feeble and quiescent hot-mode (Bondi [249], Narayan and Fabian [250]) accretion, thereby overcoming the inefficiency of classical hot mode accretion.

On the macro scale, the hot halo can be assessed to reside or predicted to soon enter the CCA raining phase, whenever the ratio of the plasma cooling time and the turbulence eddy gyration/turnover time reaches unity. This reference dimensionless number is called C-ratio (from condensation or CCA; Gaspari et al. [165], Olivares et al. [167]),

$$
C \equiv \frac{t_{\text {cool }}}{t_{\text {eddy }}} \sim 1,
$$

where the cooling and turbulence timescales have been defined in Equations (8) and (10). A correlated ratio and thermal-instability threshold is the TI-ratio $\equiv t_{\text {cool }} / t_{\text {ff }} \lesssim 10-30$ (e.g., Gaspari et al. [156], Sharma et al. [158], Voit et al. [251]), where the free-fall time is defined in Equation (9). As introduced in Section 3.1.3, all three IGrM timescales can be constrained from X-ray or optical/IR datasets. While both the $C$-ratios and TI-ratios are valuable complementary tools, the simulations show that the $C$-ratio is the more direct physical criterion to apply to probe the onset and extent of nonlinear thermal instability (e.g., Gaspari et al. [165]; see also Figure 17). Indeed, unlike in classical linear TI, turbulence acts as an irreducible background of fluctuations over the whole IGrM (e.g., Lau et al. [164]). In particular, AGN bubbles are a key recurrent mechanism for inducing such fluctuations in the IGrM (e.g., McNamara et al. [252], Voit [253]). In this regard, it is not surprising that $t_{\text {cool }} / t_{\mathrm{ff}}$ profiles show a large non-trivial deviation above unity, as well as a large intrinsic scatter (e.g., Singh et al. [254]; also see Figure 6). Figure 11 shows the average cooling and turbulence eddy time profiles (with scatter) for the galaxy group regime (Gaspari et al. [165]). Evidently, the crossing of $C \sim 1$ matches the dotted circle denoting the typical size of the condensed extended multiphase nebulae well (e.g., McDonald et al. [248]).

Together with mild C-ratios, a CCA-driven atmosphere-often found in the IGrM coresis described by a low turbulent Taylor number (Juráňová et al. [168], Gaspari et al. [255]):

$$
\mathrm{Ta}_{\mathrm{t}} \equiv \frac{v_{\mathrm{rot}}}{\sigma_{v}} \lesssim 1 .
$$

Given that the dominant galaxies of hot gas rich galaxy groups tend to be early-type (as do those galaxies that host their own extended hot halos), they have a fairly weak coherent gas rotational velocity $v_{\text {rot }}$ (e.g., Caon et al. [256], Diehl and Statler [257]), unlike lower-mass/spiral galaxies. Therefore, a median IGrM long-term evolution is to oscillate between stages of strong CCA rain $\left(\mathrm{Ta}_{\mathrm{t}} \sim 0.3-1, C \sim 0.5-1\right)$ and mild rain superposed on a clumpy disk $\left(\mathrm{Ta}_{\mathrm{t}} \sim 1-3, C \sim 1-2\right)$. Evidently, extremes of strong rotation $\left(\mathrm{Ta}_{\mathrm{t}} \gg 1\right)$ or overheated quiescence $(C \gg 1)$ can lead to periods of disk- or Bondi-driven accretion, both experiencing highly suppressed inflow rates and feedback (albeit such periods must 
be short-lived to avert the cooling flow catastrophe). High-resolution HD simulations have shown that the chaotic behaviour of a CCA-driven halo is imprinted not only in the thermodynamical maps and kinematical properties, but also in the time-series spectra. Unlike quiescent and continuous hot modes (Bondi/ADAF), CCA drives a characteristic flicker/'pink' noise power spectrum (logarithmic slope of -1) in the Fourier space of frequencies $f$ (Gaspari et al. [166]), thus generating strong self-similar variability on all temporal scales (the integral over $d f$ of this spectrum yields constant variance), from several Myr down to years and minutes, as ubiquitously observed in multiwavelength AGN lightcurves (Ulrich et al. [258], Peterson [259]). This triggers the second key part of the self-regulated loop, AGN feedback, the focus of the next section.

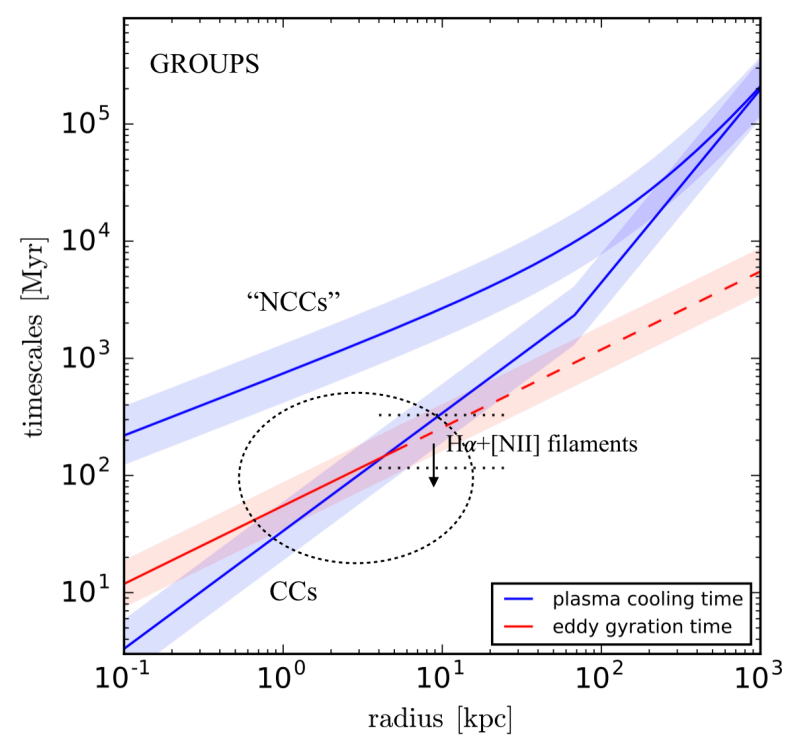

Figure 11. The average cooling time and turbulence eddy time profiles in the IGrM (with $90 \%$ confidence scatter bands), the latter constrained mainly via optical/IR telescopes (figure reproduced from Gaspari et al. [165]; group sub-sample). The dotted circle marks the size of the condensed warm nebular emission, which matches the $C \equiv t_{\text {cool }} / t_{\text {eddy }} \sim 1$ turbulent TI threshold.

\subsection{AGN Feedback \& Heating Processes}

While pure cooling flows and catastrophic condensation are not detected in IGrM observations, strong overheating is equally ruled out, as virtually all galaxy groups exhibit central cooling times well below $1 \mathrm{Gyr}$ due to the high efficiency of radiative cooling in their characteristic temperature range (see Figure 10). We note that massive galaxy clusters, instead, show a dominant population of non-cool-core systems with central cooling times above the Hubble time (e.g., [260,261]). The system would be in perfect thermal equilibrium in the absence of any heating and cooling terms, as inferred from Equation (12). However, an analogous configuration can be achieved if a heating process (macro AGN feedback) balances the cooling rate (macro condensation). This configuration is the more realistic state of observed galaxy groups, with the characteristic feature that the self-regulation process is intrinsically chaotic (from the macro down to micro scales; Section 4.1), hence only leading to a statistical thermal balance $\mathcal{H} \sim\langle\mathcal{L}\rangle$. Moreover, while Equation (12) formally allows for the entropy to decrease (pure cooling), fundamental THD physics dictates that entropy shall always increase in real systems (even over the ensemble Universe). With such intuition, we can already expect that the heating rate is as essential-if not eventually more vigorous-than the cooling component (Figure 9).

Before tackling the AGN feedback physical sub-processes, here we first discuss the key difference between groups and clusters. In Figure 12, we show an analysis of the potential impact of the stored SMBH energy versus the gravitational binding energy of the hot halo cores (from small groups to clusters), by leveraging the large sample of Gaspari et al. [77] 
with both direct $\mathrm{BH}$ masses and extended hot halos detected. The potentially available $\mathrm{SMBH}$ mechanical energy is $E_{\mathrm{BH}}=\varepsilon_{\mathrm{M}} M_{\mathrm{BH}} c^{2}$, where $\varepsilon_{\mathrm{M}}$ is the macro mechanical efficiency (Gaspari and Sądowski [262]). We test for the now commonly used fixed $\varepsilon_{\mathrm{M}} \sim 10^{-3}$ (we will explore variations to this basic modeling further below). The gravitational binding energy is tightly related to the thermal energy via the virial theorem, $E_{\mathrm{bind}} \approx 2 E_{\mathrm{th}} \propto M_{\mathrm{gas}} T_{\mathrm{x}}$. Here, we consider the integration over a large scale, $R<0.15 R_{500}$. Evidently, the linear regression fit (including the intrinsic scatter band) is significantly shallower than the dashed line of the one-to-one balance. In particular, the mechanical feedback energy that a SMBH can release could potentially overcome the core binding energy if released in a very short period of time-for instance, assuming a quasar-like/Sedov blast scenario. This would drastically overheat and evacuate the gaseous core atmosphere, becoming more serious toward the galaxy group regime and lower mass halos $\left(E_{\mathrm{bind}, \mathrm{c}}<10^{59} \mathrm{erg}\right)$, where the feedback energy might even evacuate the entire gas virial region (Puchwein et al. [22], Gaspari et al. [263], as in Section 5.2). Because observations almost ubiquitously detect hot atmospheres, this indicates that the AGN feedback in groups shall be well self-regulated and relatively gentler than in massive galaxy clusters, which can sustain much stronger and impulsive AGN feedback deviations over the cosmic evolution.

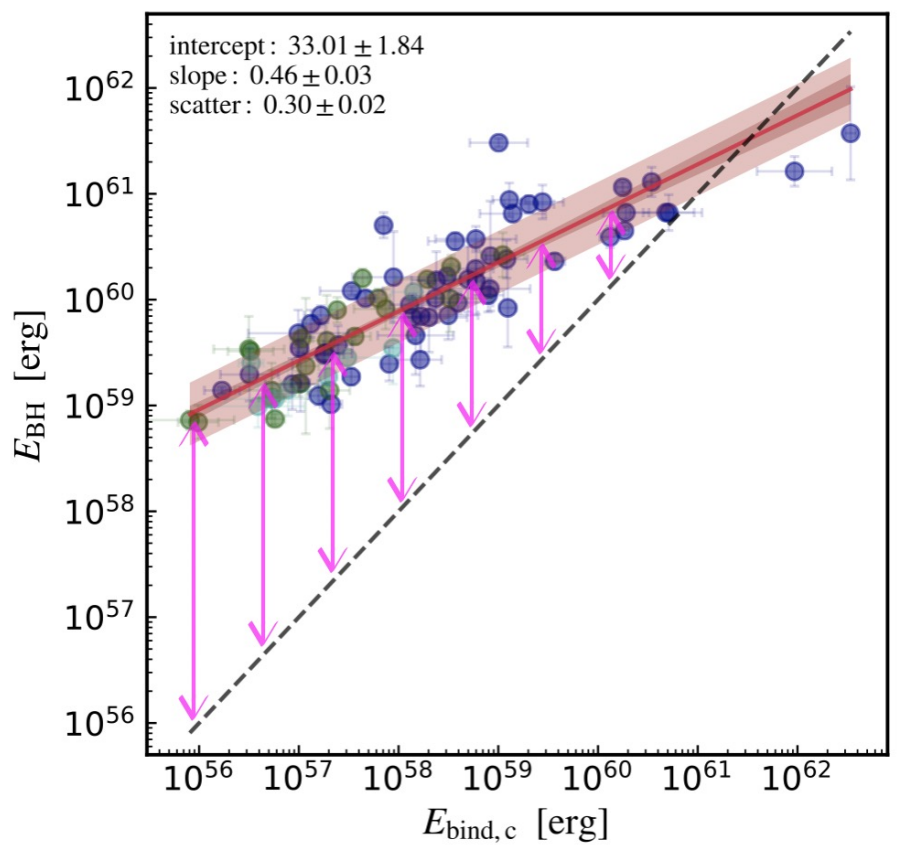

Figure 12. Available mechanical feedback energy of the central SMBH versus gravitational binding energy of the hot gas within the core of the host halo $\left(R \lesssim 0.15 R_{500}\right)$. The SMBH energy is $E_{\mathrm{BH}}=10^{-3} M_{\mathrm{BH}} c^{2}$, while the binding energy is related to the thermal energy via the virial theorem $E_{\text {bind }} \approx 2 E_{\text {th }} \propto M_{\text {gas }} T_{\mathrm{x}}$. The 85 points are taken from Gaspari et al. [77], which include the observed direct/dynamical SMBH mass with the X-ray halo detected in the host group or cluster. The solid red curve shows a fit to the relation with a power law, with the 16-84 percentile interval being indicated by the red shaded area. The 1- $\sigma$ intrinsic scatter is plotted as a light red band on top of the mean fit. The circle colors reflect the morphological type of the central galaxy: elliptical (blue), lenticular (green), and spiral (cyan). The black dashed line demarks the one-to-one energy equivalence, whereas the magenta arrows highlight the excess $\mathrm{BH}$ energy when compared to the binding energy.

Reaching a gentle self-regulation, while avoiding strong overheating, implies two major features of AGN feedback in galaxy groups and related improvements when compared with the above modeling. First, the conversion efficiency of accreted rest mass energy into feedback energy is expected to decline with lower halo mass: equating the macro AGN power to the gas $X$-ray radiative cooling rate requires to modify the above with 
$\varepsilon_{\mathrm{M}} \sim 10^{-3}\left(T_{\mathrm{x}} / 2 \mathrm{keV}\right)$ (Gaspari and Sądowski [262]; see also Equation (16)). This can be explained by the weaker macro-scale coupling of the AGN jets/outflows with the hot halo, as the IGrM atmospheres are more diffuse than the dense ICM counterparts. Second, in order to avoid the above evacuation outburst, such self-regulated AGN feedback has to be not only gentler, but significantly more frequent, i.e. with larger duty cycle (ratio of on/off activity). This is also naturally explained by the relatively lower cooling times (tens of Myr) in the inner IGrM regions (as compared with the ICM counterparts), due to the lower $T_{x}$ and the substantially enhanced $\Lambda$ via line emission (Figure 10). Both such key features have been extensively tested and retrieved by high-resolution HD simulations (Gaspari et al. [156], Sharma et al. [158], Gaspari et al. [264], Prasad et al. [265]), and found in observations (e.g., Best et al. [89], O'Sullivan et al. [106]).

While we have discussed the key characteristics and requirements of AGN heating, the next major question is: how is the AGN feedback energy propagated and dissipated within the IGrM? The problem is challenging, as it entails a wide range of scales and phases, from the milliparsec up to at least the $100 \mathrm{kpc}$ region, as shown in Figure 9 (top insets). Generalrelativistic, radiative-magnetohydrodynamical simulations (GR-rMHD; Sądowski and Gaspari [266]) resolving radial distance of $\sim 500 r_{S}$ (Schwarzschild radii) show that the AGN triggered via CCA is able to transform the inner gravitational energy into wide ultrafast outflows (UFOs) with velocities $\sim 0.1 c$ (top-left inset in Figure 9; Fukumura et al. [267], Tombesi et al. [268]). Under strong magnetic field tower and spin conditions (Tchekhovskoy et al. [269]), the AGN is also able to generate a very collimated relativistic (radio-emitting) jet, perpendicularly to the thick accretion torus. The above GR-rMHD simulations show that kinetic feedback appears to be present over both low and high Eddington ratios $\left(\dot{M}_{\mathrm{BH}} / \dot{M}_{\mathrm{Edd}} \equiv \dot{M}_{\mathrm{BH}} /\left[23 \mathrm{M}_{\odot} \mathrm{yr}^{-1}\left(\mathrm{M}_{\mathrm{BH}} / 10^{9} \mathrm{M}_{\odot}\right)\right]\right)$, with a retrieved micro mechanical efficiency $\varepsilon_{\mathrm{m}} \simeq 0.03 \pm 0.01$. At variance, the radiative efficiency declines dramatically below $\varepsilon_{\mathrm{r}} \ll 0.01$ at $\dot{M}_{\mathrm{BH}} / \dot{M}_{\mathrm{Edd}}<1 \%$, which is the typical regime of local AGN in massive galaxies (Russell et al. [270]). Further, in order to achieve an efficacious macro self-regulation, the AGN feedback has to satisfy energy conservation (Costa et al. [271]) and related micro- to macro-scale power transfer (Gaspari and Sądowski [262]), such as

$$
\left(P_{\text {out }} \equiv \varepsilon_{\mathrm{m}} \dot{M}_{\mathrm{BH}} c^{2}\right)=\left(P_{\text {OUT }} \equiv \varepsilon_{\mathrm{M}} \dot{M}_{\mathrm{cool}} c^{2}\right) \sim L_{\text {cool }},
$$

with $L_{\text {cool }}$ the cooling luminosity (see Section 3.1). The discrepancy between the above macro and the larger micro efficiency is crucial: it implies that most of the accreted matter $\left(\dot{M}_{\text {out }} / \dot{M}_{\text {cool }}=\left(1-\varepsilon_{\mathrm{M}} / \varepsilon_{\mathrm{m}}\right)>90 \%\right)$ is re-ejected back by the SMBH, as discussed above, driven mostly in the kinetic form of UFOs and relativistic jets. Such AGN outflows/jets propagate and percolate deeper into the meso-scale atmosphere and start to entrain progressively more IGrM, loading part of the surrounding gas mass and decreasing their velocity down to several $1000 \mathrm{~km} \mathrm{~s}^{-1}$ (e.g., Giovannini [272], Fiore et al. [273]).

The last missing tile of the self-regulated cycle is the macro AGN feedback depositiona strongly debated topic since the launch of Chandra and XMM-Newton telescopes, as their angular resolution is mostly limited to the macro scale (Figure 9, top-right inset). While numerous physical mechanisms have been proposed to compensate macro cooling flows (e.g., McNamara and Nulsen [109]), heren we focus on the physics of the three major mechanisms that have been firmly established to be present in the majority of hot halos, particularly the IGrM (see the observational evidences in Section 3), namely: buoyant bubbles, shocks, and turbulence. While previous reviews tried to assess what is the dominant or sole driver of the AGN feedback, we show that the macro AGN feedback deposition is a strong nonlinear composition of at least three key processes. We can dissect such non-linearity and sub-processes via the local enstrophy analysis, which we define as the squared magnitude of the flow vorticity $\epsilon=\frac{1}{2}|\omega|^{2} \equiv \frac{1}{2}|\nabla \times \mathbf{v}|^{2}$. Neglecting the 
small dissipation term, the Lagrangian (tracer particle) framework leads to the following enstrophy evolution decomposition (Wittor and Gaspari [239]):

$$
\frac{d \epsilon}{d t}=\underbrace{-2 \epsilon(\boldsymbol{\nabla} \cdot \mathbf{v})}_{F_{\mathrm{com}}}+\underbrace{2 \epsilon\left(\frac{\omega}{|\boldsymbol{\omega}|} \cdot \nabla\right) \mathbf{v} \cdot \frac{\omega}{|\omega|}}_{F_{\mathrm{str}}}+\underbrace{\frac{\omega}{\rho^{2}} \cdot(\nabla \rho \times \nabla P)}_{F_{\mathrm{bar}}},
$$

where the three right-hand-side (positive/negative) terms are compressions/rarefactions, stretching/squeezing motions, and baroclinicity, respectively.

The more that we want to zoom into the detailed processes of the AGN heating/weather, the more we need to rely on nonlinear HD simulations. Figure 13 shows a typical AGN-heating dominated period taken from a self-regulated AGN feedback simulation of meso AGN outflows/jets that consistently balance the macro cooling flow down to $1-10 \% \dot{M}_{\text {cool,pure }}$ (Gaspari et al. [156]). The 30 million tracer particles injected on top of the Eulerian grid can dissect the major components of the macro AGN feedback. First, the progressively slower and entrained outflow/jet inflates a pair of underdense cavities/bubbles, as its ram pressure is balanced by the surrounding IGrM thermal pressure (e.g., Brighenti et al. [274]). Such bubbles are often—albeit not universally—traced by radio synchrotron emission spilling from the micro/meso jets (see Section 3.2). Within their ellipsoidal volumes $V_{\mathrm{b}}$ (Shin et al. [107]), they contain a substantial amount of enthalpy $E_{\mathrm{cav}} \simeq 4 P_{\mathrm{b}} V_{\mathrm{b}}$ in the purely relativistic case (see Section 3.1); dividing by the buoyancy time (Churazov et al. [111]), the related cavity power/heating rate is $H_{\text {cav }}=E_{\text {cav }} / t_{\text {buoy }}$. Second, the bubbles are often encased within a cocoon shock, which is the result of the strong compressional motions of the expanding outflow and bubbles (see the thick blue contours in the second panel of Figure 13). At this stage, the shock Mach number has become already weakly transonic, $\mathcal{M} \sim 1-2$ (see Figure 4); as the AGN outflow recurrently ignites, they generate a series of weak shock ripples in the IGrM (Randall et al. [14], Liu et al. [115]), which heat the gas non-adiabatically via cumulative entropy jumps, with heating rate $H_{\text {shock }}=\left(e_{\text {th }} \Delta \ln K\right) / t_{\text {age }}$ (where $e_{\text {th }}$ is the specific thermal energy and $t_{\text {age }}^{-1}$ is the frequency of shocks). Figure 13 shows that both processes are indeed present, although the relative heating ratio varies as a function of time, with shock heating being initially more vigorous toward the inner regions, while cavity deposition is more effective at $10-100 \mathrm{kpc}$ radii.

Without the third component-subsonic turbulence (see also Section 4.1)—the final macro AGN feedback deposition would be either highly anisotropic (bubble pairs) or localized (thin shock jumps). Figure 13 (third panel) shows that the AGN feedback induces major turbulence/vorticity in a quasi isotropic manner. While the jet direction is a continuous source of enhanced turbulence, the whole IGrM core experiences a quasi irreducible level of turbulent motions $\left(\sigma_{v} \sim 100-300 \mathrm{~km} \mathrm{~s}^{-1} ; \mathcal{M}_{t} \lesssim 0.5\right)$. At the same time, the simulation shows that the (negative) rarefactions avoid the runaway accumulation of large vorticity by balancing the (positive) stretching term in a volume-filling way. The final panel finally shows that baroclinicity is negligible during the macro AGN feedback deposition, as subsonic turbulence is able to preserve the alignment of density and pressure gradients. It is important to note that, while turbulence provides a key source of isotropic mixing (with characteristic scale $t_{\text {eddy }}$; Section 4.1), its subsonic nature implies that the heating rate $\left(H_{\text {turb }}=\frac{1}{2} M_{\text {gas }} \sigma_{v}^{2} / t_{\text {turb }} \propto \sigma_{v}^{3}\right.$, where $t_{\text {turb }}=t_{\text {eddy }} / \mathcal{M}_{t}^{2} ;$ Gaspari et al. [275]) is not only a fraction of the global cooling rate, but it also has a substantially delayed deposition time $t_{\text {turb }} \gg t_{\text {cool }}[237,276]$. Alternatively, reorienting jets similar to the case of NGC 5044 (see Figure 8) may provide an alternative way of heating the gas in a quasi-isotropic way. Cielo et al. [277] presented simulations of AGN/IGrM interaction in the case of precessing jets and claimed that the distributed energy is sufficient for offsetting cooling and reproducing the features seen in real cool-core clusters. 


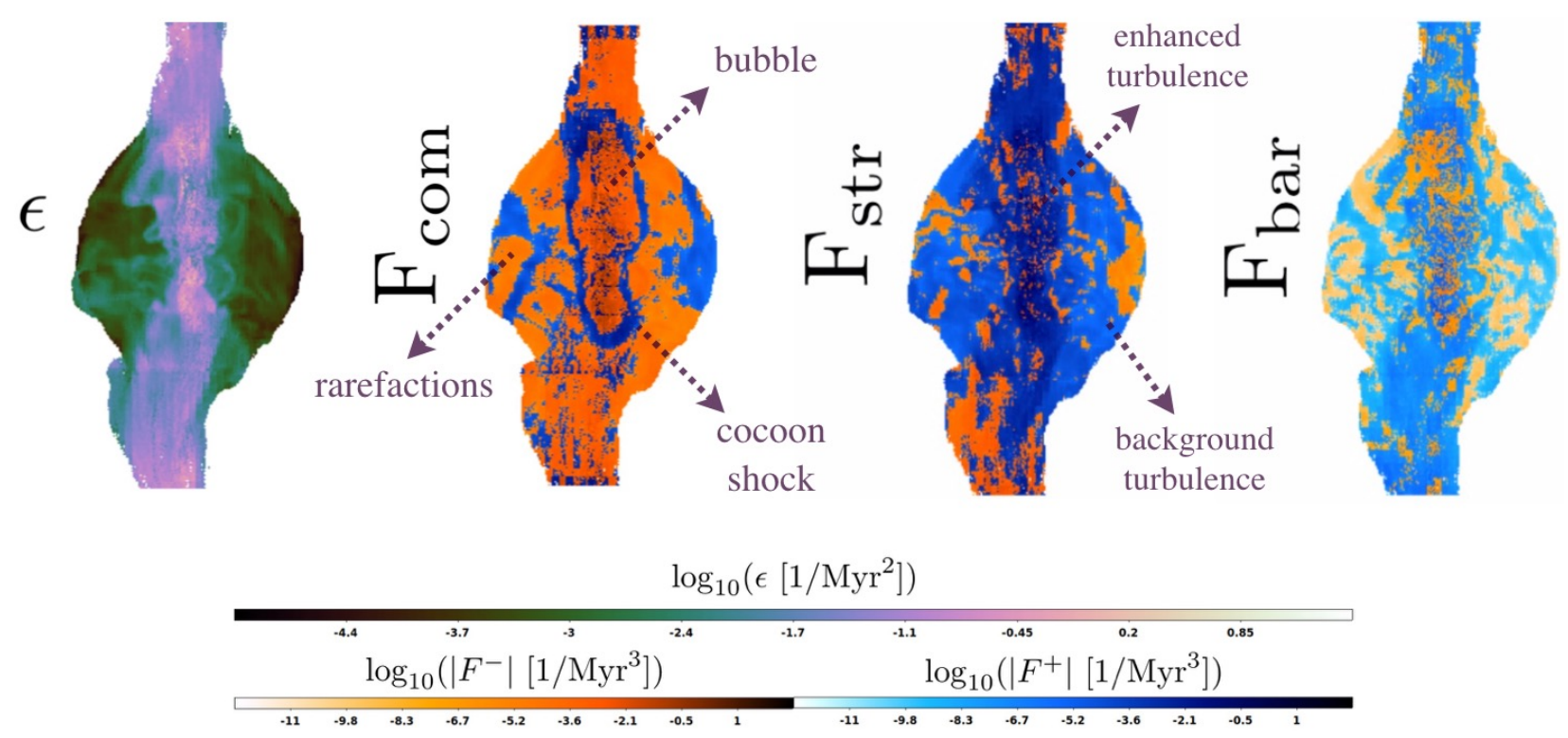

Figure 13. The macro AGN feedback transfer and deposition highlighted via the enstrophy $\epsilon$ decomposition (Equation (17)) into its main positive/negative components: compressions/rarefactions (second panel), stretching/squeezing motions (third), baroclinicity (fourth)_adapted from Wittor and Gaspari [239]. This is achieved via Lagrangian tracer particles on top of an adaptive-mesh-refinement HD simulation of self-regulated AGN outflows/jets in a central massive galaxy (Gaspari et al. [156]). The cycle of CCA rain, AGN outflow injection, bubble inflation, cocoon shock expansion, and turbulence cascade repeats self-similarly over several billion years, recurrently quenching the macro cooling flow.

In closing this theoretical section, we remark a few remaining important differences between galaxy groups and the more massive clusters. While we have discussed above that the IGrM shall be strongly self-regulated to avoid overheating/overcooling, this does not imply that groups are less variable than clusters. Indeed, the tails of the chaotic feeding/feedback loop can generate relatively more disruptive imprints in the less bound IGrM (e.g., Voit et al. [278]). This is reflected in the increased morphological diversity of groups (Sun et al. [137]) and larger intrinsic scatter of the scaling relations toward the lowmass regime, as found in the fundamental $L_{\mathrm{X}}-T_{\mathrm{X}}$ (Goulding et al. [279]; see the companion Lovisari et al. review) and $M_{\mathrm{BH}}-T_{\mathrm{X}}$ (see Section 5.3) relations. While, in absolute values, the AGN deposition radius is significantly larger in galaxy clusters (up to several $100 \mathrm{kpc}$ ), normalized to function of $R_{500}$ the AGN feedback outliers can pierce through relatively larger regions of the less bound IGrM (e.g., Grossová et al. [203]). Interestingly, many elliptical galaxies (including non-centrals) show the presence of a mini-cool core with a size of $\sim 1 \mathrm{kpc}$, which could represent the irreducible inner CCA condensation region, enabling the more frequent self-regulated AGN feedback discussed above for galaxy groups.

\section{Impact of AGN Feedback on Large Scales}

\subsection{AGN Feedback in Cosmological Simulations}

Hydrodynamical cosmological simulations are paramount for self-consistently modelling the highly non-linear formation of large-scale structure. They can simultaneously precisely solve for the gravitational and hydrodynamical aspects of structure formation. Yet, because these simulations have limited spatial and mass resolutions, one needs to implement simplified 'sub-grid' prescriptions for including crucial physical processes, such as cooling, star formation, and the feedback from supernovae and AGN, since these phenomena take place at scales that cannot be resolved by the simulations. For a complete review on numerical simulations of galaxy groups, we refer the reader to Oppenheimer et al. within this issue. Modern simulations of galaxy groups broadly fall into three categories: 
1. High resolution zoom simulations (a few hundred pc spatial resolution and $\sim 10^{5} M_{\odot}$ particles) of a few groups or of a small volume. These types of simulations are typically used to develop new baryonic physics and study the details of its impact on the IGrM (e.g., ROMULUS [280]; NewHorizon [281]; FABLE [282]).

2. Moderate resolution simulations (spatial resolution of the order of $\sim 1 \mathrm{kpc}$ and $\sim 10^{6} M_{\odot}$ particles) of volumes that are large enough (about $100 \mathrm{Mpc}$ on a side) to contain a sizable sample of groups, but not many clusters (e.g., Horizon-AGN [283]; EAGLE [284]; Illustris (TNG) [285,286]; SIMBA [287,288]; MassiveBlack-II [289]).

3. Low resolution simulations (about $5 \mathrm{kpc}$ spatial resolution and $\sim 10^{9} \mathrm{M}_{\odot}$ particles) of much larger volumes ( 300-1000 Mpc on a side with the most common value being around $500 \mathrm{Mpc}$ ) to contain a large sample of groups and clusters (e.g., IllustrisTNG300 [286]; cosmo-OWLS [12]; BAHAMAS [41]; Magneticum [290]; Horizon Run $5[291])$.

These simulations have been run with codes that use different methods for solving the equations of hydrodynamics in a cosmological context. Namely, the Tree Particle Mesh (TreePM) and smoothed particle hydrodynamics (SPH) code GADGET [292] in various versions for the majority (EAGLE, MassiveBlack-II, cosmo-OWLS, BAHAMAS, Magneticum), the moving mesh codes AREPO [293,294], and GIZMO [295] for a smaller number (FABLE, Illustris(TNG), SIMBA), and, finally, the adaptive mesh refinement (AMR) code RAMSES [296] for an even smaller fraction of them (Horizon-AGN, Horizon Run 5, and NewHorizon). Note that ROMULUS was run with the Tree+SPH code CHANGA [297]. All of these simulations include a sophisticated modelization of the non-gravitational processes of galaxy formation, such as metal-dependent radiative cooling, star formation, chemical evolution, accretion onto supermassive black holes, and feedback processes from supernovae, asymptotic giant branch stars, as well as AGN. Some of them have even calibrated the free parameters of these models on observations (e.g., FABLE, EAGLE, Illustris(TNG), and BAHAMAS). Note that the value of these parameters are often at least informed by higher-resolution simulations.

In the majority of cases, cosmological simulations (type 2 and 3) implement some variation of the Booth and Schaye [298] AGN feedback model (hereafter BS09), which is itself largely based upon the Springel et al. [292] model (hereafter S05). In the BS09 model, halos are seeded with BH seeds in their center when their mass, as evaluated by an on-the-fly halo finder, first reaches $M_{\mathrm{h}, \min }=100 m_{D M}$, where $m_{D M}$ is the mass of a dark matter particle (as in [21]). At that point, $\mathrm{BH}$ seeds are introduced at the bottom of the potential well, with masses $M_{\text {seed }}=0.001 m_{g}$, where $m_{g}$ is the mass of a gas particle. $\mathrm{BH}$ can then grow either by gas accretion or mergers. Specifically, $\mathrm{BH}$ accrete from the surrounding gas at a rate that is proportional to that given by the Eddington-limited Bondi-Hoyle-Lyttleton [299,300] formula,

$$
\dot{M}_{a c c}=\alpha \dot{M}_{\text {Bondi }}=\alpha \frac{4 \pi G^{2} M_{\mathrm{BH}}^{2} \rho}{\left(c_{s}^{2}+v^{2}\right)^{3 / 2}},
$$

where $v$ is the velocity of the $\mathrm{BH}$ relative to the ambient gas. The dimensionless 'boosting' factor $\alpha$ was introduced by S05 as a numerical correction factor that attempts to correct for the limitations of numerical simulations (see also Section 4.1). It was independent of density and had a constant value of 100 (e.g., [22,292,301-303]). BS09 introduced a density-dependent efficiency that varies as a power law of the density with a power-law index $\beta=2$ when the density is above $n_{H}^{*}=0.1 \mathrm{~cm}^{-3}$ and $\beta=1$ otherwise. BondiHoyle accretion is spatially resolved when the local gas density $n_{H}^{\star}<0.1 \mathrm{~cm}^{-3}$, which corresponds to the threshold for the formation of a cold $\left(T \lesssim 10^{4} \mathrm{~K}\right)$ phase, and when the simulations resolve the Jeans length (see BS09 and e.g., [304] for a detailed discussion). The $\mathrm{BH}$ growth rate can then be determined from the mass accretion rate by assuming a given radiative efficiency $\epsilon_{r}, \dot{M}_{\mathrm{BH}}=\dot{M}_{a c c}\left(1-\epsilon_{r}\right)$. The total radiative efficiency is always assumed to be 10 per cent, which is the mean value for the radiatively efficient [305] 
accretion on to a Schwarzschild BH. Bondi accretion-albeit very simple to implement in subgrid models - is far from a realistic representation of the feeding processes (such as CCA and multiphase precipitation), thus we advocate for fundamental updates of subgrid models in future works, as discussed in Section 4.1.

$\mathrm{BH}$ inject a fixed fraction of the rest-mass energy of the gas that they accrete into the surrounding medium. The feedback is only implemented thermally. In that case, the energy is deposited into the surrounding gas by increasing its internal energy, as opposed to kinetic feedback, which deposits energy by kicking the gas. The fraction of the accreted rest-mass energy that is injected is assumed to be independent of both the environment and accretion rate (i.e., no distinction between 'quasar mode' and 'radio mode' feedback as in the models of e.g., [302], which still injected energy thermally in both cases). The amount of energy returned by a $\mathrm{BH}$ to its surrounding medium in a time-step $\Delta t$ is given by

$$
E_{\mathrm{feed}}=\epsilon_{f} \epsilon_{r} \dot{M}_{\mathrm{BH}} c^{2} \Delta t
$$

where $\epsilon_{f}$ is the efficiency with which a BH couples the radiated energy into its surroundings (a free parameter) and $c$ is the speed of light. In order to ensure that the thermal feedback from BHs is efficient, and that it is not immediately radiated away, BS09 introduced a minimum heating temperature $\Delta T_{\min }$. BHs store feedback energy until they have accumulated an energy $E_{\text {crit }}$ that is large enough to increase the temperature of a number $n_{\text {heat }}$ of their neighbours by an amount of $\Delta T_{\min }$, which is,

$$
E_{\text {crit }}=\frac{n_{\text {heat }} m_{g} k_{B} \Delta T_{\text {min }}}{(\gamma-1) m_{H}} .
$$

The internal energy of the heated gas is instantaneously increased by $E_{\text {crit }}$. If $\Delta T_{\min }$ is set too low, the cooling time of the heated gas remains very short and the energy is efficiently radiated away. If $n_{\text {heat }} \Delta T_{\min }$ is set too high, then the energy threshold and the time period between AGN heating events become very large. Thus, $n_{\text {heat }} \Delta T_{\min }$ is connected to the AGN duty cycle. The energy is then isotropically deposited into the gas.

The recent increase in resolution (from category 3 to category 2) led to improvements of the AGN feedback modeling, as more physical processes could be taken into account. For instance, the EAGLE team modified the BS09 formula for $\dot{M}_{a c c}$ differently from what had been previously done by BS09 to take the angular momentum of the gas accreted by the $\mathrm{BH}$ into account, such that the above accretion rate was multiplied by a factor $\alpha=\min \left(1, C_{\text {visc }}^{-1}\left(c_{S} / V_{\phi}\right)^{3}\right)$, where $V_{\phi}$ is the rotation speed of the gas around the $\mathrm{BH}$ [306] and $C_{\text {visc }}$ is a free parameter that $\mathrm{s}$ related to the viscosity of the accretion disc. Improvements to the [302] model used by [22] have also been made as part of the Illustris, IllustrisTNG and FABLE projects for the same reasons. Specifically, the authors added a third mode of AGN feedback (i.e., the feedback is now thermal, mechanical, and radiative, as described in [307]) for Illustris. The kinetic AGN feedback model in the low accretion rate regime was updated for IllustrisTNG [308] (see [309] for an exhaustive discussion of the changes between Illustris and IllustrisTNG). In parallel, the FABLE team also modified the Illustris model to alleviate some of its shortcomings, such as the underestimation of the gas fractions of groups and clusters (see Section 5.2, and, in particular, the discussion of Figures 14 and 15 below). The parameters of the feedback model were calibrated on the gas mass fractions using a strategy similar to the one employed for BAHAMAS ([41]; see [282] for detailed discussion). In particular, being inspired by the BS09 model, they introduced a $25 \mathrm{Myr}$ duty cycle for the AGN feedback to reduce artificial overcooling. We note that both Horizon-AGN [283] and NewHorizon [281] use two modes of feedback, as originally introduced by [302], but that the low accretion rate or 'radio' mode is kinetic instead of thermal (the detailed modelling can be found in [310]). Indeed, the observed and realistic astrophysical deposition of heating in hot halos is carried out most of the time via the AGN outflows and jets, as discussed in Section 4.2. 


\subsection{The Hot Gas Fraction and the AGN Feedback Model}

The total baryon content and its partition between the various gas and stellar phases put fundamental constraints on galaxy formation models and, in particular, on the strength of AGN feedback, as stated in Section 3.1.4. Thus, here we present the gas mass fraction$M_{500}$ relation at $z=0$ for two compilations of massive galaxies, groups and cluster simulations that include different sorts of baryonic physics: (i) historical simulations, which is, simulations run before 2014-2015 in Figure 14; and, (ii) modern simulations run as from 2014-2015 that calibrated the free parameters of their subgrid models to reproduce at least the galaxy stellar mass function at $z=0$ in Figure 15. The various simulation sets will be compared with the compilation of observations that we presented in Section 3.1.4 and especially Figure 7, which is shown as a gray band on both figures.

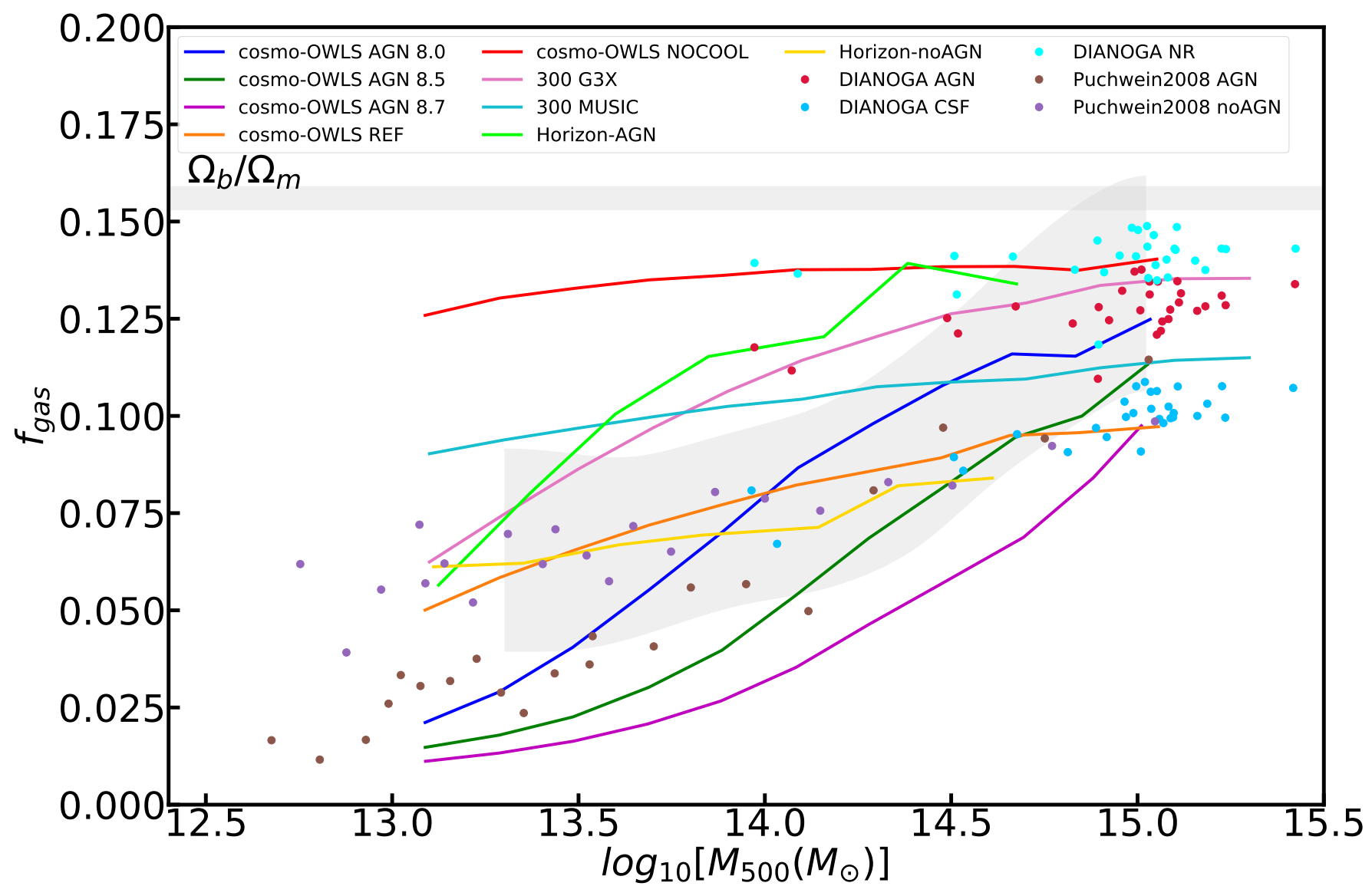

Figure 14. Compilation of historical simulation results for the gas fraction within $R_{500}$ as a function of $M_{500}$. The red, orange, magenta, green and dark blue solid lines correspond to the different sub-grid models of cosmo-OWLS [12], the pink and cyan ones to the 300 clusters run with the GADGET3X [311] and MUSIC [312] codes, respectively, as part of The Three Hundred Project [313], the lime and gold ones correspond to Horizon-AGN and, its counterpart without AGN, Horizon-noAGN [283], the cyan, blue, and crimson ones to the various physical models of the DIANOGA suite [314] and, finally, the purple and brown symbols correspond to the simulations of [22] without and with AGN, respectively. The compilation of observations presented in Figure 7 is shown as a gray band.

In Figure 14, we present the results of a compilation of historical simulations for the gas fraction within $R_{500}$ as a function of $M_{500}$. The NOCOOL model of cosmo-OWLS and the NR model of DIANOGA both correspond to classical non-radiative simulations, where one includes hydrodynamics, but do not allow the gas to cool through radiative processes. The REF model of cosmo-OWLS, the CSF model of DIANOGA, the 300 clusters run with the MUSIC code, as well as the noAGN models of [22] and the Horizon suite, all include prescriptions for radiative cooling, star formation, and stellar feedback, but not 
for AGN feedback. As first noted by [22,315], the inclusion of AGN feedback substantially lowers the gas fractions of both groups and clusters.The intensity and, thus, the duty cycle of the AGN feedback, as parameterized by $\Delta T_{\min }$ in BS09 (see Equation (20)), can be used to eject more or less gas from the potential well, as can be seen by comparing the models AGN 8.0, 8.5, and 8.7 of cosmo-OWLS. Here the number corresponds to the logarithm of the value of $\Delta T_{\min }$ chosen, i.e., 8.0 corresponds to $\Delta T_{\min }=10^{8} \mathrm{~K}$. The REF, CSF, and noAGN models also yield reasonable gas mass fractions, but the relation with mass is flatter than observed, because the SF efficiency does not strongly depend on halo mass. The low gas fractions in these models are achieved by overly efficient star formation (e.g., [12,315] and Figure 1). Note that, while the cosmo-OWLS models that include AGN feedback use the BS09 AGN feedback model summarized in Section 5.1, which is fully thermal, Horizon-AGN, DIANOGA and 300 G3X resort to a mixture of thermal and kinetic feedback, as originally developed by [310] for the former and [316] latter two.

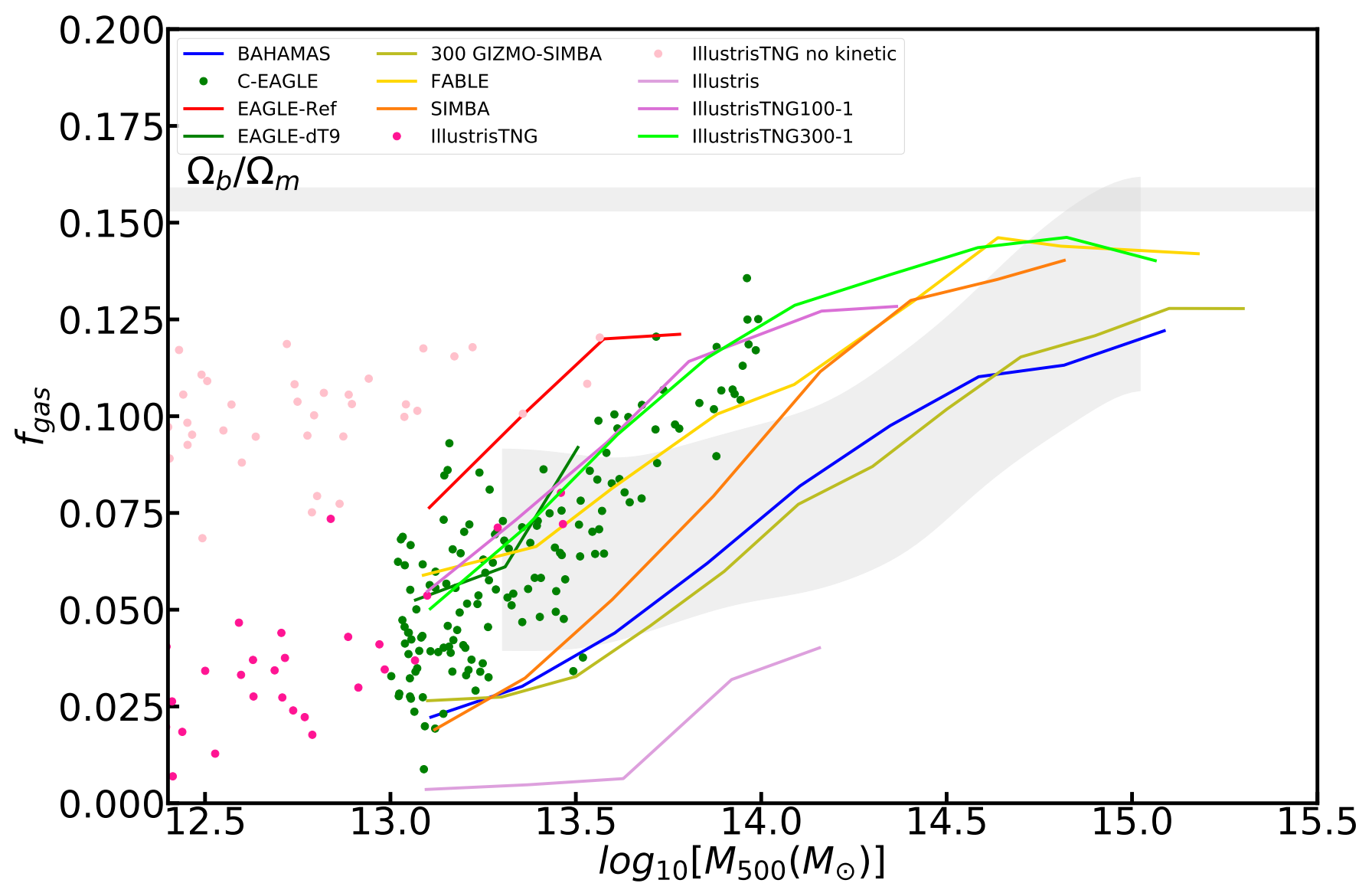

Figure 15. Compilation of gas fractions within $R_{500}$ from modern simulations as a function of $M_{500}$. The blue line corresponds to BAHAMAS [41], the red and green lines to the Reference and dT9 models of EAGLE [284], the green symbols to C-EAGLE and Hydrangea [317,318], as it uses the same sub-grid model as EAGLE-dT9, the olive line to the 300 clusters run with the GIZMO-SIMBA code (Cui et al. in preparation) as part of The Three Hundred Project [313], the salmon line to Horizon-AGN [283], the gold one to FABLE [282], the orange one to SIMBA [287,288], and the pink and deep pink symbols, as well as the plum, orchid, and lime lines correspond to various models from the Illustris and IllustrisTNG suites [285,286]. The compilation of observations presented in Figure 7 is shown as a gray band.

In Figure 15, we present the results of a compilation of modern simulations for the gas fraction within $R_{500}$ as a function of $M_{500}$. Despite the fact that most modern simulations have been calibrated to reproduce the local galaxy stellar mass function (see Figure 1), the predictions on the hot gas fraction are vastly different. For instance, Illustris (plum line) vastly underpredicts the observed hot gas fractions, whereas the reference EAGLE model 
(red line) clearly overpredicts them. Therefore, a setup that broadly reproduces the stellar content of galaxies in the Universe may simultaneously fail at reproducing the properties of the hot gas phase. Note that, in the case of BAHAMAS (blue line) and FABLE (gold line), the free parameters of the stellar and AGN feedback have been adjusted to reproduce both the $z=0$ galaxy stellar mass function and gas content of groups and clusters (see discussions in [41,282]). IllustrisTNG and EAGLE-dT9 (and the associated simulations) are versions of Illustris and EAGLE in which the AGN feedback parameters were slightly adjusted to reduce the discrepancies with the gas content of massive groups and clusters. It is worth noting that SIMBA uses a fully kinetic AGN feedback model [287], while the simulations from the Illustris series and FABLE include a mix of thermal, kinetic/mechanical, and radiative feedback $[282,285,309]$ in the vein of the one first developed by [302].

Generally speaking, we stress that the hot gas fraction of galaxy groups is an extremely sensitive probe of the feedback scheme implemented in cosmological simulations. Modern simulation suites have little predictive power on the baryon content of groups, even when the properties of the galaxy population are accurately reproduced (see Figure 1). Some of the simulations are actually calibrated on the gas mass fractions, i.e., the parameters governing the feedback model were tuned to produce reasonable gas fractions in the group regime. Major observational (Section 3.1.4) and theoretical advances (Section 4) are required to understand the ejection of baryons from halos by AGN feedback and inform the mainstream galaxy evolution models.

\subsection{Co-Evolution between the IGrM and the Central AGN}

$\mathrm{SMBH}$ masses are known to correlate with the properties of their host galaxy, in particular the integrated K-band luminosity $L_{K}$ and the velocity dispersion of the stars in the bulge, $\sigma_{\mathrm{e}}$ (see [64] for a review), as discussed in Section 2.2. However, it is still unclear whether the optical scaling relations of $\mathrm{SMBH}$ are fundamental or derive from correlations with other key quantities. Recent findings have instead unveiled that the $\mathrm{SMBH}$ masses are more tightly correlated with the properties of the host $\mathrm{X}$-ray gaseous halos, especially in the IGrM regime [76-78]. In Figure 16, we summarize our current knowledge of the relation between $\mathrm{SMBH}$ mass and $\mathrm{X}$-ray temperature within the core of galaxy groups ( $R \lesssim 0.15 R_{500}$ ). It is important to note that the SMBH masses shall be directly observed via dynamical measurements to properly unveil intrinsic scaling relations. The largest existing study is provided by Gaspari et al. [77] with 85 systems with measured SMBH masses, most of which with temperatures $\sim 0.5-1 \mathrm{keV}$ that were typical of galaxy groups. A Bayesian fit to the relation finds slopes $M_{\mathrm{BH}} \propto T_{\mathrm{x}}^{2.1}$ (Figure 16, green) and $M_{\mathrm{BH}} \propto L_{\mathrm{x}}^{0.4}$. At the high-mass end, Bogdán et al. [76] measure a somewhat flatter slope, $M_{\mathrm{BH}} \propto T_{\mathrm{x}}^{1.7}$. Notably, the intrinsic scatter goes down to $\sim 0.2$ dex, with a very high correlation coefficient at or above the 0.9 level, when compared to $\sim 0.5$ dex for the K-band luminosity. The correlations hold, regardless of the large diversity of systems, from BGGs and ETGs to non-central lenticular/spiral galaxies. Varying the extraction radius by slightly enlarging or decreasing it (group outskirts or CGM) does not significantly vary such conclusions (see the companion Lovisari et al. review for the complementary $R_{500}$ scalings, such as $M_{\mathrm{BH}}-L_{500}$ and $M_{\mathrm{BH}}-M_{\mathrm{tot}}$ ). We note that multi-variate X-ray correlations (a.k.a. 'fundamental planes') do not further improve the intrinsic scatter. In sum, by comparing the different $\mathrm{X}$-ray/optical scaling relations, it has emerged that the extended plasma (collisional) atmospheres seem to play a more fundamental role than small-scale (collisionless) stellar properties in the co-evolution of SMBH and groups. This is further supported by zoom-in cosmological simulations [319-321]. On the other hand, the slope (and scatter) of the current cosmological simulations still remain too low when compared with the observations (dotted lines in Figure 16), indicating the need to model more realistic feeding and feedback physics (see Section 4) into the coarse subgrid numerical modules. 


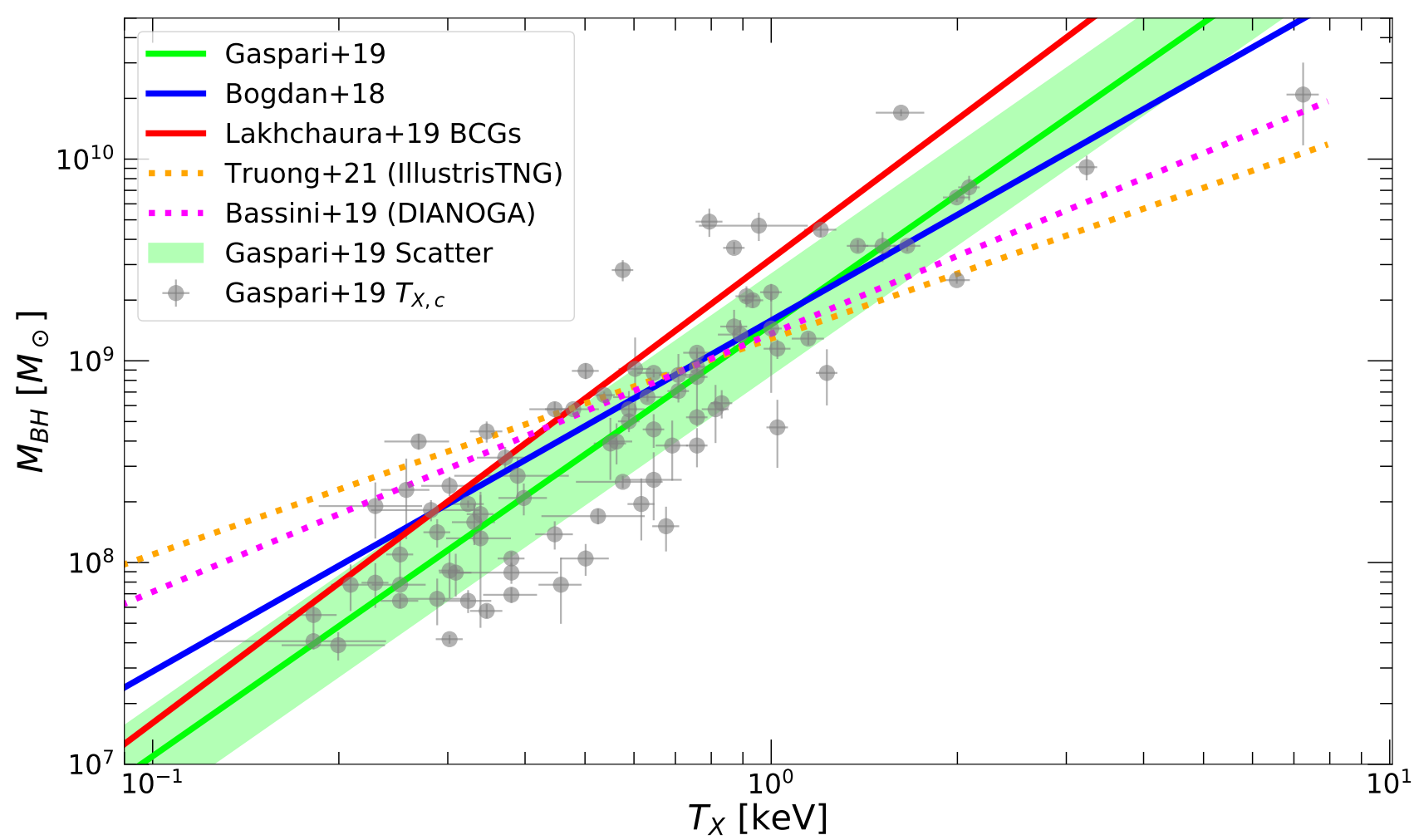

Figure 16. Relation between $\mathrm{BH}$ mass and IGrM X-ray temperature. The data points show dynamical measurements of $\mathrm{BH}$ mass plotted against the spectroscopic X-ray temperature of the host halo. The solid curves show the fitted observational relations from Gaspari et al. [77] (green; together with the quoted log-normal scatter), Bogdán et al. [76] (blue), and the "BCG" subsample of Lakhchaura et al. [78] (red). The dashed lines show the predictions of cosmological simulations with AGN feedback (Illustris TNG, Truong et al. [321]; DIANOGA, Bassini et al. [319]). The gray data points are taken from the sample of Gaspari et al. [77], which already included the smaller samples that were used by Bogdán et al. [76] and Lakhchaura et al. [78].

The above SMBH versus X-ray correlations are crucial for testing models of galaxy / group formation and evolution. Accretion/feeding models can be broadly divided into cold and hot accretion modes, as discussed in Section 4.1. Besides the cosmic dawn, hierarchical binary $\mathrm{BH}$ mergers are a present, but subdominant growth channel over most of cosmic time $[77,319]$. In hot accretion (usually Bondi or Advection Dominated Accretion FlowADAF; Bondi [249], Narayan and Fabian [250]), the larger the thermal entropy of the gas, the more strongly feeding is stifled, since the inflowing gas has to overcome the outward thermal pressure of the hot halo. This would induce negative correlations with the IGrM properties, which are ruled out by the strongly positive correlations that are shown in Figure 16. Conversely, cold-mode accretion (Gaspari et al. [245], Voit [246]; Section 4.1) typically in chaotic form (due to the turbulent condensation out of the IGrM generating randomly colliding clouds) - is linearly and tightly correlated with the X-ray luminosity and gas mass. Figure 17 (left) shows the CCA final mass growth via theoretical/numerical predictions [77] when compared to direct measurements of SMBH masses. During the Gyr evolution, the turbulent IGrM locally condenses into extended warm filaments and cold molecular clouds via nonlinear thermal instability. The clouds inelastic collisions at the meso/kpc scale boost the micro accretion rate down to the Schwarzschild radius, hence triggering strong AGN feedback heating. Such recurrent SMBH growth drives the $M_{\mathrm{BH}}$ that is shown in Figure 17, with excellent agreement with the SMBH mass observed in our local universe. 

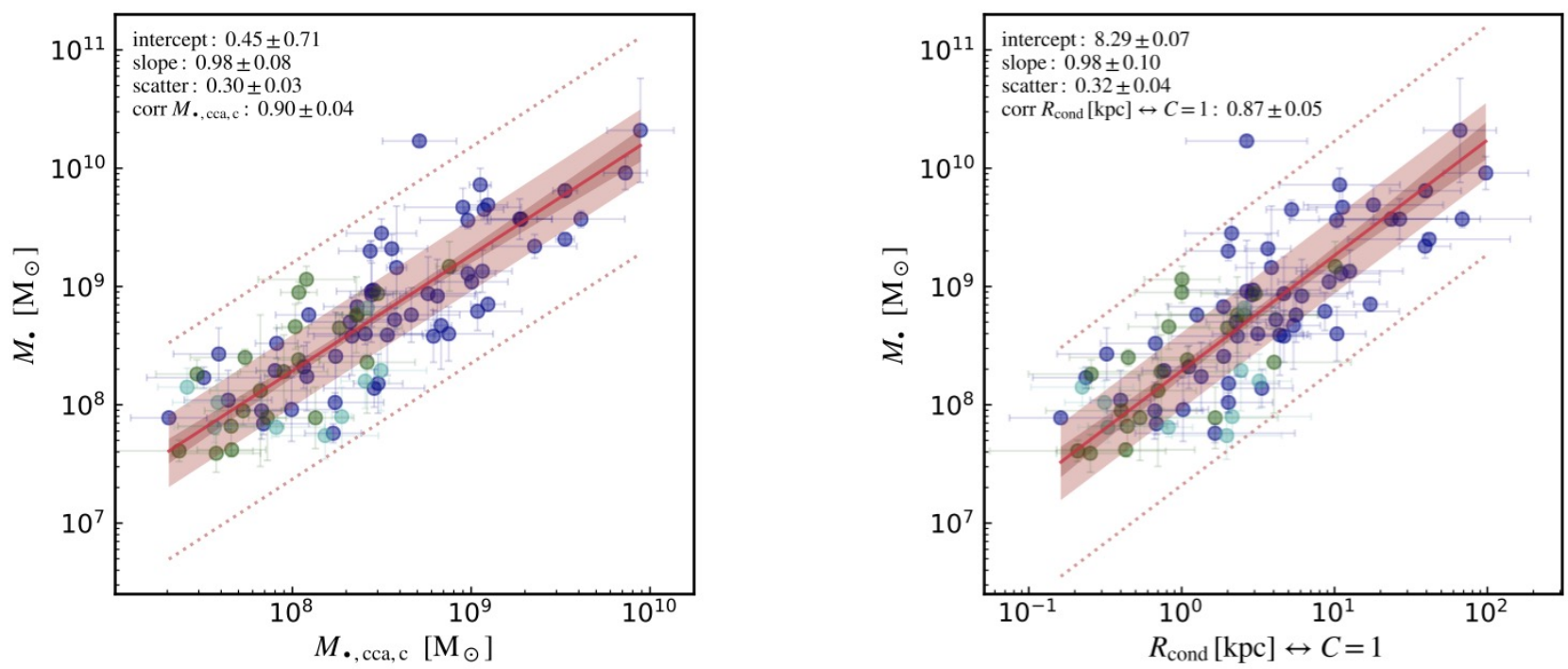

Figure 17. X-ray scaling relations are key to constrain different baryonic physics of the IGrM, here in terms of feeding models. Left: Direct SMBH masses plotted against the mass derived from theoretical/numerical predictions of chaotic cold accretion (CCA) via the X-ray core properties-adapted from Gaspari et al. [77]. Right: Hot-halo condensation radius as a function of $\mathrm{BH}$ mass and thus IGrM halo mass (the locus where $C \equiv t_{\text {cool }} / t_{\text {eddy }}=1$; see Section 3.1.3 and Section 4.1). See Figure 12 for the description of the analysis, color coding, and sample. Figure reproduced from Gaspari et al. [77].

Scaling relations allow us to predict other baryonic physics of the IGrM, such as the extent of the IGrM multiphase condensation radius, which is shown in the right panel of Figure 17. As introduced in Section 4.1, such a radius is the locus at which the IGrM cooling time and the turbulent eddy-turnover time match $\left(C \equiv t_{\text {cool }} / t_{\text {eddy }}=1\right)$, both of which can be retrieved via the $\mathrm{X}$-ray scaling relations as a function of $T_{\mathrm{x}}$ and $L_{\mathrm{x}}$ (Gaspari et al. [77]). Evidently, lower mass groups have condensation radii of less than a few kpc, while massive groups can reach $R_{\text {cond }}$ of a few $10 \mathrm{kpc}$ (e.g., David et al. [209], Olivares et al. [167], Lakhchaura et al. [206]). Overall, scaling relations between X-ray macro-scale properties translates into scaling relations of micro-scale properties $\left(M_{\mathrm{BH}}\right)$, corroborating a tight co-evolution between multi-scale processes in the IGrM (as depicted in Figure 9).

\subsection{Impact on Cosmological Probes}

During the past few years, it has become clear that AGN feedback will play an important role as a leading source of systematic uncertainties for upcoming high-profile cosmology experiments. Indeed, the energy that is injected by the central AGN affects the global distribution of baryons (see Section 5.2), leading to local depletion or excesses of matter with respect to the expectations of models, including dark matter only. This effect is most important in galaxy groups, since these systems correspond to the peak of the local halo mass density and their baryonic properties are highly sensitive to feedback. As a result, the matter power spectrum at $z=0$ can be substantially altered by baryonic physics and, in particular, AGN feedback (e.g., [322,323]). The shape of the power spectrum is strongly affected by baryonic processes on scales $k \gtrsim 10^{-1} \mathrm{~h} / \mathrm{Mpc}$. For $10^{-1}<k<10 \mathrm{~h} / \mathrm{Mpc}$, most of the simulations predict a deficit of power with respect to the N-body case, although the actual amplitude of the effect is highly uncertain [324]. The evacuation of baryons from the central regions of galaxy groups under the influence of feedback is responsible for the deficit of power on scales of $\sim 1 \mathrm{Mpc}$, i.e., roughly the typical size of galaxy groups. On smaller scales $(k \gtrsim 10 \mathrm{~h} / \mathrm{Mpc})$, cooling and condensation of baryons in the central regions lead to a rapidly increasing power. 
Accurately predicting the shape of the matter power spectrum is crucial for the success of future cosmic shear experiments, such as Euclid, which aim at determining the growth of structures by measuring the matter power spectrum and its evolution [325]. Semboloni et al. [323] showed that neglecting baryonic effects would imply important systematics on the determination of cosmological parameters. Systematic effects can be mitigated by excluding the small scales $\left(k \gtrsim 10^{-1} h / \mathrm{Mpc}\right)$ when fitting the measured power spectra, although that comes at the price of greatly increased uncertainties in the resulting cosmological parameters. Constraints on extended cosmologies, such as massive neutrinos, variable dark energy equation of state, or chameleon gravity, require sensitivity on smaller scales, and their effect is strongly degenerate with that of baryonic physics [326,327].

Chisari et al. [324] showed that numerical simulations have not yet converged on the actual impact of feedback on the power spectrum (see their Figure 3). For instance, the very strong feedback that was implemented in the original Illustris simulation, which was sufficient to completely evacuate the gas content from most groups (see Figure 15), leads to a very strong suppression of power $(>30 \%)$ on scales of a few hundred kpc. Conversely, simulations implementing a more gentle feedback scheme (EAGLE, HorizonAGN, MassiveBlackII) predict small corrections with respect to the fiducial DM-only case for $k \lesssim 10 \mathrm{~h} / \mathrm{Mpc}$. These simulations also predict a high gas fraction in galaxy groups (see Section 5.2 and [328] for an extensive discussion). Recently, Schneider et al. [329] used a semi-analytic model to predict the impact of baryons on the matter power spectrum based on the observed gas properties of groups. The authors modified the mass profiles of halos in large N-body simulations to account for star formation and AGN feedback. In particular, the semi-analytic model of Schneider et al. [329] is highly sensitive to the parameter $\theta_{e j}$, which governs the ejection of gas from the central regions of the halo by AGN feedback. In Figure 18, we show how the predicted matter power spectrum depends on $\theta_{e j}$. With increasing feedback, a progressively larger fraction of the gas is ejected from the halo and, thus, the expected power gets more strongly suppressed. Calibrating their semi-analytic model on the observed gas fraction and gas density profiles of group-scale halos, Schneider et al. [329] provided a range of predictions matching the existing observational constraints. High-precision measurements of the gas density profiles in a representative sample of galaxy groups would allow us to precisely determine the expected shape of the power spectrum [327], thereby providing a key input for upcoming cosmology experiments.

In addition to the matter power spectrum, AGN feedback on the scales of galaxy groups also affects several other cosmological observables, such as the thermal SZ power spectrum (e.g., [330,331]). Indeed, AGN feedback affects the pressure profiles of halos and thus modifies the amplitude of the power spectrum on small scales ( $\ell \gtrsim 1000$, [332]). Ramos-Ceja et al. [333] showed that tSZ models based on the universal pressure profile [334] overpredict the power measured by SPT and ACT on small scales. A strong feedback scenario and a low gas fraction on group scales are needed to fit the measured power. The effect of feedback also implies modifications to the cross-correlations between the tSZ and other observables (e.g., [335,336]). Finally, the choice of the feedback scheme affects the shape of the halo mass function (e.g., [337-339]) and the structure of dark-matter halos (e.g., $[337,340])$. We refer to the companion Oppenheimer et al. review for a more general discussion of the topic. 


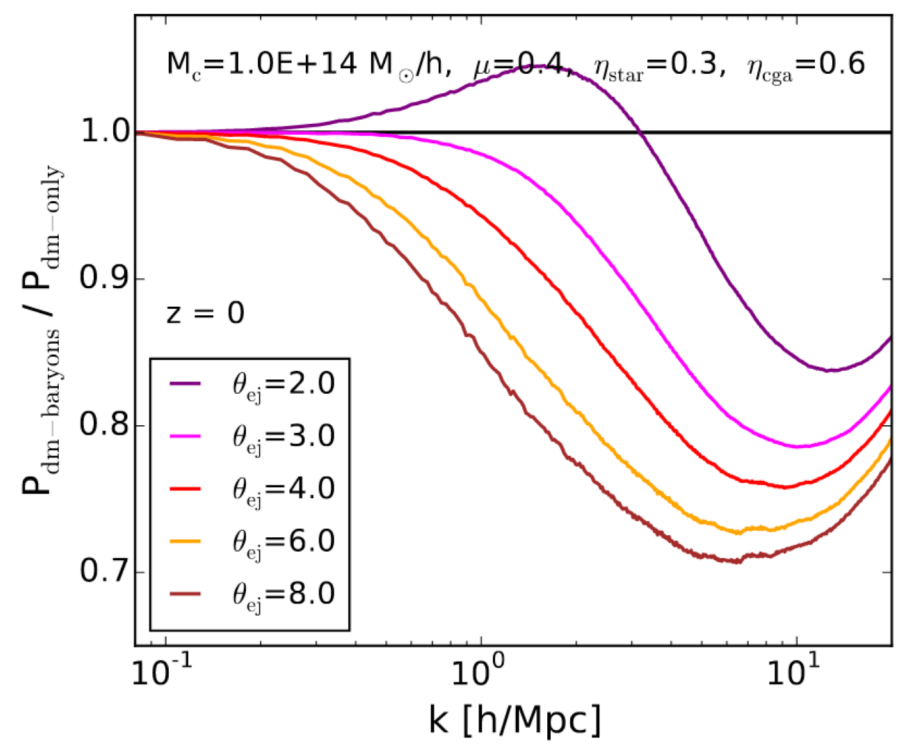

Figure 18. Modification of the local matter power spectrum with respect to pure N-body simulations in the presence of AGN feedback at the scale of galaxy groups in the semi-analytic model of Schneider et al. [329]. The various curves show how the power spectrum depends on the parameter $\theta_{e j}$ governing gas ejection from the central regions of groups under the influence of AGN feedback. A strong ejection of gas from the core of halos implies substantial modifications to the matter power spectrum on scales of $\sim 1 \mathrm{Mpc}$. The figure reproduced from Schneider et al. [329].

\section{Future Observatories}

\section{1. eROSITA}

At present, our knowledge of the population of galaxy groups in the local Universe comes largely from the ROSAT All-Sky Survey (RASS). Groups that were identified in the RASS (or even the Einstein slew survey) form the basis of most studies of the mechanics of AGN feedback at this mass scale, but, unfortunately, groups are at the lower limit of sensitivity for these surveys. RASS is therefore biased toward the detection of relaxed, centrally-concentrated, cool-core systems, with the strength of the bias increasing as mass decreases from poor clusters to groups [185]. Searches tailored to the detection of more extended sources in RASS reveal a population of low surface brightness groups undetected by the original survey [341] confirming the expected bias, while XMM-Newton observations of optically-selected groups identify both low luminosity and disturbed systems previously not detected or not recognised as groups in RASS [106].

Spectrum Roentgen Gamma (SRG, launched in 2019) hosts the eROSITA instrument, a set of seven co-aligned soft X-ray telescopes covering the $0.2-10 \mathrm{keV}$ band, with a field of view of $1^{\circ}$ and $\sim 15^{\prime \prime}$ spatial resolution. SRG will spend four years surveying the whole sky once every six months, with eROSITA building up a map $\sim 20 \times$ deeper than RASS in the $0.5-2 \mathrm{keV}$ band [342]. This is sufficient to detect essentially every galaxy group with a virialized halo in the local universe [106]. More massive groups with luminosities $\sim 10^{42} \mathrm{erg} \mathrm{s}^{-1}$ should be detectable to $z \sim 0.1$ in the final eRASS:8 survey. Käfer et al. [343] performed detailed simulations to evaluate the sensitivity of eRASS:8 to galaxy groups. The authors used a wavelet decomposition algorithm sensitive to large-scale diffuse emission. In Figure 19, we show the corresponding sensitivity curves for two possible source detection setups: a decomposition over wavelets of scales $1-4^{\prime}$ that were optimized for relatively compact sources, and the other for scales in the range 1-16' sensitive to the most extended nearby sources. Using these setups, the authors predict eRASS:8 will detect all the galaxy groups with $M_{500}>10^{13} M_{\odot}$ out to $z=0.05$. The most massive groups $\left(\sim 10^{14} M_{\odot}\right)$ will be detected out to $z=0.5$. While the survey observations will typically only provide luminosity and morphology information for individual halos, the 
group samples derived from them will be a solid base from which to investigate the impact of cooling and AGN feedback in groups, particularly when combined with radio surveys.

Pointed observations with eROSITA, possible once the survey phase is complete, may also prove useful for studies of groups. The combination of a large field of view and soft band effective area (roughly double that of the XMM-Newton EPIC-pn) is well-suited to observations of the outskirts of nearby groups, and the search for cavities or other structures that are associated with giant group-central radio galaxies. Thanks to its short focal length and very stable background [344], eROSITA is very sensitive to diffuse X-ray emission below $2 \mathrm{keV}$, which means that it is well suited to study the diffuse $\mathrm{X}$-ray emission of galaxy groups.

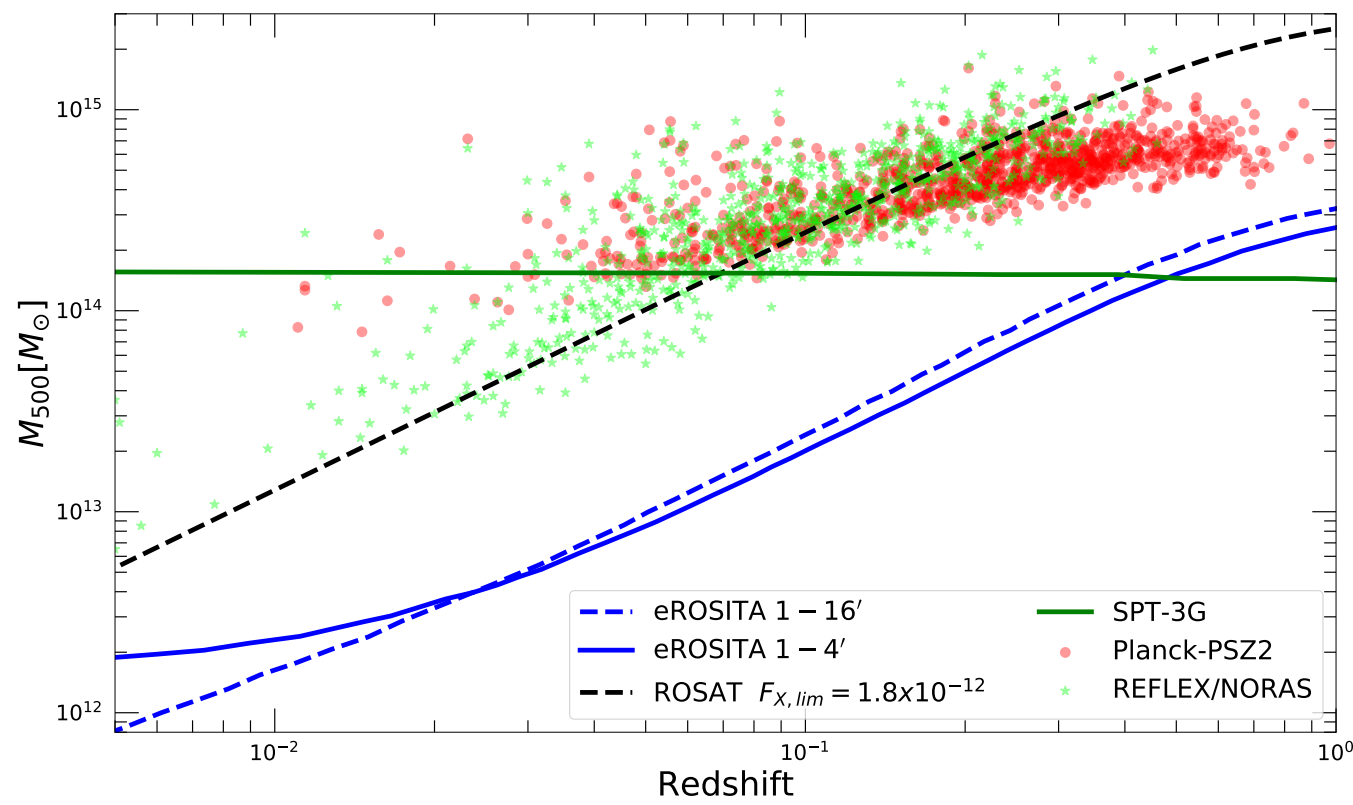

Figure 19. Expected sensitivity curve of the final eROSITA survey (eRASS:8) compared to the sensitivity of existing and upcoming X-ray and SZ surveys. The eROSITA sensitivity curve was computed from synthetic data using a wavelet decomposition algorithm [343] sensitive to scales of $1-4^{\prime}$ (solid blue curve) and 1-16' (dashed blue curve). For comparison, the dashed black curve shows the sensitivity of the ROSAT all-sky survey assuming a fixed soft X-ray flux threshold of $1.8 \times 10^{-12} \mathrm{erg} \mathrm{s}^{-1}$, which is the typical sensitivity of the REFLEX and NORAS samples (green asterisks, [345]). The red points show the systems selected from the second Planck SZ catalogue [346]. The green curve shows the expected sensitivity of the SPT-3G experiment [347].

\subsection{XRISM}

The X-ray Imaging and Spectroscopy Mission (XRISM), expected to launch by April 2023, will open a new era of high spectral-resolution observations of galaxy groups. Its X-ray microcalorimeter (Resolve, [348]) will have a constant $7 \mathrm{eV}$ energy resolution across its 0.3-12 keV band, resolving the forest of emission lines that characterizes emissions from the IGrM. This offers opportunities in a number of important areas, including measurements of bulk flows and turbulence in the hot gas. At present, grating spectra only provide upper limits on the turbulence of the IGrM [240,349], with possible hints of asymmetries that are associated with sloshing motions [350]. Hitomi demonstrated the capabilities of microcalorimeters, but it was only able to make a single turbulence measurement in the Perseus cluster [351]. XRISM should be able to measure turbulent velocities down to tens of $\mathrm{km} \mathrm{s}^{-1}$, providing a measure of the kinetic energy stored in the IGrM and allowing us to determine how much of the energy of AGN outbursts can be diffused out into the IGrM by these gas motions. The spectra from the Resolve microcalorimeter will also provide a detailed view of shock heating and cooling, with individual emission lines accurately 
tracing gas at different temperatures. Performance verification targets for the mission include NGC 5044 and NGC 4636, and, while the spatial resolution ( $>1^{\prime}$ ) and effective area of the observatory may limit its use to relatively bright nearby groups, its results are likely to be ground-breaking.

\subsection{Athena}

The Advanced Telescope for High Energy Astrophysics (Athena), which is expected to launch in the early 2030s, represents the next generation of major X-ray observatories, with $5^{\prime \prime}$ spatial resolution and an effective area of $1.4 \mathrm{~m}^{2}$ at $1 \mathrm{keV}$ (roughly $45 \times$ that of XRISM's Resolve instrument). It will carry a $40^{\prime} \times 40^{\prime}$ active pixel detector (the wide field imager, WFI) providing CCD-like spectral imaging, and a $5^{\prime}$-diameter microcalorimeter array (the X-ray Integral Field Unit, X-IFU) with $\leq 5^{\prime \prime}$ pixels, capable of $2.5 \mathrm{eV}$ spectral resolution (see, e.g., [352]). The combination of the very large collecting area with these two instruments will open up several new fields of study for galaxy groups. The WFI survey, performed over the first four years of operations, is expected to find $>10,000$ groups and clusters at $z \geq 0.5$, including $\sim 20$ groups with $\mathrm{M}_{500} \geq 5 \times 10^{13} \mathrm{M}_{\odot}$ at $z \sim 2$, and measure their temperature to better than $25 \%$ accuracy [353]. This will provide a clear view of the evolution of AGN feedback in groups back to the era of peak star formation and black hole growth. The identification of cavities and spectral mapping will be possible for moderating redshift, showing us the impact of feedback over the past few gigayears.

X-IFU offers capabilities that are similar to that of XRISM's Resolve, but with greatly improved spatial resolution and the ability to examine even low luminosity systems in the local universe. It will allow the mapping of turbulence and bulk flows in the IGrM, tracing gas motions that are associated with mergers, sloshing, uplift behind rising radio bubbles, or AGN-driven outflows. By mapping the kinetic and thermal energy content of the IGrM on spatial scales similar to those at which energy is injected by radio galaxies, it will allow us to quantify how much energy is injected into the hot gas by outbursts, determine where and when energy is transferred out of the radio jets and lobes, and see how it is then transported out into the surrounding halo [354]. It will also provide a clear view of the location of the coolest gas and allow us to trace the process by which it cools out of the hot phase.

\section{4. $\operatorname{Lyn} x$}

The Lynx mission concept (https:/ / www.lynxobservatory.com/report accessed on 25 April 2021, [355]) will, if approved, go beyond Athena, with sub-arcsecond resolution over a $22^{\prime} \times 22^{\prime}$ field of view and an effective area of $2 \mathrm{~m}^{2}$ at $1 \mathrm{keV}$, giving $50 \times$ the throughput of Chandra. As with Athena, an active pixel array (the high-definition X-ray imager, HDXI) would provide wide-field CCD-like spectral imaging, but with $0.3^{\prime \prime}$ pixels to take advantage of the exquisite spatial resolution. The Lynx X-ray Microcalorimeter (LXM) would bring $3 \mathrm{eV}$ spectral resolution on $1^{\prime \prime}$ spatial scales over a $5^{\prime}$ field of view, with sub-arrays offering $0.5^{\prime \prime}$ spatial resolution or $0.3 \mathrm{eV}$ spectral resolution in $1^{\prime}$ fields. Much of Lynx's proposed science relates to the detailed physics of accretion and galaxy evolution, and the X-ray universe at high redshift; the survey observations with the HDXI would be capable of detecting groups with masses as low as $2 \times 10^{13} \mathrm{M}_{\odot}$ out to a $z \sim 3$. In low-redshift systems, LXM could examine the conditions within individual cooling filaments in group cores, and measure the velocities of the weak shocks and sound waves that are produced during AGN outbursts. As with Athena, the observatory would be sensitive enough to trace the IGrM out to the virial radius in a large sample of groups, but with fine spatial resolution, making the identification of structure easier and the rejection of background sources cleaner. It is notable that Lynx would be the first mission after Chandra to be able to provide the finely detailed images that have proved to be so useful in the study of AGN feedback, reaching scales that are comparable to those of optical and radio observations. 


\subsection{The Square Kilometer Array and Its Precursors}

Radio astronomy has undergone something of a renaissance in recent years, with new and improved capabilities coming online, e.g., the upgraded Jansky Very Large Array (JVLA) and Giant Metrewave Radio Telescope (uGMRT), the Low-Frequency Array (LOFAR), and the Atacama Large Milimeter Array (ALMA). These are providing improved radio continuum surveys in the northern hemisphere and equatorial sky. However, new telescopes in southern Africa and Australia are opening up new opportunities, as they begin to survey the relatively poorly-explored southern sky. These include the Murchison Widefield Array (MWA), the Australian Square Kilometer Array Pathfinder (ASKAP), and MeerKAT in South Africa's Karoo region. The Galactic and Extragalactic All-sky MWA Survey (GLEAM, [356]) provides an early example, covering the entire southern sky below Declination $+30^{\circ}$ at frequencies $72-231 \mathrm{MHz}$. While its spatial resolution is modest $\left(\sim 100^{\prime \prime}\right)$, its high sensitivity at low frequency and provision of fluxes in multiple bands makes it a powerful tool for studying radio galaxies, particularly old, fading sources. Higher frequency $(\sim 1 \mathrm{GHz})$, higher spatial resolution $\left(8-30^{\prime \prime}\right)$ surveys of continuum emission and $\mathrm{HI}$ are becoming available from ASKAP (e.g., RACS, WALLABY, [357,358]) and MeerKAT (e.g., MIGHTEE, [359]), and these observatories are beginning to produce interesting findings on, e.g., group dynamics and evolution $[360,361]$ and the population of giant radio galaxies [197,201].

These telescopes are the precursors of the Square Kilometer Array (SKA), a set of nextgeneration telescopes that are expected to begin operations in the late 2020s, combining wide frequency coverage with unprecedented sensitivity. The SKA will be built in phases, with phase 1 consisting of two components: the SKA1-Low, covering the 50-350 MHz band, with baselines up to $65 \mathrm{~km}$ providing spatial resolution of $\sim 4^{\prime \prime}$ at $300 \mathrm{MHz}$ and sensitivity a factor of 5-10 better than LOFAR or GMRT; and the SKA1-Mid, covering $350 \mathrm{MHz}$ to $15 \mathrm{GHz}$ with resolution $0.4^{\prime \prime}$ at $1.4 \mathrm{GHz}$ and a sensitivity up to an order of magnitude better than JVLA [362]. The proposed phase 2 SKA would improve sensitivity by another order of magnitude. Much of the SKA's proposed science relates to the early universe, but it will be an extraordinary tool for studies of feedback in groups and clusters, tracing the entire AGN population to high redshift, not merely the radio-loud systems that dominate current samples [363]. SKA surveys are likely to be sensitive to sources down to $10^{22} \mathrm{~W} \mathrm{~Hz}^{-1}$ out to $z=3-4$ [364]. This offers an opportunity to detect the radio counterparts of most galaxies that are identified in current optical surveys, including essentially all group and cluster-dominant galaxies, with sufficient resolution to allow AGN and star formation emission to be disentangled, and with the wide frequency coverage that is necessary to determine the state and age of jets and lobes. Given the very large numbers of groups and clusters that are likely to be detected in the southern sky by, e.g., eROSITA, such surveys will play an important role in identifying systems with active cooling and feedback. HI observations reaching low column densities may provide another window on cooling from the IGrM. SKA will also open up the study of diffuse radio structures and magnetic fields in groups (e.g., [365]), providing constraints on rates of energy transport and conduction in the IGrM, as well as information on gas motions [366], and perhaps even on turbulence and shocks.

\subsection{Upcoming SZ Facilities}

Upcoming surveys of the cosmic microwave background (CMB), such as CCATprime [367], Simons Observatory [368], and CMB-S4 [369], will likely also play a role in advancing our understanding of AGN feedback at group scale by providing complementary information to X-ray surveys. While the thermal SZ effect (hereafter tSZ) is a steep function of mass $\left(Y_{S Z} \propto M_{500}^{5 / 3}\right)$, stacking of the $\mathrm{tSZ}$ effect over large samples (either X-ray or optically selected) can lead to a detection down to $M_{500} \sim 10^{13} M_{\odot}$. As a pilot study, Planck Collaboration et al. [370] presented the stacked tSZ signal from a large sample of SDSS galaxies selected to be central to their halo, and found that the tSZ-to-mass scaling relation extends with no break all the way down to $M_{500} \sim 10^{12.5} M_{\odot}$. However, the interpretation of 
the result is rendered difficult by the large Planck beam $\left(\sim 8^{\prime}\right)$, which dilutes the signal [371]. While detecting the tSZ signal from individual galaxy groups will be challenging for the new generation of $C M B$ survey instruments, the angular resolution of the foreseen facilities $\left(\lesssim 1^{\prime}\right)$ will be sufficient to study the distribution of the stacked tSZ signal and determine the origin of the signal identified by Planck Collaboration et al. [370]. On the other hand, large single-dish facilities such as AtLAST [372] will be sensitive enough to detect the tSZ effect from galaxy groups [373].

Recently, the kinetic SZ effect (kSZ), i.e., the Doppler shift of the CMB spectrum induced by moving electron clouds, has emerged as a promising tool for studying the baryon content of galaxy groups [374,375]. The kSZ signal is independent of the gas temperature, which makes it, in principle, more suitable than the tSZ for the study of low-mass systems. The kSZ signal cannot be detected directly by stacking CMB observations, given that the average velocity of structures with respect to the CMB rest frame vanishes. However, the kSZ signal can be measured by cross-correlating CMB maps with spectroscopic galaxy surveys, thereby fixing each system's velocity; this technique is known as the pairwise kSZ. Several recent studies reported low-significance detections of the kSZ with this technique [376-378]. These early results may indicate that the flat gas density profiles that are inferred from X-ray data (see Section 3.1.4) extend far beyond the halo's virial radius, which, if confirmed, provides a detection of the gas expelled from the central regions of halos by AGN feedback. Cross-correlating the kSZ data from future CMB experiments with large spectroscopic surveys, like DESI, may yield a detection of the pairwise kSZ at high significance [379] and possibly out to high redshifts [380].

Author Contributions: D.E.: lead author, Sections 1, 2, 3.1.3, 3.1.4, 5.2, 5.3, 5.4, 6.1 and 6.6; M.G.: Sections 3.1.3, 4 and 5.3; F.G.: Sections 3.1.1 and 3.1.2; A.M.C.L.B.: Sections 2, 5.1 and 5.2; E.O.: Sections 3.2,3.3 and 6. All authors have read and agreed to the published version of the manuscript.

Funding: M.G. acknowledges partial support by NASA Chandra GO8-19104X/GO9-20114X and HST GO-15890.020-A grants. A.M.C.L.B. is supported by a fellowship of PSL University at the Paris Observatory. E.O. acknowledges support from NASA through XMM-Newton award number 80NSSC19K1056 and Chandra award number GO8-19112A.

Institutional Review Board Statement: Not applicable.

Informed Consent Statement: Not applicable.

Data Availability Statement: The review is based on public data and/or published papers.

Acknowledgments: We deeply thank Yannick Bahé, Weigang Cui, Marco De Petris, Federico Sembolini, Yohan Dubois, Scott Kay, Alisson Pellissier, Ewald Puchwein, Elena Rasia, Dylan Robson, Marcel van Daalen, Mark Vogelsberger and Rainer Weinberger for providing data from their simulations for inclusion in Figures 14 and 15, even if some could not be included in the end. We also thank Paul Nulsen for providing cavity parameters for inclusion in Table A1, and the anonymous referees for useful comments. We thank Ming Sun, Mark Voit, Iurii Babyk, Trevor Ponman and Aurel Schneider for granting us permission to reprint some of their figures. E.O. thanks G. Schellenberger and K. Kolokythas for useful conversations. D.E. thanks A. Finoguenov for useful discussions. D.E. and A.M.C.L.B. thank Benjamin Oppenheimer for useful discussions. We are also grateful to Tony Mroczkowski, Joop Schaye and Ming Sun for sending comments on the accepted version while we were checking the proofs, which were taken into account for the published version.

Conflicts of Interest: The authors declare no conflict of interest. 


\section{Appendix A. List of the Properties of Detected AGN Cavities in Galaxy Groups}

Table A1. Properties of the cavities detected in groups. Only quantities available from the literature are included, thus for some systems the listing will be incomplete. ${ }^{1}$ Projected semi-major axis of the cavity. ${ }^{2}$ Projected semi-minor axis of the cavity. ${ }^{3}$ Projected distance from the cavity center to the core. ${ }^{4}$ Ages are reported as $t_{s}-t_{\text {buoy }}-t_{\text {refill }}$. Where only a single value is reported, this is $t_{s} .{ }^{5}$ Bolometric luminosity between 0.001 and $100 \mathrm{keV}$ inside $r_{\text {cool }}$, where the cooling time is less than 7.7 Gyr. * Additional data provided by Nulsen, P., priv. comm.

\begin{tabular}{|c|c|c|c|c|c|c|c|}
\hline Source & $\begin{array}{c}a^{1} \\
(\mathrm{kpc})\end{array}$ & $\begin{array}{c}b^{2} \\
(\mathrm{kpc})\end{array}$ & $\begin{array}{l}R^{3} \\
(\mathrm{kpc})\end{array}$ & $\begin{array}{c}p V \\
\left(10^{56} \text { ergs }\right)\end{array}$ & $\begin{array}{l}\text { Age }^{4} \\
\text { (Myr) }\end{array}$ & $\begin{array}{c}L_{\text {cool }}^{5} \\
\left(10^{42} \mathrm{ergs} / \mathrm{s}\right)\end{array}$ & Ref. \\
\hline HCG $62 \mathrm{~N}$ & 5.0 & 4.3 & 8.4 & $2.9_{-1.5}^{+4.1}$ & $18-15-31$ & $1.8 \pm 0.2$ & {$[92,126,381]$} \\
\hline HCG $62 \mathrm{~S}$ & 4.0 & 4.0 & 8.6 & $2.1_{-1.3}^{+3.7}$ & $19-16-29$ & & \\
\hline $3 \mathrm{C} 88 \mathrm{E}$ & 23 & 23 & 28 & 95 & 60 & & [115] \\
\hline 3C449 S & 13 & 13 & 39 & 14.6 & 70 & & [382] \\
\hline IC1262 N & 2.2 & 1.5 & 6.5 & 58.0 & $17-24-52$ & $3.3_{-0.3}^{+0.2}$ & [383] \\
\hline IC1262 S & 4 & 2 & 6.1 & 50.1 & $12-21-42$ & & \\
\hline NGC 5813 in SW & 0.95 & 0.95 & 1.3 & 0.11 & 1.2 & & [384] \\
\hline NGC 5813 in NE & 1.03 & 0.93 & 1.4 & 0.15 & 1.4 & & \\
\hline NGC 5813 mid SW & 3.9 & 3.9 & 7.7 & 1.53 & 7.2 & & \\
\hline NGC 5813 mid-1 NE & 2.9 & 2.2 & 4.9 & 0.93 & 4.6 & & \\
\hline NGC 5813 mid-2 NE & 2.8 & 2.4 & 9.3 & 0.41 & 8.8 & & \\
\hline NGC 5813 out SW & 5.2 & 3.0 & 22.2 & 0.6 & 20.8 & & \\
\hline NGC 5813 out NE & 8.0 & 4.4 & 18.0 & 2.6 & 17.0 & & \\
\hline IC 4296 NW & 80 & 80 & 230 & 920 & 220 & & [203] \\
\hline NGC $741 \mathrm{~W}$ & 8 & 8 & 16 & $12.2 \pm 1.2$ & 30 & & [385] \\
\hline NGC 193 & 63.4 & 47.2 & 0.0 & $22.7_{-17.7}^{+17.3}$ & $44.2-20.4-76.9$ & $0.11 \pm 0.01$ & [199] \\
\hline NGC $507 \mathrm{E}$ & 21.7 & 8.7 & 22.1 & $308_{-63}^{+4934}$ & $48.4-229-38.9$ & $1.37 \pm 0.02$ & [199] \\
\hline NGC 507 W & 13.4 & 5.0 & 11.7 & $90_{-30}^{+254}$ & $22.2-17.4-38.9$ & & \\
\hline NGC $1550 \mathrm{E}$ & 5.96 & 2.31 & 9.0 & $6.70_{-1.98}^{+10.7}$ & $12.8-27.9-37.0$ & $2.79_{-0.01}^{+0.03}$ & [193] \\
\hline NGC $1550 \mathrm{~W}$ & 4.09 & 1.88 & 14.65 & $2.00_{-0.68}^{+2.45}$ & $19.8-52.6-33.0$ & & \\
\hline NGC 4261 E & 20.94 & 15.51 & 24.82 & $31.02_{-6.15}^{+0.98}$ & $40.5-36.6-105.3$ & $0.11_{-0.01}^{+0.08}$ & {$[106,191]$} \\
\hline NGC $4261 \mathrm{~W}$ & 18.62 & 16.91 & 21.72 & $\begin{array}{l}32.78_{-6.52}^{+6.30} \\
\end{array}$ & $35.4-31.8-99.4$ & & \\
\hline NGC 4636 NE & 2.67 & 1.11 & 3.25 & $0.28_{-0.06}^{+0.40}$ & $8.8-20.2-46.8$ & $0.18 \pm 0.01$ & [199] \\
\hline NGC 4636 SE & 2.40 & 1.52 & 2.80 & $0.47_{-0.10}^{+0.28}$ & 7.6-17.0-47.2 & & \\
\hline NGC 4636 SW & 2.78 & 1.88 & 4.62 & $0.88_{-0.31}^{+0.48}$ & $11.7-31.2-62.1$ & & \\
\hline NGC 4636 NW & 2.53 & 1.32 & 3.33 & $0.38_{-0.08}^{+0.31}$ & $9.1-21.5-49.7$ & & \\
\hline NGC 4782 & 10.7 & 10.7 & 23.0 & 11.0 & $35-54-$ & & [386] \\
\hline NGC 5044 SW & 6.54 & 2.84 & 8.60 & $2.17_{-0.49}^{+2.87}$ & $21.1-14.4-35.5$ & $4.72 \pm 0.01$ & {$[106,191]$} \\
\hline NGC 5044 NW & 3.04 & 2.34 & 4.85 & $0.62_{-0.13}^{+0.49}$ & $11.4-16.4-35.5$ & & \\
\hline NGC 5044 in SW & 0.15 & 0.15 & 0.45 & 0.0007 & 1 & & {$[195,209]$} \\
\hline NGC 5044 in NE & 0.15 & 0.15 & 0.45 & 0.0007 & 1 & & {$[195,209]$} \\
\hline NGC $5098 \mathrm{~N}$ & 3.0 & 1.6 & 2.97 & 7.0 & 18 & & [387] \\
\hline NGC $5098 \mathrm{~S}$ & 3.0 & 1.6 & 2.97 & 7.0 & 18 & & \\
\hline NGC $5846 \mathrm{~N}$ & 0.74 & 0.58 & 0.64 & $0.35_{-0.13}^{+0.15}$ & $1.7-1.2-4.6$ & $0.27 \pm 0.01$ & [199] \\
\hline NGC $5846 \mathrm{~S}$ & 0.74 & 0.58 & 0.68 & $0.35_{-0.13}^{+0.15}$ & $1.8-1.4-4.8$ & & \\
\hline NGC 5903 & 16.0 & 13.0 & 24.6 & $2.3 \pm 0.10$ & 82.5 & $0.0047 \pm 0.0005$ & [388] \\
\hline NGC $6269 \mathrm{~N}$ & 5.2 & 5.2 & 10.7 & $22.4_{-9.0}^{+7.2}$ & $14.0-14.3-26.2$ & $0.78_{-0.03}^{+0.04}$ & [199] \\
\hline NGC $6269 \mathrm{~S}$ & 5.5 & 5.5 & 12.3 & $27.2_{-10.5}^{+8.2}$ & $16.1-17.1-28.9$ & & \\
\hline NGC 6338 in NE & 4.60 & 3.22 & 3.96 & $\begin{array}{l}20.38_{-4.83}^{+9.65} \\
\end{array}$ & $7.3-5.6-19.3$ & $4.56_{-0.06}^{+0.08}$ & [210] \\
\hline NGC 6338 in SW & 4.22 & 3.22 & 6.34 & $10.20_{-2.53}^{+3.85}$ & $11.4-14.7-30.8$ & & \\
\hline NGC 6338 outer & 6.49 & 4.01 & 18.21 & $12.98_{-2.88}^{+8.38}$ & $25.8-33.5-32.7$ & & \\
\hline VII Zw 700 NE & 3.96 & 2.43 & 5.54 & $0.24_{-0.11}^{+0.17}$ & $44.0-41.7-95.5$ & $0.54 \pm 0.03$ & [210] \\
\hline
\end{tabular}


Table A1. Cont.

\begin{tabular}{|c|c|c|c|c|c|c|c|}
\hline Source & $\begin{array}{c}a^{1} \\
(\mathrm{kpc})\end{array}$ & $\begin{array}{c}b^{2} \\
(\mathrm{kpc})\end{array}$ & $\begin{array}{c}R^{3} \\
\text { (kpc) }\end{array}$ & $\begin{array}{c}p V \\
\left(10^{56} \text { ergs }\right)\end{array}$ & $\begin{array}{l}\text { Age }{ }^{4} \\
\text { (Myr) }\end{array}$ & $\begin{array}{c}L_{\mathrm{cool}}^{5} \\
\left(10^{42} \mathrm{ergs} / \mathrm{s}\right)\end{array}$ & Ref. \\
\hline VII Zw 700 SW & 4.70 & 1.90 & 3.43 & $0.43_{-0.19}^{+0.65}$ & $27.3-29.3-82.8$ & & \\
\hline NGC 6868 NW & 11.7 & 11.7 & 38.7 & 1.48 & 88-107-119 & & [389] \\
\hline NGC 6868 SE & 8.14 & 8.14 & 25.3 & 1.0 & 55 & & \\
\hline A $1991 \mathrm{~N}$ & 16.8 & 5.5 & 12.4 & 496 & $18-28-68$ & 60.4 & [390] \\
\hline A 1991 S & 13.3 & 6.2 & 11.5 & 535 & $18-29-59$ & & \\
\hline A 35811 & 7.9 & 3.8 & 3.1 & & & & [105] \\
\hline A 35812 & 3.4 & 3.7 & 3.1 & & & & [105] \\
\hline NGC 5331 & 2.2 & 1.3 & 1.2 & & & & [105] \\
\hline NGC 5332 & 3.1 & 1.6 & 1.6 & & & & [105] \\
\hline NGC 4104 & 1.9 & 1.5 & 0.0 & & & & [105] \\
\hline RXC J0352.9+1941 1 & 8.0 & 5.9 & 9.3 & & & & [107] \\
\hline RXC J0352.9+1941 2 & 7.9 & 4.3 & 10.8 & & & & \\
\hline RX J0419+0225 & 1.2 & 0.9 & 1.7 & & & & [107] \\
\hline A 25501 & 18.9 & 9.3 & 10.3 & & & & [105] \\
\hline A 25502 & 10.7 & 5.9 & 7.8 & & & & [105] \\
\hline A2717 1 & 11.2 & 6.3 & 7.9 & & & & [105] \\
\hline A2717 2 & 13.4 & 5.8 & 8.4 & & & & [105] \\
\hline AS1101 1 & 21.0 & 14.7 & 24.2 & & & & [105] \\
\hline AS1101 2 & 24.1 & 15.7 & 23.6 & & & & [105] \\
\hline ESO 351-021 & 12.2 & 8.6 & 14.8 & & & & [105] \\
\hline RX J1159+5531 1 & 7.7 & 3.9 & 7.5 & & & & [105] \\
\hline RX J1159+5531 2 & 6.7 & 4.3 & 9.7 & & & & [105] \\
\hline RX J1206-0744 & 27.6 & 21.4 & 29.1 & & & & [105] \\
\hline NGC 2300 & 1.3 & 1.0 & 1.5 & & & & [105] \\
\hline UGC 50881 & 7.3 & 5.4 & 8.4 & & & & [105] \\
\hline UGC 50882 & 6.5 & 3.6 & 5.4 & & & & [105] \\
\hline NGC 777 E & 1.9 & 2.3 & 4.6 & & & 0.99 & {$[104,113]$} \\
\hline NGC 777 W & 2.1 & 2.4 & 4.0 & & & & [113] \\
\hline NGC 4235 E & 2.4 & 4.6 & 11.8 & & & & [113] \\
\hline NGC $4235 \mathrm{~W}$ & 2.1 & 2.4 & 4.0 & & & & [113] \\
\hline NGC 15531 & 4.1 & 3.5 & 4.6 & $0.42_{-0.22}^{+0.49}$ & $13.5-10.7-33.0$ & 1.72 & [104] * \\
\hline NGC 15532 & 3.5 & 2.7 & 3.3 & $0.28_{-0.13}^{+0.23}$ & $9.8-7.4-25.2$ & 1.72 & {$[104]^{\star}$} \\
\hline NGC 16001 & 0.87 & 0.82 & 1.21 & $0.15_{-0.09}^{+0.24}$ & $2.2-1.6-4.2$ & 0.12 & {$[104]^{\star}$} \\
\hline NGC 16002 & 0.83 & 0.72 & 1.42 & $0.11_{-0.06}^{+0.09}$ & $2.5-2.1-4.3$ & 0.12 & {$[104]$ * } \\
\hline NGC 36081 & 3.2 & 2.2 & 6.0 & $0.04_{-0.01}^{+0.00}$ & $18.7-15.9-26.4$ & 0.008 & {$[104]$ * } \\
\hline NGC 36082 & 2.5 & 1.7 & 5.3 & $0.02_{-0.01}^{+0.02}$ & $16.3-14.8-21.5$ & 0.008 & [104] * \\
\hline NGC 76261 & 5.6 & 3.1 & 14.4 & $0.37_{-0.14}^{+0.21}$ & $33.1-36.5-39.9$ & 0.12 & [104] * \\
\hline NGC 76262 & 1.4 & 0.7 & 3.0 & $0.03_{-0.01}^{+0.03}$ & $6.8-7.0-8.7$ & 0.12 & {$[104]$ * } \\
\hline NGC 76263 & 1.6 & 1.1 & 3.8 & $0.06_{-0.02}^{+0.04}$ & 8.7-8.8-11.5 & 0.12 & {$[104]^{\star}$} \\
\hline NGC 76264 & 4.7 & 4.0 & 16.2 & $0.36_{-0.21}^{+0.48}$ & $37.2-42.5-43.2$ & 0.12 & {$[104]$ * } \\
\hline A 262 E & 2.6 & 2.6 & 6.2 & $1.7_{-1.1}^{+3.2}$ & $11-13-20$ & & {$[92,391]$} \\
\hline
\end{tabular}

\section{References}

1. Springel, V. The cosmological simulation code GADGET-2. Mon. Not. R. Astron. Soc. 2005, 364, 1105-1134. [CrossRef]

2. Robotham, A.S.G.; Norberg, P.; Driver, S.P.; Baldry, I.K.; Bamford, S.P.; Hopkins, A.M.; Liske, J.; Loveday, J.; Merson, A.; Peacock, J.A.; et al. Galaxy and Mass Assembly (GAMA): The GAMA galaxy group catalogue $\left(\mathrm{G}^{3} \mathrm{Cv} 1\right)$. Mon. Not. R. Astron. Soc. 2011, 416, 2640-2668. [CrossRef]

3. Davidzon, I.; Ilbert, O.; Laigle, C.; Coupon, J.; McCracken, H.J.; Delvecchio, I.; Masters, D.; Capak, P.; Hsieh, B.C.; Le Fèvre, O.; et al. The COSMOS2015 galaxy stellar mass function. Thirteen billion years of stellar mass assembly in ten snapshots. Astron. Astrophys. 2017, 605, A70, [CrossRef] 
4. Behroozi, P.S.; Wechsler, R.H.; Conroy, C. The Average Star Formation Histories of Galaxies in Dark Matter Halos from z $=0-8$. Astrophys. J. 2013, 770, 57, [CrossRef]

5. $\quad$ Leauthaud, A.; Tinker, J.; Bundy, K.; Behroozi, P.S.; Massey, R.; Rhodes, J.; George, M.R.; Kneib, J.P.; Benson, A.; Wechsler, R.H.; et al. New Constraints on the Evolution of the Stellar-to-dark Matter Connection: A Combined Analysis of Galaxy-Galaxy Lensing, Clustering, and Stellar Mass Functions from $\mathrm{z}=0.2$ to $\mathrm{z}=1$. Astrophys. J. 2012, 744, 159, [CrossRef]

6. Coupon, J.; Arnouts, S.; van Waerbeke, L.; Moutard, T.; Ilbert, O.; van Uitert, E.; Erben, T.; Garilli, B.; Guzzo, L.; Heymans, C.; et al. The galaxy-halo connection from a joint lensing, clustering and abundance analysis in the CFHTLenS/VIPERS field. Mon. Not. R. Astron. Soc. 2015, 449, 1352-1379. [CrossRef]

7. Silk, J.; Rees, M.J. Quasars and galaxy formation. Astron. Astrophys. 1998, 331, L1-L4,

8. McNamara, B.R.; Nulsen, P.E.J. Heating Hot Atmospheres with Active Galactic Nuclei. Annu. Rev. Astron. Astrophys. 2007, 45, 117-175. [CrossRef]

9. Fabian, A.C. Observational Evidence of Active Galactic Nuclei Feedback. Annu. Rev. Astron. Astrophys. 2012, 50, 455-489. [CrossRef]

10. Gitti, M.; Brighenti, F.; McNamara, B.R. Evidence for AGN Feedback in Galaxy Clusters and Groups. Adv. Astron. 2012, 2012, 1-24. [CrossRef]

11. Gaspari, M.; Tombesi, F.; Cappi, M. Linking macro-, meso- and microscales in multiphase AGN feeding and feedback. Nat. Astron. 2020, 4, 10-13. [CrossRef]

12. Le Brun, A.M.C.; McCarthy, I.G.; Schaye, J.; Ponman, T.J. Towards a realistic population of simulated galaxy groups and clusters. Mon. Not. R. Astron. Soc. 2014, 441, 1270-1290. [CrossRef]

13. Gastaldello, F.; Buote, D.A.; Temi, P.; Brighenti, F.; Mathews, W.G.; Ettori, S. X-ray Cavities, Filaments, and Cold Fronts in the Core of the Galaxy Group NGC 5044. Astrophys. J. 2009, 693, 43-55. [CrossRef]

14. Randall, S.W.; Nulsen, P.E.J.; Jones, C.; Forman, W.R.; Bulbul, E.; Clarke, T.E.; Kraft, R.; Blanton, E.L.; David, L.; Werner, N.; et al. A Very Deep Chandra Observation of the Galaxy Group NGC 5813: AGN Shocks, Feedback, and Outburst History. Astrophys. J. 2015, 805, 112, [CrossRef]

15. Larson, R.B. Effects of supernovae on the early evolution of galaxies. Mon. Not. R. Astron. Soc. 1974, 169, 229-246. [CrossRef]

16. Rees, M.J.; Ostriker, J.P. Cooling, dynamics and fragmentation of massive gas clouds: Clues to the masses and radii of galaxies and clusters. Mon. Not. R. Astron. Soc. 1977, 179, 541-559. [CrossRef]

17. White, S.D.M.; Rees, M.J. Core condensation in heavy halos: A two-stage theory for galaxy formation and clustering. Mon. Not. R. Astron. Soc. 1978, 183, 341-358. [CrossRef]

18. Granato, G.L.; De Zotti, G.; Silva, L.; Bressan, A.; Danese, L. A Physical Model for the Coevolution of QSOs and Their Spheroidal Hosts. Astrophys. J. 2004, 600, 580-594. [CrossRef]

19. Bower, R.G.; Benson, A.J.; Malbon, R.; Helly, J.C.; Frenk, C.S.; Baugh, C.M.; Cole, S.; Lacey, C.G. Breaking the hierarchy of galaxy formation. Mon. Not. R. Astron. Soc. 2006, 370, 645-655. [CrossRef]

20. Croton, D.J.; Springel, V.; White, S.D.M.; De Lucia, G.; Frenk, C.S.; Gao, L.; Jenkins, A.; Kauffmann, G.; Navarro, J.F.; Yoshida, N. The many lives of active galactic nuclei: Cooling flows, black holes and the luminosities and colours of galaxies. Mon. Not. R. Astron. Soc. 2006, 365, 11-28. [CrossRef]

21. Di Matteo, T.; Colberg, J.; Springel, V.; Hernquist, L.; Sijacki, D. Direct Cosmological Simulations of the Growth of Black Holes and Galaxies. Astrophys. J. 2008, 676, 33-53. [CrossRef]

22. Puchwein, E.; Sijacki, D.; Springel, V. Simulations of AGN Feedback in Galaxy Clusters and Groups: Impact on Gas Fractions and the $\mathrm{L}_{X}$-T Scaling Relation. Astrophys. J. 2008, 687, L53-L56, [CrossRef]

23. Cowie, L.L.; Songaila, A.; Hu, E.M.; Cohen, J.G. New Insight on Galaxy Formation and Evolution From Keck Spectroscopy of the Hawaii Deep Fields. Astron. J. 1996, 112, 839, [CrossRef]

24. Shaver, P.A.; Wall, J.V.; Kellermann, K.I.; Jackson, C.A.; Hawkins, M.R.S. Decrease in the space density of quasars at high redshift. Nature 1996, 384, 439-441. [CrossRef]

25. Madau, P.; Ferguson, H.C.; Dickinson, M.E.; Giavalisco, M.; Steidel, C.C.; Fruchter, A. High-redshift galaxies in the Hubble Deep Field: Colour selection and star formation history to $\mathrm{z}_{\sim}$ 4. Mon. Not. R. Astron. Soc. 1996, 283, 1388-1404. [CrossRef]

26. Benson, A.J.; Bower, R.G.; Frenk, C.S.; Lacey, C.G.; Baugh, C.M.; Cole, S. What Shapes the Luminosity Function of Galaxies? Astrophys. J. 2003, 599, 38-49. [CrossRef]

27. Bower, R.G.; McCarthy, I.G.; Benson, A.J. The flip side of galaxy formation: A combined model of galaxy formation and cluster heating. Mon. Not. R. Astron. Soc. 2008, 390, 1399-1410. [CrossRef]

28. Silk, J. On the fragmentation of cosmic gas clouds. I. The formation of galaxies and the first generation of stars. Astrophys. J. 1977, 211, 638-648. [CrossRef]

29. Cole, S. Modeling Galaxy Formation in Evolving Dark Matter Halos. Astrophys. J. 1991, 367, 45. [CrossRef]

30. White, S.D.M.; Frenk, C.S. Galaxy Formation through Hierarchical Clustering. Astrophys. J. 1991, 379, 52. [CrossRef]

31. Blanchard, A.; Valls-Gabaud, D.; Mamon, G.A. The origin of the galaxy luminosity function and the thermal evolution of the intergalactic medium. Astron. Astrophys. 1992, 264, 365-378.

32. Katz, N. Dissipational Galaxy Formation. II. Effects of Star Formation. Astrophys. J. 1992, 391, 502. [CrossRef]

33. Kauffmann, G.; Colberg, J.M.; Diaferio, A.; White, S.D.M. Clustering of galaxies in a hierarchical universe-I. Methods and results at z=0. Mon. Not. R. Astron. Soc. 1999, 303, 188-206. [CrossRef] 
34. van Kampen, E.; Jimenez, R.; Peacock, J.A. Overmerging and mass-to-light ratios in phenomenological galaxy formation models. Mon. Not. R. Astron. Soc. 1999, 310, 43-56. [CrossRef]

35. Cole, S.; Lacey, C.G.; Baugh, C.M.; Frenk, C.S. Hierarchical galaxy formation. Mon. Not. R. Astron. Soc. 2000, 319, 168-204. [CrossRef]

36. Cole, S.; Norberg, P.; Baugh, C.M.; Frenk, C.S.; Bland-Hawthorn, J.; Bridges, T.; Cannon, R.; Colless, M.; Collins, C.; Couch, W.; et al. The 2dF galaxy redshift survey: Near-infrared galaxy luminosity functions. Mon. Not. R. Astron. Soc. 2001, 326, 255-273. [CrossRef]

37. Balogh, M.L.; Pearce, F.R.; Bower, R.G.; Kay, S.T. Revisiting the cosmic cooling crisis. Mon. Not. R. Astron. Soc. 2001, 326, 1228-1234. [CrossRef]

38. Lin, Y.T.; Mohr, J.J.; Stanford, S.A. Near-Infrared Properties of Galaxy Clusters: Luminosity as a Binding Mass Predictor and the State of Cluster Baryons. Astrophys. J. 2003, 591, 749-763. [CrossRef]

39. Yang, X.; Mo, H.J.; van den Bosch, F.C.; Jing, Y.P. A halo-based galaxy group finder: Calibration and application to the 2dFGRS. Mon. Not. R. Astron. Soc. 2005, 356, 1293-1307. [CrossRef]

40. Eke, V.R.; Baugh, C.M.; Cole, S.; Frenk, C.S.; Navarro, J.F. Galaxy groups in the 2dF Galaxy Redshift Survey: The number density of groups. Mon. Not. R. Astron. Soc. 2006, 370, 1147-1158. [CrossRef]

41. McCarthy, I.G.; Schaye, J.; Bird, S.; Le Brun, A.M.C. The BAHAMAS project: Calibrated hydrodynamical simulations for large-scale structure cosmology. Mon. Not. R. Astron. Soc. 2017, 465, 2936-2965. [CrossRef]

42. Tinker, J.; Kravtsov, A.V.; Klypin, A.; Abazajian, K.; Warren, M.; Yepes, G.; Gottlöber, S.; Holz, D.E. Toward a Halo Mass Function for Precision Cosmology: The Limits of Universality. Astrophys. J. 2008, 688, 709-728. [CrossRef]

43. Evrard, A.E.; Henry, J.P. Expectations for X-ray cluster observations by the ROSAT satellite. Astrophys. J. 1991, 383, 95-103. [CrossRef]

44. Kaiser, N. Evolution of Clusters of Galaxies. Astrophys. J. 1991, 383, 104. [CrossRef]

45. Balogh, M.L.; McCarthy, I.G.; Bower, R.G.; Eke, V.R. Testing cold dark matter with the hierarchical build-up of stellar light. Mon. Not. R. Astron. Soc. 2008, 385, 1003-1014. [CrossRef]

46. Bryan, G.L. Explaining the Entropy Excess in Clusters and Groups of Galaxies without Additional Heating. Astrophys. J. 2000, 544, L1-L5, [CrossRef]

47. Muanwong, O.; Thomas, P.A.; Kay, S.T.; Pearce, F.R. The effect of cooling and preheating on the X-ray properties of clusters of galaxies. Mon. Not. R. Astron. Soc. 2002, 336, 527-540. [CrossRef]

48. Voit, G.M.; Bryan, G.L. Regulation of the X-ray luminosity of clusters of galaxies by cooling and supernova feedback. Nature 2001, 414, 425-427. [CrossRef] [PubMed]

49. Voit, G.M.; Bryan, G.L.; Balogh, M.L.; Bower, R.G. Modified Entropy Models for the Intracluster Medium. Astrophys. J. 2002, 576, 601-624. [CrossRef]

50. Wu, X.P.; Xue, Y.J. The Effect of Radiative Cooling on the Scale Dependence of the Global Stellar and Gas Content of Groups and Clusters of Galaxies. Astrophys. J. 2002, 572, L19-L22, [CrossRef]

51. Menci, N.; Cavaliere, A. The history of cosmic baryons: X-ray emission versus star formation rate. Mon. Not. R. Astron. Soc. 2000, 311, 50-62. [CrossRef]

52. Bower, R.G.; Benson, A.J.; Lacey, C.G.; Baugh, C.M.; Cole, S.; Frenk, C.S. The impact of galaxy formation on the X-ray evolution of clusters. Mon. Not. R. Astron. Soc. 2001, 325, 497-508. [CrossRef]

53. Kay, S.T.; Thomas, P.A.; Theuns, T. The impact of galaxy formation on X-ray groups. Mon. Not. R. Astron. Soc. 2003, 343, 608-618. [CrossRef]

54. Valdarnini, R. Iron abundances and heating of the intracluster medium in hydrodynamical simulations of galaxy clusters. Mon. Not. R. Astron. Soc. 2003, 339, 1117-1134. [CrossRef]

55. Nagai, D.; Vikhlinin, A.; Kravtsov, A.V. Testing X-ray Measurements of Galaxy Clusters with Cosmological Simulations. Astrophys. J. 2007, 655, 98-108. [CrossRef]

56. Valageas, P.; Silk, J. The entropy history of the universe. Astron. Astrophys. 1999, 350, 725-742.

57. Kravtsov, A.V.; Yepes, G. On the supernova heating of the intergalactic medium. Mon. Not. R. Astron. Soc. 2000, 318, 227-238. [CrossRef]

58. Wu, K.K.S.; Fabian, A.C.; Nulsen, P.E.J. Non-gravitational heating in the hierarchical formation of X-ray clusters. Mon. Not. R. Astron. Soc. 2000, 318, 889-912. [CrossRef]

59. Martin, C.L. Properties of Galactic Outflows: Measurements of the Feedback from Star Formation. Astrophys. J. 1999, 513, 156-160. [CrossRef]

60. Fabian, A.C.; Voigt, L.M.; Morris, R.G. On conduction, cooling flows and galaxy formation. Mon. Not. R. Astron. Soc. 2002, 335, L71-L74, [CrossRef]

61. Dolag, K.; Jubelgas, M.; Springel, V.; Borgani, S.; Rasia, E. Thermal Conduction in Simulated Galaxy Clusters. Astrophys. J. 2004, 606, L97-L100, [CrossRef]

62. Pope, E.C.D.; Pavlovski, G.; Kaiser, C.R.; Fangohr, H. The effects of thermal conduction on the intracluster medium of the Virgo cluster. Mon. Not. R. Astron. Soc. 2005, 364, 13-28. [CrossRef]

63. Kauffmann, G.; Haehnelt, M. A unified model for the evolution of galaxies and quasars. Mon. Not. R. Astron. Soc. 2000, 311, 576-588. [CrossRef] 
64. Kormendy, J.; Ho, L.C. Coevolution (Or Not) of Supermassive Black Holes and Host Galaxies. Annu. Rev. Astron. Astrophys. 2013, 51, 511-653. [CrossRef]

65. Magorrian, J.; Tremaine, S.; Richstone, D.; Bender, R.; Bower, G.; Dressler, A.; Faber, S.M.; Gebhardt, K.; Green, R.; Grillmair, C.; et al. The Demography of Massive Dark Objects in Galaxy Centers. Astron. J. 1998, 115, 2285-2305. [CrossRef]

66. Ferrarese, L.; Merritt, D. A Fundamental Relation between Supermassive Black Holes and Their Host Galaxies. Astrophys. J. 2000, 539, L9-L12, [CrossRef]

67. Gültekin, K.; Richstone, D.O.; Gebhardt, K.; Lauer, T.R.; Tremaine, S.; Aller, M.C.; Bender, R.; Dressler, A.; Faber, S.M.; Filippenko, A.V.; et al. The M- $\sigma$ and M-L Relations in Galactic Bulges, and Determinations of Their Intrinsic Scatter. Astrophys. J. 2009, 698, 198-221. [CrossRef]

68. McConnell, N.J.; Ma, C.P.; Gebhardt, K.; Wright, S.A.; Murphy, J.D.; Lauer, T.R.; Graham, J.R.; Richstone, D.O. Two ten-billionsolar-mass black holes at the centres of giant elliptical galaxies. Nature 2011, 480, 215-218. [CrossRef]

69. McConnell, N.J.; Ma, C.P. Revisiting the Scaling Relations of Black Hole Masses and Host Galaxy Properties. Astrophys. J. 2013, 764, 184, [CrossRef]

70. van den Bosch, R.C.E. Unification of the fundamental plane and Super Massive Black Hole Masses. Astrophys. J. 2016, 831, 134, [CrossRef]

71. Fabian, A.C. The obscured growth of massive black holes. Mon. Not. R. Astron. Soc. 1999, 308, L39-L43, [CrossRef]

72. Cattaneo, A.; Haehnelt, M.G.; Rees, M.J. The distribution of supermassive black holes in the nuclei of nearby galaxies. Mon. Not. R. Astron. Soc. 1999, 308, 77-81. [CrossRef]

73. Saglia, R.P.; Opitsch, M.; Erwin, P.; Thomas, J.; Beifiori, A.; Fabricius, M.; Mazzalay, X.; Nowak, N.; Rusli, S.P.; Bender, R. The SINFONI Black Hole Survey: The Black Hole Fundamental Plane Revisited and the Paths of (Co)evolution of Supermassive Black Holes and Bulges. Astrophys. J. 2016, 818, 47, [CrossRef]

74. Ferrarese, L. Beyond the Bulge: A Fundamental Relation between Supermassive Black Holes and Dark Matter Halos. Astrophys. J. 2002, 578, 90-97. [CrossRef]

75. Booth, C.M.; Schaye, J. Dark matter haloes determine the masses of supermassive black holes. Mon. Not. R. Astron. Soc. 2010, 405, L1-L5, [CrossRef]

76. Bogdán, Á.; Lovisari, L.; Volonteri, M.; Dubois, Y. Correlation between the Total Gravitating Mass of Groups and Clusters and the Supermassive Black Hole Mass of Brightest Galaxies. Astrophys. J. 2018, 852, 131. [CrossRef]

77. Gaspari, M.; Eckert, D.; Ettori, S.; Tozzi, P.; Bassini, L.; Rasia, E.; Brighenti, F.; Sun, M.; Borgani, S.; Johnson, S.D.; et al. The X-ray Halo Scaling Relations of Supermassive Black Holes. Astrophys. J. 2019, 884, 169, [CrossRef]

78. Lakhchaura, K.; Truong, N.; Werner, N. Correlations between supermassive black holes, hot atmospheres, and the total masses of early-type galaxies. Mon. Not. R. Astron. Soc. 2019, 488, L134-L142, [CrossRef]

79. Fabian, A.C. Cooling Flows in Clusters of Galaxies. Annu. Rev. Astron. Astrophys. 1994, 32, 277-318. [CrossRef]

80. Kaastra, J.S.; Ferrigno, C.; Tamura, T.; Paerels, F.B.S.; Peterson, J.R.; Mittaz, J.P.D. XMM-Newton observations of the cluster of galaxies Sérsic 159-03. Astron. Astrophys. 2001, 365, L99-L103, [CrossRef]

81. Peterson, J.R.; Paerels, F.B.S.; Kaastra, J.S.; Arnaud, M.; Reiprich, T.H.; Fabian, A.C.; Mushotzky, R.F.; Jernigan, J.G.; Sakelliou, I. X-ray imaging-spectroscopy of Abell 1835. Astron. Astrophys. 2001, 365, L104-L109, [CrossRef]

82. Molendi, S.; Pizzolato, F. Is the Gas in Cooling Flows Multiphase? Astrophys. J. 2001, 560, 194-200. [CrossRef]

83. Peterson, J.R.; Fabian, A.C. X-ray spectroscopy of cooling clusters. Phys. Rep. 2006, 427, 1-39. [CrossRef]

84. Narayan, R.; Medvedev, M.V. Thermal Conduction in Clusters of Galaxies. Astrophys. J. 2001, 562, L129-L132, [CrossRef]

85. Motl, P.M.; Burns, J.O.; Loken, C.; Norman, M.L.; Bryan, G. Formation of Cool Cores in Galaxy Clusters via Hierarchical Mergers. Astrophys. J. 2004, 606, 635-653. [CrossRef]

86. ZuHone, J.A.; Markevitch, M.; Johnson, R.E. Stirring Up the Pot: Can Cooling Flows in Galaxy Clusters be Quenched by Gas Sloshing? Astrophys. J. 2010, 717, 908-928. [CrossRef]

87. Silk, J.; Djorgovski, S.; Wyse, R.F.G.; Bruzual, A. Self-regulated Cooling Flows in Elliptical Galaxies and in Cluster Cores: Is Excusively Low Mass Star Formation Really Necessary? Astrophys. J. 1986, 307, 415. [CrossRef]

88. Burns, J.O. The radio properties of cD galaxies in Abell clusters. I-An X-ray selected sample. Astron. J. 1990, 99, 14-30. [CrossRef]

89. Best, P.N.; von der Linden, A.; Kauffmann, G.; Heckman, T.M.; Kaiser, C.R. On the prevalence of radio-loud active galactic nuclei in brightest cluster galaxies: Implications for AGN heating of cooling flows. Mon. Not. R. Astron. Soc. 2007, 379, 894-908. [CrossRef]

90. Finoguenov, A.; Jones, C. Chandra Observation of M84, a Radio Lobe Elliptical Galaxy in the Virgo Cluster. Astrophys. J. 2001, 547, L107-L110, [CrossRef]

91. Vrtilek, J.M.; Grego, L.; David, L.P.; Ponman, T.J.; Forman, W.; Jones, C.; Harris, D.E. A Sharper Picture of X-ray Bright Galaxy Groups: Chandra Imaging and Spectroscopy of HCG 62 and NGC 741; American Physical Society: Albuquerque, NM, USA, 2002; p. B17.107.

92. Bîrzan, L.; Rafferty, D.A.; McNamara, B.R.; Wise, M.W.; Nulsen, P.E.J. A Systematic Study of Radio-induced X-ray Cavities in Clusters, Groups, and Galaxies. Astrophys. J. 2004, 607, 800-809. [CrossRef]

93. Forman, W.; Nulsen, P.; Heinz, S.; Owen, F.; Eilek, J.; Vikhlinin, A.; Markevitch, M.; Kraft, R.; Churazov, E.; Jones, C. Reflections of Active Galactic Nucleus Outbursts in the Gaseous Atmosphere of M87. Astrophys. J. 2005, 635, 894-906. [CrossRef] 
94. Allen, S.W.; Dunn, R.J.H.; Fabian, A.C.; Taylor, G.B.; Reynolds, C.S. The relation between accretion rate and jet power in X-ray luminous elliptical galaxies. Mon. Not. R. Astron. Soc. 2006, 372, 21-30. [CrossRef]

95. Fabian, A.C.; Sanders, J.S.; Taylor, G.B.; Allen, S.W.; Crawford, C.S.; Johnstone, R.M.; Iwasawa, K. A very deep Chandra observation of the Perseus cluster: Shocks, ripples and conduction. Mon. Not. R. Astron. Soc. 2006, 366, 417-428. [CrossRef]

96. Dunn, R.J.H.; Fabian, A.C. Investigating AGN heating in a sample of nearby clusters. Mon. Not. R. Astron. Soc. 2006, 373, 959-971. [CrossRef]

97. Jetha, N.N.; Ponman, T.J.; Hardcastle, M.J.; Croston, J.H. Active galactic nuclei heating in the centres of galaxy groups: A statistical study. Mon. Not. R. Astron. Soc. 2007, 376, 193-204. [CrossRef]

98. Croston, J.H. Jet/Environment Interactions in Low-Power Radio Galaxies. In Extragalactic Jets: Theory and Observation from Radio to Gamma Ray; Rector, T.A., De Young, D.S., Eds.; Astronomical Society of the Pacific: Girdwood, AK, USA, 2008; Volume 386, p. 335.

99. Churazov, E.; Sunyaev, R.; Forman, W.; Böhringer, H. Cooling flows as a calorimeter of active galactic nucleus mechanical power. Mon. Not. R. Astron. Soc. 2002, 332, 729-734. [CrossRef]

100. Scheuer, P.A.G. Models of extragalactic radio sources with a continuous energy supply from a central object. Mon. Not. R. Astron. Soc. 1974, 166, 513-528. [CrossRef]

101. Nulsen, P.E.J.; McNamara, B.R.; Wise, M.W.; David, L.P. The Cluster-Scale AGN Outburst in Hydra A. Astrophys. J. 2005, 628, 629-636. [CrossRef]

102. McNamara, B.R.; Nulsen, P.E.J.; Wise, M.W.; Rafferty, D.A.; Carilli, C.; Sarazin, C.L.; Blanton, E.L. The heating of gas in a galaxy cluster by X-ray cavities and large-scale shock fronts. Nature 2005, 433, 45-47. [CrossRef]

103. Nulsen, P.E.J.; Jones, C.; Forman, W.R.; David, L.P.; McNamara, B.R.; Rafferty, D.A.; Bîrzan, L.; Wise, M.W. AGN Heating Through Cavities and Shocks. In Heating versus Cooling in Galaxies and Clusters of Galaxies; Springer-Verlag series "ESO Astrophysics Symposia."; Böhringer, H., Pratt, G.W., Finoguenov, A., Schuecker, P., Eds.; Springer: Heidelberg, Germany, 2007; p. 210. [CrossRef]

104. Cavagnolo, K.W.; McNamara, B.R.; Nulsen, P.E.J.; Carilli, C.L.; Jones, C.; Birzan, L. A relationship between AGN jet power and radio power. Astrophys. J. 2010, 720, 1066-1072. [CrossRef]

105. Dong, R.; Rasmussen, J.; Mulchaey, J.S. A Systematic Search for X-ray Cavities in the Hot Gas of Galaxy Groups. Astrophys. J. 2010, 712, 883-900. [CrossRef]

106. O'Sullivan, E.; Ponman, T.J.; Kolokythas, K.; Raychaudhury, S.; Babul, A.; Vrtilek, J.M.; David, L.P.; Giacintucci, S.; Gitti, M.; Haines, C.P. The Complete Local Volume Groups Sample-I. Sample selection and X-ray properties of the high-richness subsample. Mon. Not. R. Astron. Soc. 2017, 472, 1482-1505. [CrossRef]

107. Shin, J.; Woo, J.H.; Mulchaey, J.S. A Systematic Search for X-ray Cavities in Galaxy Clusters, Groups, and Elliptical Galaxies. Astrophys. J. 2016, 227, 31, [CrossRef]

108. Kim, D.W.; Anderson, C.; Burke, D.; D’Abrusco, R.; Fabbiano, G.; Fruscione, A.; Lauer, J.; McCollough, M.; Morgan, D.; Mossman, A.; et al. Chandra Early-type Galaxy Atlas. Astrophys. J. Suppl. Ser. 2019, 241, 36. [CrossRef]

109. McNamara, B.R.; Nulsen, P.E.J. Mechanical feedback from active galactic nuclei in galaxies, groups and clusters. New J. Phys. 2012, 14, 055023, [CrossRef]

110. Abdulla, Z.; Carlstrom, J.E.; Mantz, A.B.; Marrone, D.P.; Greer, C.H.; Lamb, J.W.; Leitch, E.M.; Muchovej, S.; O’Donnell, C.; Plagge, T.J.; et al. Constraints on the Thermal Contents of the X-ray Cavities of Cluster MS 0735.6+7421 with Sunyaev-Zel'dovich Effect Observations. Astrophys. J. 2019, 871, 195, [CrossRef]

111. Churazov, E.; Brüggen, M.; Kaiser, C.R.; Böhringer, H.; Forman, W. Evolution of Buoyant Bubbles in M87. Astrophys. J. 2001, 554, 261-273. [CrossRef]

112. Bîrzan, L.; McNamara, B.R.; Nulsen, P.E.J.; Carilli, C.L.; Wise, M.W. Radiative Efficiency and Content of Extragalactic Radio Sources: Toward a Universal Scaling Relation between Jet Power and Radio Power. Astrophys. J. 2008, 686, 859. [CrossRef]

113. Panagoulia, E.K.; Fabian, A.C.; Sanders, J.S.; Hlavacek-Larrondo, J. A volume-limited sample of X-ray galaxy groups and clusters-II. X-ray cavity dynamics. Mon. Not. R. Astron. Soc. 2014, 444, 1236-1259. [CrossRef]

114. Salvatier, J.; Wiecki, T.V.; Fonnesbeck, C. Probabilistic programming in Python using PyMC3. PeerJ Comput. Sci. 2016, 2, e55. [CrossRef]

115. Liu, W.; Sun, M.; Nulsen, P.; Clarke, T.; Sarazin, C.; Forman, W.; Gaspari, M.; Giacintucci, S.; Lal, D.V.; Edge, T. AGN feedback in galaxy group 3C 88: Cavities, shock, and jet reorientation. Mon. Not. R. Astron. Soc. Lett. 2019, 484, 3376-3392. [CrossRef]

116. Tang, X.; Churazov, E. Sound wave generation by a spherically symmetric outburst and AGN feedback in galaxy clusters. Mon. Not. R. Astron. Soc. 2017, 468, 3516-3532. [CrossRef]

117. Machacek, M.; Nulsen, P.E.J.; Jones, C.; Forman, W.R. Chandra Observations of Nuclear Outflows in the Elliptical Galaxy NGC 4552 in the Virgo Cluster. Astrophys. J. 2006, 648, 947-955. [CrossRef]

118. Forman, W.; Churazov, E.; Jones, C.; Heinz, S.; Kraft, R.; Vikhlinin, A. Partitioning the Outburst Energy of a Low Eddington Accretion Rate AGN at the Center of an Elliptical Galaxy: The Recent 12 Myr History of the Supermassive Black Hole in M87. Astrophys. J. 2017, 844, 122, [CrossRef]

119. Wise, M.W.; McNamara, B.R.; Nulsen, P.E.J.; Houck, J.C.; David, L.P. X-ray Supercavities in the Hydra A Cluster and the Outburst History of the Central Galaxy's Active Nucleus. Astrophys. J. 2007, 659, 1153-1158. [CrossRef] 
120. Croston, J.H.; Kraft, R.P.; Hardcastle, M.J.; Birkinshaw, M.; Worrall, D.M.; Nulsen, P.E.J.; Penna, R.F.; Sivakoff, G.R.; Jordán, A.; Brassington, N.J.; et al. High-energy particle acceleration at the radio-lobe shock of Centaurus A. Mon. Not. R. Astron. Soc. 2009, 395, 1999-2012. [CrossRef]

121. Snios, B.; Nulsen, P.E.J.; Wise, M.W.; de Vries, M.; Birkinshaw, M.; Worrall, D.M.; Duffy, R.T.; Kraft, R.P.; McNamara, B.R.; Carilli, C.; et al. The Cocoon Shocks of Cygnus A: Pressures and Their Implications for the Jets and Lobes. Astrophys. J. 2018, 855, 71, [CrossRef]

122. Croston, J.H.; Hardcastle, M.J.; Mingo, B.; Evans, D.A.; Dicken, D.; Morganti, R.; Tadhunter, C.N. A Large-scale Shock Surrounding a Powerful Radio Galaxy? Astrophys. J. 2011, 734, L28, [CrossRef]

123. Blanton, E.L.; Randall, S.W.; Douglass, E.M.; Sarazin, C.L.; Clarke, T.E.; McNamara, B.R. Shocks and Bubbles in a Deep Chandra Observation of the Cooling Flow Cluster Abell 2052. Astrophys. J. 2009, 697, L95-L98, [CrossRef]

124. Kraft, R.P.; Birkinshaw, M.; Nulsen, P.E.J.; Worrall, D.M.; Croston, J.H.; Forman, W.R.; Hardcastle, M.J.; Jones, C.; Murray, S.S. An Active Galactic Nucleus Driven Shock in the Intracluster Medium around the Radio Galaxy 3C 310. Astrophys. J. 2012, 749, 19. [CrossRef]

125. Baldi, A.; Forman, W.; Jones, C.; Kraft, R.; Nulsen, P.; Churazov, E.; David, L.; Giacintucci, S. The Unusual X-ray Morphology of NGC 4636 Revealed by Deep Chandra Observations: Cavities and Shocks Created by Past Active Galactic Nucleus Outbursts. Astrophys. J. 2009, 707, 1034-1043. [CrossRef]

126. Gitti, M.; O'Sullivan, E.; Giacintucci, S.; David, L.P.; Vrtilek, J.; Raychaudhury, S.; Nulsen, P.E.J. Cavities and Shocks in the Galaxy Group HCG 62 as Revealed by Chandra, XMM-Newton, and Giant Metrewave Radio Telescope Data. Astrophys. J. 2010, 714, 758-771. [CrossRef]

127. Kaiser, N. Evolution and clustering of rich clusters. Mon. Not. R. Astron. Soc. 1986, 222, 323-345. [CrossRef]

128. Allen, S.W.; Fabian, A.C. The impact of cooling flows on the T_X-L_Bol relation for the most luminous clusters. Mon. Not. R. Astron. Soc. 1998, 297, L57-L62, [CrossRef]

129. Arnaud, M.; Evrard, A.E. The L_X-T relation and intracluster gas fractions of X-ray clusters. Mon. Not. R. Astron. Soc. 1999, 305, 631-640. [CrossRef]

130. Markevitch, M. The $\mathrm{L}_{X}-\mathrm{T}$ Relation and Temperature Function for Nearby Clusters Revisited. Astrophys. J. 1998, 504, 27-34. [CrossRef]

131. Tozzi, P.; Scharf, C.; Norman, C. Detection of the Entropy of the Intergalactic Medium: Accretion Shocks in Clusters, Adiabatic Cores in Groups. Astrophys. J. 2000, 542, 106-119. [CrossRef]

132. Tozzi, P.; Norman, C. The Evolution of X-ray Clusters and the Entropy of the Intracluster Medium. Astrophys. J. 2001, 546, 63-84. [CrossRef]

133. Knight, P.A.; Ponman, T.J. The properties of the hot gas in galaxy groups and clusters from $1 D$ hydrodynamical simulations-I. Cosmological infall models. Mon. Not. R. Astron. Soc. 1997, 289, 955-972. [CrossRef]

134. Ponman, T.J.; Cannon, D.B.; Navarro, J.F. The thermal imprint of galaxy formation on X-ray clusters. Nature 1999, $397,135-137$. [CrossRef]

135. Borgani, S.; Viel, M. The evolution of a pre-heated intergalactic medium. Mon. Not. R. Astron. Soc. 2009, 392, L26-L30, [CrossRef]

136. Eke, V.R.; Navarro, J.F.; Frenk, C.S. The Evolution of X-ray Clusters in a Low-Density Universe. Astrophys. J. 1998, 503, 569-592. [CrossRef]

137. Sun, M.; Voit, G.M.; Donahue, M.; Jones, C.; Forman, W.; Vikhlinin, A. Chandra Studies of the X-ray Gas Properties of Galaxy Groups. Astrophys. J. 2009, 693, 1142-1172. [CrossRef]

138. Vikhlinin, A.; Burenin, R.A.; Ebeling, H.; Forman, W.R.; Hornstrup, A.; Jones, C.; Kravtsov, A.V.; Murray, S.S.; Nagai, D.; Quintana, H.; et al. Chandra Cluster Cosmology Project. II. Samples and X-ray Data Reduction. Astrophys. J. 2009, 692, 1033-1059. [CrossRef]

139. Lloyd-Davies, E.J.; Ponman, T.J.; Cannon, D.B. The entropy and energy of intergalactic gas in galaxy clusters. Mon. Not. R. Astron. Soc. 2000, 315, 689-702. [CrossRef]

140. Ponman, T.J.; Sanderson, A.J.R.; Finoguenov, A. The Birmingham-CfA cluster scaling project-III. Entropy and similarity in galaxy systems. Mon. Not. R. Astron. Soc. 2003, 343, 331-342. [CrossRef]

141. Brighenti, F.; Mathews, W.G. Entropy Evolution in Galaxy Groups and Clusters: A Comparison of External and Internal Heating. Astrophys. J. 2001, 553, 103-120. [CrossRef]

142. Finoguenov, A.; Jones, C.; Böhringer, H.; Ponman, T.J. ASCA Observations of Groups at Radii of Low Overdensity: Implications for the Cosmic Preheating. Astrophys. J. 2002, 578, 74-89. [CrossRef]

143. Voit, G.M.; Kay, S.T.; Bryan, G.L. The baseline intracluster entropy profile from gravitational structure formation. Mon. Not. R. Astron. Soc. 2005, 364, 909-916. [CrossRef]

144. Humphrey, P.J.; Buote, D.A.; Brighenti, F.; Flohic, H.M.L.G.; Gastaldello, F.; Mathews, W.G. Tracing the Gas to the Virial Radius $\left(\mathrm{R}_{100}\right)$ in a Fossil Group. Astrophys. J. 2012, 748, 11, [CrossRef]

145. Simionescu, A.; Werner, N.; Mantz, A.; Allen, S.W.; Urban, O. Witnessing the growth of the nearest galaxy cluster: Thermodynamics of the Virgo Cluster outskirts. Mon. Not. R. Astron. Soc. 2017, 469, 1476-1495. [CrossRef]

146. Thölken, S.; Lovisari, L.; Reiprich, T.H.; Hasenbusch, J. X-ray analysis of the galaxy group UGC 03957 beyond $\mathrm{R}_{200}$ with Suzaku. Astron. Astrophys. 2016, 592, A37, [CrossRef] 
147. Johnson, R.; Ponman, T.J.; Finoguenov, A. A statistical analysis of the Two-Dimensional XMM-Newton Group Survey: The impact of feedback on group properties. Mon. Not. R. Astron. Soc. 2009, 395, 1287-1308. [CrossRef]

148. Finoguenov, A.; Davis, D.S.; Zimer, M.; Mulchaey, J.S. The Two-dimensional XMM-Newton Group Survey: $\mathrm{z}<0.012$ Groups. Astrophys. J. 2006, 646, 143-160. [CrossRef]

149. Finoguenov, A.; Ponman, T.J.; Osmond, J.P.F.; Zimer, M. XMM-Newton study of $0.012<\mathrm{z}<0.024$ groups-I. Overview of the IGM thermodynamics. Mon. Not. R. Astron. Soc. 2007, 374, 737-760. [CrossRef]

150. Mahdavi, A.; Finoguenov, A.; Böhringer, H.; Geller, M.J.; Henry, J.P. XMM-Newton and Gemini Observations of Eight RASSCALS Galaxy Groups. Astrophys. J. 2005, 622, 187-204. [CrossRef]

151. Sanderson, A.J.R.; O'Sullivan, E.; Ponman, T.J. A statistically selected Chandra sample of 20 galaxy clusters-II. Gas properties and cool core/non-cool core bimodality. Mon. Not. R. Astron. Soc. 2009, 395, 764-776. [CrossRef]

152. Cavagnolo, K.W.; Donahue, M.; Voit, G.M.; Sun, M. Intracluster Medium Entropy Profiles for a Chandra Archival Sample of Galaxy Clusters. Astrophys. J. 2009, 182, 12-32. [CrossRef]

153. Panagoulia, E.K.; Fabian, A.C.; Sanders, J.S. A volume-limited sample of X-ray galaxy groups and clusters-I. Radial entropy and cooling time profiles. Mon. Not. R. Astron. Soc. 2014, 438, 2341-2354. [CrossRef]

154. Hogan, M.T.; McNamara, B.R.; Pulido, F.A.; Nulsen, P.E.J.; Vantyghem, A.N.; Russell, H.R.; Edge, A.C.; Babyk, I.; Main, R.A.; McDonald, M. The Onset of Thermally Unstable Cooling from the Hot Atmospheres of Giant Galaxies in Clusters: Constraints on Feedback Models. Astrophys. J. 2017, 851, 66, [CrossRef]

155. Babyk, I.V.; McNamara, B.R.; Nulsen, P.E.J.; Russell, H.R.; Vantyghem, A.N.; Hogan, M.T.; Pulido, F.A. A Universal Entropy Profile for the Hot Atmospheres of Galaxies and Clusters within R 2500. Astrophys. J. 2018, 862, 39, [CrossRef]

156. Gaspari, M.; Ruszkowski, M.; Sharma, P. Cause and Effect of Feedback: Multiphase Gas in Cluster Cores Heated by AGN Jets. Astrophys. J. 2012, 746, 94, [CrossRef]

157. McCourt, M.; Sharma, P.; Quataert, E.; Parrish, I.J. Thermal instability in gravitationally stratified plasmas: Implications for multiphase structure in clusters and galaxy haloes. Mon. Not. R. Astron. Soc. 2012, 419, 3319-3337. [CrossRef]

158. Sharma, P.; McCourt, M.; Quataert, E.; Parrish, I.J. Thermal instability and the feedback regulation of hot haloes in clusters, groups and galaxies. Mon. Not. R. Astron. Soc. 2012, 420, 3174-3194. [CrossRef]

159. Voit, G.M.; Donahue, M.; Bryan, G.L.; McDonald, M. Regulation of star formation in giant galaxies by precipitation, feedback and conduction. Nature 2015, 519, 203-206. [CrossRef]

160. Voit, G.M.; Bryan, G.L.; O'Shea, B.W.; Donahue, M. Precipitation-regulated Star Formation in Galaxies. Astrophys. J. 2015, 808, L30, [CrossRef]

161. Field, G.B. Thermal Instability. Astrophys. J. 1965, 142, 531. [CrossRef]

162. Borgani, S.; Finoguenov, A.; Kay, S.T.; Ponman, T.J.; Springel, V.; Tozzi, P.; Voit, G.M. Entropy amplification from energy feedback in simulated galaxy groups and clusters. Mon. Not. R. Astron. Soc. 2005, 361, 233-243. [CrossRef]

163. Cavagnolo, K.W.; Donahue, M.; Voit, G.M.; Sun, M. An Entropy Threshold for Strong H $\alpha$ and Radio Emission in the Cores of Galaxy Clusters. Astrophys. J. 2008, 683, L107-L110, [CrossRef]

164. Lau, E.T.; Gaspari, M.; Nagai, D.; Coppi, P. Physical Origins of Gas Motions in Galaxy Cluster Cores: Interpreting Hitomi Observations of the Perseus Cluster. Astrophys. J. 2017, 849, 54, [CrossRef]

165. Gaspari, M.; McDonald, M.; Hamer, S.L.; Brighenti, F.; Temi, P.; Gendron-Marsolais, M.; Hlavacek-Larrondo, J.; Edge, A.C.; Werner, N.; Tozzi, P.; et al. Shaken Snow Globes: Kinematic Tracers of the Multiphase Condensation Cascade in Massive Galaxies, Groups, and Clusters. Astrophys. J. 2018, 854, 167, [CrossRef]

166. Gaspari, M.; Temi, P.; Brighenti, F. Raining on black holes and massive galaxies: The top-down multiphase condensation model. Mon. Not. R. Astron. Soc. 2017, 466, 677-704. [CrossRef]

167. Olivares, V.; Salome, P.; Combes, F.; Hamer, S.; Guillard, P.; Lehnert, M.D.; Polles, F.L.; Beckmann, R.S.; Dubois, Y.; Donahue, M.; et al. Ubiquitous cold and massive filaments in cool core clusters. Astron. Astrophys. 2019, 631, A22, [CrossRef]

168. Juráňová, A.; Werner, N.; Gaspari, M.; Lakhchaura, K.; Nulsen, P.E.J.; Sun, M.; Canning, R.E.A.; Allen, S.W.; Simionescu, A.; Oonk, J.B.R.; et al. Cooling in the X-ray halo of the rotating, massive early-type galaxy NGC 7049. Mon. Not. R. Astron. Soc. 2019, 484, 2886-2895. [CrossRef]

169. Eckert, D.; Ghirardini, V.; Ettori, S.; Rasia, E.; Biffi, V.; Pointecouteau, E.; Rossetti, M.; Molendi, S.; Vazza, F.; Gastaldello, F.; et al. Non-thermal pressure support in X-COP galaxy clusters. Astron. Astrophys. 2019, 621, A40, [CrossRef]

170. Gonzalez, A.H.; Zaritsky, D.; Zabludoff, A.I. A Census of Baryons in Galaxy Clusters and Groups. Astrophys. J. 2007, 666, 147-155. [CrossRef]

171. Gastaldello, F.; Buote, D.A.; Humphrey, P.J.; Zappacosta, L.; Bullock, J.S.; Brighenti, F.; Mathews, W.G. Probing the Dark Matter and Gas Fraction in Relaxed Galaxy Groups with X-ray Observations from Chandra and XMM-Newton. Astrophys. J. 2007, 669, 158-183. [CrossRef]

172. Gonzalez, A.H.; Sivanandam, S.; Zabludoff, A.I.; Zaritsky, D. Galaxy Cluster Baryon Fractions Revisited. Astrophys. J. 2013, 778, 14, [CrossRef]

173. Pratt, G.W.; Croston, J.H.; Arnaud, M.; Böhringer, H. Galaxy cluster X-ray luminosity scaling relations from a representative local sample (REXCESS). Astron. Astrophys. 2009, 498, 361-378. [CrossRef]

174. Lovisari, L.; Reiprich, T.H.; Schellenberger, G. Scaling properties of a complete X-ray selected galaxy group sample. Astron. Astrophys. 2015, 573, A118, [CrossRef] 
175. Eckert, D.; Ettori, S.; Coupon, J.; Gastaldello, F.; Pierre, M.; Melin, J.B.; Le Brun, A.M.C.; McCarthy, I.G.; Adami, C.; Chiappetti, L.; et al. The XXL Survey. XIII. Baryon content of the bright cluster sample. Astron. Astrophys. 2016, 592, A12, [CrossRef]

176. Ettori, S. The physics inside the scaling relations for X-ray galaxy clusters: Gas clumpiness, gas mass fraction and slope of the pressure profile. Mon. Not. R. Astron. Soc. 2015, 446, 2629-2639. [CrossRef]

177. Nugent, J.M.; Dai, X.; Sun, M. Suzaku Measurements of Hot Halo Emission at Outskirts for Two Poor Galaxy Groups: NGC 3402 and NGC 5129. Astrophys. J. 2020, 899, 160, [CrossRef]

178. Sun, M. Hot gas in galaxy groups: Recent observations. New J. Phys. 2012, 14, 045004, [CrossRef]

179. Croston, J.H.; Pratt, G.W.; Böhringer, H.; Arnaud, M.; Pointecouteau, E.; Ponman, T.J.; Sanderson, A.J.R.; Temple, R.F.; Bower, R.G.; Donahue, M. Galaxy-cluster gas-density distributions of the representative XMM-Newton cluster structure survey (REXCESS). Astron. Astrophys. 2008, 487, 431-443. [CrossRef]

180. Chiu, I.; Mohr, J.J.; McDonald, M.; Bocquet, S.; Desai, S.; Klein, M.; Israel, H.; Ashby, M.L.N.; Stanford, A.; Benson, B.A.; et al. Baryon content in a sample of 91 galaxy clusters selected by the South Pole Telescope at $0.2<\mathrm{z}<1.25$. Mon. Not. R. Astron. Soc. 2018, 478, 3072-3099. [CrossRef]

181. Sanderson, A.J.R.; O'Sullivan, E.; Ponman, T.J.; Gonzalez, A.H.; Sivanandam, S.; Zabludoff, A.I.; Zaritsky, D. The baryon budget on the galaxy group/cluster boundary. Mon. Not. R. Astron. Soc. 2013, 429, 3288-3304. [CrossRef]

182. Andreon, S. The stellar mass fraction and baryon content of galaxy clusters and groups. Mon. Not. R. Astron. Soc. 2010, 407, 263-276. [CrossRef]

183. Kravtsov, A.V.; Vikhlinin, A.A.; Meshcheryakov, A.V. Stellar Mass-Halo Mass Relation and Star Formation Efficiency in High-Mass Halos. Astron. Lett. 2018, 44, 8-34. [CrossRef]

184. Rasia, E.; Ettori, S.; Moscardini, L.; Mazzotta, P.; Borgani, S.; Dolag, K.; Tormen, G.; Cheng, L.M.; Diaferio, A. Systematics in the X-ray cluster mass estimators. Mon. Not. R. Astron. Soc. 2006, 369, 2013-2024. [CrossRef]

185. Eckert, D.; Molendi, S.; Paltani, S. The cool-core bias in X-ray galaxy cluster samples. I. Method and application to HIFLUGCS. Astron. Astrophys. 2011, 526, A79. [CrossRef]

186. Andreon, S.; Wang, J.; Trinchieri, G.; Moretti, A.; Serra, A.L. Variegate galaxy cluster gas content: Mean fraction, scatter, selection effects, and covariance with X-ray luminosity. Astron. Astrophys. 2017, 606, A24, [CrossRef]

187. Lin, Y.T.; Mohr, J.J. Radio Sources in Galaxy Clusters: Radial Distribution, and 1.4 GHz and K-band Bivariate Luminosity Function. ApJS 2007, 170, 71-94. [CrossRef]

188. Smolčić, V.; Finoguenov, A.; Zamorani, G.; Schinnerer, E.; Tanaka, M.; Giodini, S.; Scoville, N. On the occupation of X-ray-selected galaxy groups by radio active galactic nuclei since $\mathrm{z}=1.3$. Mon. Not. R. Astron. Soc. Lett. 2011, 416, L31-L35. [CrossRef]

189. Dunn, R.J.H.; Allen, S.W.; Taylor, G.B.; Shurkin, K.F.; Gentile, G.; Fabian, A.C.; Reynolds, C.S. The radio properties of a complete, X-ray selected sample of nearby, massive elliptical galaxies. Mon. Not. R. Astron. Soc. Lett. 2010, 404, 180. [CrossRef]

190. Kolokythas, K.; O'Sullivan, E.; Intema, H.; Raychaudhury, S.; Babul, A.; Giacintucci, S.; Gitti, M. The complete local volume groups sample-III. Characteristics of group central radio galaxies in the Local Universe. Mon. Not. R. Astron. Soc. Lett. 2019, 489, 2488-2504. [CrossRef]

191. Kolokythas, K.; O'Sullivan, E.; Raychaudhury, S.; Giacintucci, S.; Gitti, M.; Babul, A. The Complete Local Volume Groups Sample-II. A study of the central radio galaxies in the high-richness sample. Mon. Not. R. Astron. Soc. Lett. 2018, $481,1550$. [CrossRef]

192. Giacintucci, S.; O'Sullivan, E.; Vrtilek, J.M.; David, L.P.; Raychaudhury, S.; Venturi, T.; Athreya, R.M.; Clarke, T.E.; Murgia, M.; Mazzotta, P.; et al. A combined low-radio frequency/X-ray study of AGN feedback in groups of galaxies-I. GMRT observations at $235 \mathrm{MHz}$ and $610 \mathrm{MHz}$. Astrophys. J. 2011, 732, 95. [CrossRef]

193. Kolokythas, K.; O’Sullivan, E.; Giacintucci, S.; Worrall, D.M.; Birkinshaw, M.; Raychaudhury, S.; Horellou, C.; Intema, H.; Loubser, I. Evidence of AGN feedback and sloshing in the X-ray luminous NGC 1550 galaxy group. Mon. Not. R. Astron. Soc. Lett. 2020, 496, 1471-1487. [CrossRef]

194. Br̂zan, L.; Rafferty, D.A.; Bruggen, M.; Botteon, A.; Brunettit, G.; Cuciti, V.; Edge, A.C.; Morganti, R.; Rottgering, H.J.A.; Shimwell, T. LOFAR Observations of X-ray Cavity Systems. Mon. Not. R. Astron. Soc. Lett. 2020, 496, 2613-2635. [CrossRef]

195. Schellenberger, G.; David, L.P.; Vrtilek, J.; O’Sullivan, E.; Giacintucci, S.; Forman, W.; Jones, C.; Venturi, T. A New Feedback Cycle in the Archetypal Cooling Flow Group NGC 5044. Astrophys. J. 2021, 906, 16. [CrossRef]

196. Ineson, J.; Croston, J.H.; Hardcastle, M.J.; Kraft, R.P.; Evans, D.A.; Jarvis, M. The link between accretion mode and environment in radio-loud active galaxies. Mon. Not. R. Astron. Soc. Lett. 2015, 453, 2682-2706. [CrossRef]

197. Pasini, T.; Brüggen, M.; de Gasperin, F.; Bîrzan, L.; O'Sullivan, E.; Finoguenov, A.; Jarvis, M.; Gitti, M.; Brighenti, F.; Whittam, I.H.; et al. The relation between the diffuse X-ray luminosity and the radio power of the central AGN in galaxy groups. Mon. Not. $R$. Astron. Soc. Lett. 2020, 497, 2163-2174. [CrossRef]

198. Bîrzan, L.; Rafferty, D.A.; Nulsen, P.E.J.; McNamara, B.R.; Röttgering, H.J.A.; Wise, M.W.; Mittal, R. The duty cycle of radio-mode feedback in complete samples of clusters. Mon. Not. R. Astron. Soc. Lett. 2012, 427, 3468. [CrossRef]

199. O'Sullivan, E.; Giacintucci, S.; David, L.P.; Gitti, M.; Vrtilek, J.M.; Raychaudhury, S.; Ponman, T.J. Heating the Hot Atmospheres of Galaxy Groups and Clusters with Cavities: The Relationship between Jet Power and Low-frequency Radio Emission. Astrophys. J. 2011, 735, 11. [CrossRef] 
200. Giodini, S.; Smolčić, V.; Finoguenov, A.; Boehringer, H.; Bîrzan, L.; Zamorani, G.; Oklopčić, A.; Pierini, D.; Pratt, G.W.; Schinnerer, E.; et al. Radio Galaxy Feedback in X-ray-selected Groups from COSMOS: The Effect on the Intracluster Medium. Astrophys. J. 2010, 714, 218. [CrossRef]

201. Delhaize, J.; Heywood, I.; Prescott, M.; Jarvis, M.J.; Delvecchio, I.; Whittam, I.H.; White, S.V.; Hardcastle, M.J.; Hale, C.L.; Afonso, J.; et al. MIGHTEE: Are giant radio galaxies more common than we thought? Mon. Not. R. Astron. Soc. Lett. 2021, 501, $3833-3845$. [CrossRef]

202. O'Sullivan, E.; Worrall, D.M.; Birkinshaw, M.; Trinchieri, G.; Wolter, A.; Zezas, A.; Giacintucci, S. Interaction between the intergalactic medium and central radio source in the NGC 4261 group of galaxies. Mon. Not. R. Astron. Soc. Lett. 2011, 416, 2916-2931. [CrossRef]

203. Grossová, R.; Werner, N.; Rajpurohit, K.; Mernier, F.; Lakhchaura, K.; Gabányi, K.; Canning, R.E.A.; Nulsen, P.; Massaro, F.; Sun, M.; et al. Powerful AGN jets and unbalanced cooling in the hot atmosphere of IC 4296. Mon. Not. R. Astron. Soc. Lett. 2019, 488, 1917-1925. [CrossRef]

204. Cantwell, T.M.; Bray, J.D.; Croston, J.H.; Scaife, A.M.M.; Mulcahy, D.D.; Best, P.N.; Brüggen, M.; Brunetti, G.; Callingham, J.R.; Clarke, A.O.; et al. Low-frequency observations of the giant radio galaxy NGC 6251. Mon. Not. R. Astron. Soc. Lett. 2020, 495, 143-159. [CrossRef]

205. Ineson, J.; Croston, J.H.; Hardcastle, M.J.; Mingo, B. A representative survey of the dynamics and energetics of FR II radio galaxies. Mon. Not. R. Astron. Soc. Lett. 2017, 467, 1586-1607. [CrossRef]

206. Lakhchaura, K.; Werner, N.; Sun, M.; Canning, R.E.A.; Gaspari, M.; Allen, S.W.; Connor, T.; Donahue, M.; Sarazin, C. Thermodynamic properties, multiphase gas, and AGN feedback in a large sample of giant ellipticals. Mon. Not. R. Astron. Soc. 2018, 481, 4472-4504. [CrossRef]

207. Pulido, F.A.; McNamara, B.R.; Edge, A.C.; Hogan, M.T.; Vantyghem, A.N.; Russell, H.R.; Nulsen, P.E.J.; Babyk, I.; Salomé, P. The Origin of Molecular Clouds in Central Galaxies. Astrophys. J. 2018, 853, 177. [CrossRef]

208. Werner, N.; Oonk, J.B.R.; Sun, M.; Nulsen, P.E.J.; Allen, S.W.; Canning, R.E.A.; Simionescu, A.; Hoffer, A.; Connor, T.; Donahue, M.; et al. The origin of cold gas in giant elliptical galaxies and its role in fuelling radio-mode AGN feedback. Mon. Not. R. Astron. Soc. Lett. 2014, 439, 2291-2306. [CrossRef]

209. David, L.P.; Vrtilek, J.; O'Sullivan, E.; Jones, C.; Forman, W.; Sun, M. The Presence of Thermally Unstable X-ray Filaments and the Production of Cold Gas in the NGC 5044 Group. Astrophys. J. 2017, 842, 84. [CrossRef]

210. O'Sullivan, E.; Schellenberger, G.; Burke, D.J.; Sun, M.; Vrtilek, J.M.; David, L.P.; Sarazin, C. Building a cluster: Shocks, cavities, and cooling filaments in the group-group merger NGC 6338. Mon. Not. R. Astron. Soc. Lett. 2019, 488, 2925-2946. [CrossRef]

211. Gomes, J.M.; Papaderos, P.; Kehrig, C.; Vílchez, J.M.; Lehnert, M.D.; Sánchez, S.F.; Ziegler, B.; Breda, I.; Dos Reis, S.N.; IglesiasPáramo, J.; et al. Warm ionized gas in CALIFA early-type galaxies. 2D emission-line patterns and kinematics for 32 galaxies. Astron. Astrophys. 2016, 588, A68. [CrossRef]

212. Diniz, S.I.F.; Pastoriza, M.G.; Hernandez-Jimenez, J.A.; Riffel, R.; Ricci, T.V.; Steiner, J.E.; Riffel, R.A. Integral field spectroscopy of the inner kpc of the elliptical galaxy NGC 5044. Mon. Not. R. Astron. Soc. Lett. 2017, 470, 1703-1717. [CrossRef]

213. Schellenberger, G.; David, L.P.; Vrtilek, J.; O'Sullivan, E.; Lim, J.; Forman, W.; Sun, M.; Combes, F.; Salome, P.; Jones, C.; et al. Atacama Compact Array Measurements of the Molecular Mass in the NGC 5044 Cooling-flow Group. Astrophys. J. $2020,894,72$. [CrossRef]

214. McDonald, M.; Gaspari, M.; McNamara, B.R.; Tremblay, G.R. Revisiting the Cooling Flow Problem in Galaxies, Groups, and Clusters of Galaxies. Astrophys. J. 2018, 858, 45, [CrossRef]

215. Kaneda, H.; Onaka, T.; Sakon, I.; Kitayama, T.; Okada, Y.; Suzuki, T. Properties of Polycyclic Aromatic Hydrocarbons in Local Elliptical Galaxies Revealed by the Infrared Spectrograph on Spitzer. Astrophys. J. 2008, 684, 270-281. [CrossRef]

216. Rose, T.; Edge, A.C.; Combes, F.; Gaspari, M.; Hamer, S.; Nesvadba, N.; Peck, A.B.; Sarazin, C.; Tremblay, G.R.; Baum, S.A.; et al. Constraining cold accretion on to supermassive black holes: Molecular gas in the cores of eight brightest cluster galaxies revealed by joint CO and CN absorption. Mon. Not. R. Astron. Soc. 2019, 489, 349-365. [CrossRef]

217. Babyk, I.V.; McNamara, B.R.; Tamhane, P.D.; Nulsen, P.E.J.; Russell, H.R.; Edge, A.C. Origins of Molecular Clouds in Early-type Galaxies. Astrophys. J. 2019, 887, 149. [CrossRef]

218. Davis, T.A.; Greene, J.E.; Ma, C.P.; Blakeslee, J.P.; Dawson, J.M.; Pandya, V.; Veale, M.; Zabel, N. The MASSIVE survey—XI. What drives the molecular gas properties of early-type galaxies. Mon. Not. R. Astron. Soc. Lett. 2019, 486, 1404-1423. [CrossRef]

219. O'Sullivan, E.; Combes, F.; Hamer, S.; Salomé, P.; Babul, A.; Raychaudhury, S. Cold gas in group-dominant elliptical galaxies. Astron. Astrophys. 2015, 573, A111. [CrossRef]

220. O'Sullivan, E.; Combes, F.; Salomé, P.; David, L.P.; Babul, A.; Vrtilek, J.M.; Lim, J.; Olivares, V.; Raychaudhury, S.; Schellenberger, G. Cold gas in a complete sample of group-dominant early-type galaxies. Astron. Astrophys. 2018, 618, A126. [CrossRef]

221. Temi, P.; Amblard, A.; Gitti, M.; Brighenti, F.; Gaspari, M.; Mathews, W.G.; David, L. ALMA Observations of Molecular Clouds in Three Group-centered Elliptical Galaxies: NGC 5846, NGC 4636, and NGC 5044. Astrophys. J. 2018, 858, 17. [CrossRef]

222. David, L.P.; Lim, J.; Forman, W.; Vrtilek, J.; Combes, F.; Salome, P.; Edge, A.; Hamer, S.; Jones, C.; Sun, M.; et al. Molecular Gas in the X-ray Bright Group NGC 5044 as Revealed by ALMA. Astrophys. J. 2014, 792, 94. [CrossRef]

223. Ruffa, I.; Laing, R.A.; Prandoni, I.; Paladino, R.; Parma, P.; Davis, T.A.; Bureau, M. The AGN fuelling/feedback cycle in nearby radio galaxies-III. 3D relative orientations of radio jets and CO discs and their interaction. Mon. Not. R. Astron. Soc. Lett. 2020, 499, 5719-5731. [CrossRef] 
224. Boizelle, B.D.; Walsh, J.L.; Barth, A.J.; Buote, D.A.; Baker, A.J.; Darling, J.; Ho, L.C.; Cohn, J.; Kabasares, K.M. Black Hole Mass Measurements of Radio Galaxies NGC 315 and NGC 4261 Using ALMA CO Observations. arXiv 2020, arXiv:2012.04669.

225. Shulevski, A.; Morganti, R.; Oosterloo, T.; Struve, C. Recurrent radio emission and gas supply: The radio galaxy B2 $0258+35$. Astron. Astrophys. 2012, 545, A91. [CrossRef]

226. Gaspari, M. Shaping the X-ray spectrum of galaxy clusters with AGN feedback and turbulence. Mon. Not. R. Astron. Soc. 2015, 451, L60-L64, [CrossRef]

227. Sutherland, R.S.; Dopita, M.A. Cooling functions for low-density astrophysical plasmas. Astrophys. J. 1993, 88, 253-327. [CrossRef]

228. Mernier, F.; de Plaa, J.; Kaastra, J.S.; Zhang, Y.Y.; Akamatsu, H.; Gu, L.; Kosec, P.; Mao, J.; Pinto, C.; Reiprich, T.H.; et al. Radial metal abundance profiles in the intra-cluster medium of cool-core galaxy clusters, groups, and ellipticals. Astron. Astrophys. 2017, 603, A80, [CrossRef]

229. Pope, E.C.D. Why and when is internally driven AGN feedback energetically favoured? Mon. Not. R. Astron. Soc. 2012, 427, 2-10. [CrossRef]

230. Dalgarno, A.; McCray, R.A. Heating and Ionization of HI Regions. Annu. Rev. Astron. Astrophys. 1972, 10, 375. [CrossRef]

231. Inoue, T.; Inutsuka, S.i. Two-Fluid Magnetohydrodynamic Simulations of Converging H I Flows in the Interstellar Medium. I. Methodology and Basic Results. Astrophys. J. 2008, 687, 303-310. [CrossRef]

232. Hamer, S.L.; Edge, A.C.; Swinbank, A.M.; Wilman, R.J.; Combes, F.; Salomé, P.; Fabian, A.C.; Crawford, C.S.; Russell, H.R.; Hlavacek-Larrondo, J.; et al. Optical emission line nebulae in galaxy cluster cores 1: The morphological, kinematic and spectral properties of the sample. Mon. Not. R. Astron. Soc. 2016, 460, 1758-1789. [CrossRef]

233. Juráňová, A.; Werner, N.; Nulsen, P.E.J.; Gaspari, M.; Lakhchaura, K.; Canning, R.E.A.; Donahue, M.; Hroch, F.; Voit, G.M. Hot gaseous atmospheres of rotating galaxies observed with XMM-Newton. Mon. Not. R. Astron. Soc. 2020, 499, 5163-5174. [CrossRef]

234. Vazza, F.; Brunetti, G.; Gheller, C.; Brunino, R.; Brüggen, M. Massive and refined. II. The statistical properties of turbulent motions in massive galaxy clusters with high spatial resolution. Astron. Astrophys. 2011, 529, A17, [CrossRef]

235. Valentini, M.; Brighenti, F. AGN-stimulated cooling of hot gas in elliptical galaxies. Mon. Not. R. Astron. Soc. 2015, 448, 1979-1998. [CrossRef]

236. Gaspari, M.; Brighenti, F.; Temi, P. Mechanical AGN feedback: Controlling the thermodynamical evolution of elliptical galaxies. Mon. Not. R. Astron. Soc. 2012, 424, 190-209. [CrossRef]

237. Hillel, S.; Soker, N. Gentle Heating by Mixing in Cooling Flow Clusters. Astrophys. J. 2017, 845, 91, [CrossRef]

238. Weinberger, R.; Springel, V.; Pakmor, R.; Nelson, D.; Genel, S.; Pillepich, A.; Vogelsberger, M.; Marinacci, F.; Naiman, J.; Torrey, P.; et al. Supermassive black holes and their feedback effects in the IllustrisTNG simulation. Mon. Not. R. Astron. Soc. 2018, 479, 4056-4072. [CrossRef]

239. Wittor, D.; Gaspari, M. Dissecting the turbulent weather driven by mechanical AGN feedback. Mon. Not. R. Astron. Soc. 2020, 498, 4983-5002. [CrossRef]

240. Sanders, J.S.; Fabian, A.C. Velocity width measurements of the coolest X-ray emitting material in the cores of clusters, groups and elliptical galaxies. Mon. Not. R. Astron. Soc. 2013, 429, 2727-2738. [CrossRef]

241. Ogorzalek, A.; Zhuravleva, I.; Allen, S.W.; Pinto, C.; Werner, N.; Mantz, A.B.; Canning, R.E.A.; Fabian, A.C.; Kaastra, J.S.; de Plaa, J. Improved measurements of turbulence in the hot gaseous atmospheres of nearby giant elliptical galaxies. Mon. Not. R. Astron. Soc. 2017, 472, 1659-1676. [CrossRef]

242. Collaboration, H.; Aharonian, F.; Akamatsu, H.; Akimoto, F.; Allen, S.W.; Angelini, L.; Audard, M.; Awaki, H.; Axelsson, M.; Bamba, A.; et al. Atmospheric gas dynamics in the Perseus cluster observed with Hitomi. Publ. Astron. Soc. Jpn. 2018, 70, 9, [CrossRef]

243. Gaspari, M.; Churazov, E. Constraining turbulence and conduction in the hot ICM through density perturbations. Astron. Astrophys. 2013, 559, A78, [CrossRef]

244. Zhuravleva, I.; Churazov, E.M.; Schekochihin, A.A.; Lau, E.T.; Nagai, D.; Gaspari, M.; Allen, S.W.; Nelson, K.; Parrish, I.J. The Relation between Gas Density and Velocity Power Spectra in Galaxy Clusters: Qualitative Treatment and Cosmological Simulations. Astrophys. J. 2014, 788, L13. [CrossRef]

245. Gaspari, M.; Ruszkowski, M.; Oh, S.P. Chaotic cold accretion on to black holes. Mon. Not. R. Astron. Soc. 2013, 432, 3401-3422. [CrossRef]

246. Voit, G.M. A Role for Turbulence in Circumgalactic Precipitation. Astrophys. J. 2018, 868, 102, [CrossRef]

247. Pizzolato, F.; Soker, N. On the Nature of Feedback Heating in Cooling Flow Clusters. Astrophys. J. 2005, 632, 821-830. [CrossRef]

248. McDonald, M.; Veilleux, S.; Mushotzky, R. The Effect of Environment on the Formation of H $\alpha$ Filaments and Cool Cores in Galaxy Groups and Clusters. Astrophys. J. 2011, 731, 33, [CrossRef]

249. Bondi, H. On spherically symmetrical accretion. Mon. Not. R. Astron. Soc. 1952, 112, 195. [CrossRef]

250. Narayan, R.; Fabian, A.C. Bondi flow from a slowly rotating hot atmosphere. Mon. Not. R. Astron. Soc. 2011, 415, 3721-3730. [CrossRef]

251. Voit, G.M.; Donahue, M.; O'Shea, B.W.; Bryan, G.L.; Sun, M.; Werner, N. Supernova Sweeping and Black Hole Feedback in Elliptical Galaxies. Astrophys. J. 2015, 803, L21, [CrossRef]

252. McNamara, B.R.; Russell, H.R.; Nulsen, P.E.J.; Hogan, M.T.; Fabian, A.C.; Pulido, F.; Edge, A.C. A Mechanism for Stimulating AGN Feedback by Lifting Gas in Massive Galaxies. Astrophys. J. 2016, 830, 79, [CrossRef] 
253. Voit, G.M. A Graphical Interpretation of Circumgalactic Precipitation. Astrophys. J. 2021, 908, L16, [CrossRef]

254. Singh, P.; Voit, G.M.; Nath, B.B. Constraints on precipitation-limited hot halos from massive galaxies to galaxy clusters. Mon. Not. R. Astron. Soc. 2020, [CrossRef]

255. Gaspari, M.; Brighenti, F.; Temi, P. Chaotic cold accretion on to black holes in rotating atmospheres. Astron. Astrophys. 2015, 579, A62, [CrossRef]

256. Caon, N.; Macchetto, D.; Pastoriza, M. A Survey of the Interstellar Medium in Early-Type Galaxies. III. Stellar and Gas Kinematics. Astrophys. J. 2000, 127, 39-58. [CrossRef]

257. Diehl, S.; Statler, T.S. The Hot Interstellar Medium of Normal Elliptical Galaxies. I. A Chandra Gas Gallery and Comparison of X-ray and Optical Morphology. Astrophys. J. 2007, 668, 150-167. [CrossRef]

258. Ulrich, M.H.; Maraschi, L.; Urry, C.M. Variability of Active Galactic Nuclei. Annu. Rev. Astron. Astrophys. 1997, 35, 445-502. [CrossRef]

259. Peterson, B.M. Variability of Active Galactic Nuclei. In Advanced Lectures on the Starburst-AGN; World Scientific ; Aretxaga, I., Kunth, D., Mújica, R., Eds.; Tonantzintla: Puebla, Mexico, 2001; p. 3.

260. Hudson, D.S.; Mittal, R.; Reiprich, T.H.; Nulsen, P.E.J.; Andernach, H.; Sarazin, C.L. What is a cool-core cluster? a detailed analysis of the cores of the X-ray flux-limited HIFLUGCS cluster sample. Astron. Astrophys. 2010, 513, A37, [CrossRef]

261. Ghirardini, V.; Eckert, D.; Ettori, S.; Pointecouteau, E.; Molendi, S.; Gaspari, M.; Rossetti, M.; De Grandi, S.; Roncarelli, M.; Bourdin, H.; et al. Universal thermodynamic properties of the intracluster medium over two decades in radius in the X-COP sample. Astron. Astrophys. 2019, 621, A41, [CrossRef]

262. Gaspari, M.; Sądowski, A. Unifying the Micro and Macro Properties of AGN Feeding and Feedback. Astrophys. J. 2017, 837, 149, [CrossRef]

263. Gaspari, M.; Brighenti, F.; Temi, P.; Ettori, S. Can AGN Feedback Break the Self-similarity of Galaxies, Groups, and Clusters? Astrophys. J. 2014, 783, L10, [CrossRef]

264. Gaspari, M.; Brighenti, F.; D’Ercole, A.; Melioli, C. AGN feedback in galaxy groups: The delicate touch of self-regulated outflows. Mon. Not. R. Astron. Soc. 2011, 415, 1549-1568. [CrossRef]

265. Prasad, D.; Sharma, P.; Babul, A. Cool Core Cycles: Cold Gas and AGN Jet Feedback in Cluster Cores. Astrophys. J. 2015, 811, 108, [CrossRef]

266. Sądowski, A.; Gaspari, M. Kinetic and radiative power from optically thin accretion flows. Mon. Not. R. Astron. Soc. 2017, 468, 1398-1404. [CrossRef]

267. Fukumura, K.; Kazanas, D.; Contopoulos, I.; Behar, E. Magnetohydrodynamic Accretion Disk Winds as X-ray Absorbers in Active Galactic Nuclei. Astrophys. J. 2010, 715, 636-650. [CrossRef]

268. Tombesi, F.; Cappi, M.; Reeves, J.N.; Nemmen, R.S.; Braito, V.; Gaspari, M.; Reynolds, C.S. Unification of X-ray winds in Seyfert galaxies: From ultra-fast outflows to warm absorbers. Mon. Not. R. Astron. Soc. 2013, 430, 1102-1117. [CrossRef]

269. Tchekhovskoy, A.; Narayan, R.; McKinney, J.C. Efficient generation of jets from magnetically arrested accretion on a rapidly spinning black hole. Mon. Not. R. Astron. Soc. 2011, 418, L79-L83, [CrossRef]

270. Russell, H.R.; McNamara, B.R.; Edge, A.C.; Hogan, M.T.; Main, R.A.; Vantyghem, A.N. Radiative efficiency, variability and Bondi accretion on to massive black holes: The transition from radio AGN to quasars in brightest cluster galaxies. Mon. Not. R. Astron. Soc. 2013, 432, 530-553. [CrossRef]

271. Costa, T.; Sijacki, D.; Haehnelt, M.G. Feedback from active galactic nuclei: Energy- versus momentum-driving. Mon. Not. R. Astron. Soc. 2014, 444, 2355-2376. [CrossRef]

272. Giovannini, G. Observational Properties of Jets in Active Galactic Nuclei. Astrophys. Space Sci. 2004, 293, 1-13. [CrossRef]

273. Fiore, F.; Feruglio, C.; Shankar, F.; Bischetti, M.; Bongiorno, A.; Brusa, M.; Carniani, S.; Cicone, C.; Duras, F.; Lamastra, A.; et al. AGN wind scaling relations and the co-evolution of black holes and galaxies. Astron. Astrophys. 2017, 601, A143, [CrossRef]

274. Brighenti, F.; Mathews, W.G.; Temi, P. Hot Gaseous Atmospheres in Galaxy Groups and Clusters Are Both Heated and Cooled by X-ray Cavities. Astrophys. J. 2015, 802, 118, [CrossRef]

275. Gaspari, M.; Churazov, E.; Nagai, D.; Lau, E.T.; Zhuravleva, I. The relation between gas density and velocity power spectra in galaxy clusters: High-resolution hydrodynamic simulations and the role of conduction. Astron. Astrophys. 2014, 569, A67, [CrossRef]

276. Fabian, A.C.; Walker, S.A.; Russell, H.R.; Pinto, C.; Sanders, J.S.; Reynolds, C.S. Do sound waves transport the AGN energy in the Perseus cluster? Mon. Not. R. Astron. Soc. 2017, 464, L1-L5, [CrossRef]

277. Cielo, S.; Babul, A.; Antonuccio-Delogu, V.; Silk, J.; Volonteri, M. Feedback from reorienting AGN jets. I. Jet-ICM coupling, cavity properties and global energetics. Astron. Astrophys. 2018, 617, A58. [CrossRef]

278. Voit, G.M.; Bryan, G.L.; Prasad, D.; Frisbie, R.; Li, Y.; Donahue, M.; O’Shea, B.W.; Sun, M.; Werner, N. A Black Hole Feedback Valve in Massive Galaxies. Astrophys. J. 2020, 899, 70, [CrossRef]

279. Goulding, A.D.; Greene, J.E.; Ma, C.P.; Veale, M.; Bogdan, A.; Nyland, K.; Blakeslee, J.P.; McConnell, N.J.; Thomas, J. The MASSIVE Survey. IV. The X-ray Halos of the Most Massive Early-type Galaxies in the Nearby Universe. Astrophys. J. 2016, 826, 167, [CrossRef]

280. Tremmel, M.; Karcher, M.; Governato, F.; Volonteri, M.; Quinn, T.R.; Pontzen, A.; Anderson, L.; Bellovary, J. The Romulus cosmological simulations: A physical approach to the formation, dynamics and accretion models of SMBHs. Mon. Not. R. Astron. Soc. 2017, 470, 1121-1139. [CrossRef] 
281. Dubois, Y.; Beckmann, R.; Bournaud, F.; Choi, H.; Devriendt, J.; Jackson, R.; Kaviraj, S.; Kimm, T.; Kraljic, K.; Laigle, C.; et al. Introducing the NewHorizon simulation: Galaxy properties with resolved internal dynamics across cosmic time. arXiv 2020, arXiv:2009.10578.

282. Henden, N.A.; Puchwein, E.; Shen, S.; Sijacki, D. The FABLE simulations: A feedback model for galaxies, groups, and clusters. Mon. Not. R. Astron. Soc. 2018, 479, 5385-5412. [CrossRef]

283. Dubois, Y.; Pichon, C.; Welker, C.; Le Borgne, D.; Devriendt, J.; Laigle, C.; Codis, S.; Pogosyan, D.; Arnouts, S.; Benabed, K.; et al. Dancing in the dark: Galactic properties trace spin swings along the cosmic web. Mon. Not. R. Astron. Soc. 2014, 444, 1453-1468. [CrossRef]

284. Schaye, J.; Crain, R.A.; Bower, R.G.; Furlong, M.; Schaller, M.; Theuns, T.; Dalla Vecchia, C.; Frenk, C.S.; McCarthy, I.G.; Helly, J.C.; et al. The EAGLE project: Simulating the evolution and assembly of galaxies and their environments. Mon. Not. R. Astron. Soc. 2015, 446, 521-554. [CrossRef]

285. Vogelsberger, M.; Genel, S.; Springel, V.; Torrey, P.; Sijacki, D.; Xu, D.; Snyder, G.; Nelson, D.; Hernquist, L. Introducing the Illustris Project: Simulating the coevolution of dark and visible matter in the Universe. Mon. Not. R. Astron. Soc. 2014, 444, 1518-1547. [CrossRef]

286. Springel, V.; Pakmor, R.; Pillepich, A.; Weinberger, R.; Nelson, D.; Hernquist, L.; Vogelsberger, M.; Genel, S.; Torrey, P.; Marinacci, F.; et al. First results from the IllustrisTNG simulations: Matter and galaxy clustering. Mon. Not. R. Astron. Soc. 2018, 475, 676-698. [CrossRef]

287. Davé, R.; Anglés-Alcázar, D.; Narayanan, D.; Li, Q.; Rafieferantsoa, M.H.; Appleby, S. SIMBA: Cosmological simulations with black hole growth and feedback. Mon. Not. R. Astron. Soc. 2019, 486, 2827-2849. [CrossRef]

288. Robson, D.; Davé, R. X-ray emission from hot gas in galaxy groups and clusters in SIMBA. Mon. Not. R. Astron. Soc. 2020, 498, 3061-3076. [CrossRef]

289. Khandai, N.; Di Matteo, T.; Croft, R.; Wilkins, S.; Feng, Y.; Tucker, E.; DeGraf, C.; Liu, M.S. The MassiveBlack-II simulation: The evolution of haloes and galaxies to $\mathrm{z} \sim 0$. Mon. Not. R. Astron. Soc. 2015, 450, 1349-1374. [CrossRef]

290. Hirschmann, M.; Dolag, K.; Saro, A.; Bachmann, L.; Borgani, S.; Burkert, A. Cosmological simulations of black hole growth: AGN luminosities and downsizing. Mon. Not. R. Astron. Soc. 2014, 442, 2304-2324. [CrossRef]

291. Lee, J.; Shin, J.; Snaith, O.N.; Kim, Y.; Few, C.G.; Devriendt, J.; Dubois, Y.; Cox, L.M.; Hong, S.E.; Kwon, O.K.; et al. The Horizon Run 5 Cosmological Hydrodynamical Simulation: Probing Galaxy Formation from Kilo- to Gigaparsec Scales. Astrophys. J. 2021, 908, 11, [CrossRef]

292. Springel, V.; Di Matteo, T.; Hernquist, L. Modelling feedback from stars and black holes in galaxy mergers. Mon. Not. R. Astron. Soc. 2005, 361, 776-794. [CrossRef]

293. Springel, V. E pur si muove: Galilean-invariant cosmological hydrodynamical simulations on a moving mesh. Mon. Not. R. Astron. Soc. 2010, 401, 791-851. [CrossRef]

294. Weinberger, R.; Springel, V.; Pakmor, R. The AREPO Public Code Release. Astrophys. J. 2020, 248, 32, [CrossRef]

295. Hopkins, P.F. A new class of accurate, mesh-free hydrodynamic simulation methods. Mon. Not. R. Astron. Soc. 2015, 450, 53-110. [CrossRef]

296. Teyssier, R. Cosmological hydrodynamics with adaptive mesh refinement. A new high resolution code called RAMSES. Astron. Astrophys. 2002, 385, 337-364. [CrossRef]

297. Menon, H.; Wesolowski, L.; Zheng, G.; Jetley, P.; Kale, L.; Quinn, T.; Governato, F. Adaptive techniques for clustered N-body cosmological simulations. Comput. Astrophys. Cosmol. 2015, 2, 1, [CrossRef]

298. Booth, C.M.; Schaye, J. Cosmological simulations of the growth of supermassive black holes and feedback from active galactic nuclei: Method and tests. Mon. Not. R. Astron. Soc. 2009, 398, 53-74. [CrossRef]

299. Hoyle, F.; Lyttleton, R.A. The effect of interstellar matter on climatic variation. Proc. Camb. Philos. Soc. 1939, 35, 405. [CrossRef]

300. Bondi, H.; Hoyle, F. On the mechanism of accretion by stars. Mon. Not. R. Astron. Soc. 1944, 104, 273. [CrossRef]

301. Di Matteo, T.; Springel, V.; Hernquist, L. Energy input from quasars regulates the growth and activity of black holes and their host galaxies. Nature 2005, 433, 604-607. [CrossRef]

302. Sijacki, D.; Springel, V.; Di Matteo, T.; Hernquist, L. A unified model for AGN feedback in cosmological simulations of structure formation. Mon. Not. R. Astron. Soc. 2007, 380, 877-900. [CrossRef]

303. Bhattacharya, S.; Di Matteo, T.; Kosowsky, A. Effects of quasar feedback in galaxy groups. Mon. Not. R. Astron. Soc. 2008, 389, 34-44. [CrossRef]

304. Schaye, J.; Dalla Vecchia, C.; Booth, C.M.; Wiersma, R.P.C.; Theuns, T.; Haas, M.R.; Bertone, S.; Duffy, A.R.; McCarthy, I.G.; van de Voort, F. The physics driving the cosmic star formation history. Mon. Not. R. Astron. Soc. 2010, 402, 1536-1560. [CrossRef]

305. Shakura, N.I.; Sunyaev, R.A. Reprint of 1973A\&A....24..337S. Black holes in binary systems. Observational appearance. Astron. Astrophys. 1973, 500, 33-51.

306. Rosas-Guevara, Y.M.; Bower, R.G.; Schaye, J.; Furlong, M.; Frenk, C.S.; Booth, C.M.; Crain, R.A.; Dalla Vecchia, C.; Schaller, M.; Theuns, T. The impact of angular momentum on black hole accretion rates in simulations of galaxy formation. Mon. Not. R. Astron. Soc. 2015, 454, 1038-1057. [CrossRef]

307. Vogelsberger, M.; Genel, S.; Sijacki, D.; Torrey, P.; Springel, V.; Hernquist, L. A model for cosmological simulations of galaxy formation physics. Mon. Not. R. Astron. Soc. 2013, 436, 3031-3067. [CrossRef] 
308. Weinberger, R.; Springel, V.; Hernquist, L.; Pillepich, A.; Marinacci, F.; Pakmor, R.; Nelson, D.; Genel, S.; Vogelsberger, M.; Naiman, J.; et al. Simulating galaxy formation with black hole driven thermal and kinetic feedback. Mon. Not. R. Astron. Soc. 2017, 465, 3291-3308. [CrossRef]

309. Pillepich, A.; Springel, V.; Nelson, D.; Genel, S.; Naiman, J.; Pakmor, R.; Hernquist, L.; Torrey, P.; Vogelsberger, M.; Weinberger, R.; et al. Simulating galaxy formation with the IllustrisTNG model. Mon. Not. R. Astron. Soc. 2018, 473, 4077-4106. [CrossRef]

310. Dubois, Y.; Devriendt, J.; Slyz, A.; Teyssier, R. Self-regulated growth of supermassive black holes by a dual jet-heating active galactic nucleus feedback mechanism: Methods, tests and implications for cosmological simulations. Mon. Not. R. Astron. Soc. 2012, 420, 2662-2683. [CrossRef]

311. Beck, A.M.; Murante, G.; Arth, A.; Remus, R.S.; Teklu, A.F.; Donnert, J.M.F.; Planelles, S.; Beck, M.C.; Förster, P.; Imgrund, M.; et al. An improved SPH scheme for cosmological simulations. Mon. Not. R. Astron. Soc. 2016, 455, 2110-2130. [CrossRef]

312. Sembolini, F.; Yepes, G.; De Petris, M.; Gottlöber, S.; Lamagna, L.; Comis, B. The MUSIC of galaxy clusters-I. Baryon properties and scaling relations of the thermal Sunyaev-Zel'dovich effect. Mon. Not. R. Astron. Soc. 2013, 429, 323-343. [CrossRef]

313. Cui, W.; Knebe, A.; Yepes, G.; Pearce, F.; Power, C.; Dave, R.; Arth, A.; Borgani, S.; Dolag, K.; Elahi, P.; et al. The Three Hundred project: A large catalogue of theoretically modelled galaxy clusters for cosmological and astrophysical applications. Mon. Not. R. Astron. Soc. 2018, 480, 2898-2915. [CrossRef]

314. Rasia, E.; Borgani, S.; Murante, G.; Planelles, S.; Beck, A.M.; Biffi, V.; Ragone-Figueroa, C.; Granato, G.L.; Steinborn, L.K.; Dolag, K. Cool Core Clusters from Cosmological Simulations. Astrophys. J. 2015, 813, L17, [CrossRef]

315. McCarthy, I.G.; Schaye, J.; Ponman, T.J.; Bower, R.G.; Booth, C.M.; Dalla Vecchia, C.; Crain, R.A.; Springel, V.; Theuns, T.; Wiersma, R.P.C. The case for AGN feedback in galaxy groups. Mon. Not. R. Astron. Soc. 2010, 406, 822-839. [CrossRef]

316. Steinborn, L.K.; Dolag, K.; Hirschmann, M.; Prieto, M.A.; Remus, R.S. A refined sub-grid model for black hole accretion and AGN feedback in large cosmological simulations. Mon. Not. R. Astron. Soc. 2015, 448, 1504-1525. [CrossRef]

317. Barnes, D.J.; Kay, S.T.; Bahé, Y.M.; Dalla Vecchia, C.; McCarthy, I.G.; Schaye, J.; Bower, R.G.; Jenkins, A.; Thomas, P.A.; Schaller, M.; et al. The Cluster-EAGLE project: Global properties of simulated clusters with resolved galaxies. Mon. Not. R. Astron. Soc. 2017, 471, 1088-1106. [CrossRef]

318. Bahé, Y.M.; Barnes, D.J.; Dalla Vecchia, C.; Kay, S.T.; White, S.D.M.; McCarthy, I.G.; Schaye, J.; Bower, R.G.; Crain, R.A.; Theuns, T.; et al. The Hydrangea simulations: Galaxy formation in and around massive clusters. Mon. Not. R. Astron. Soc. 2017, 470, 4186-4208. [CrossRef]

319. Bassini, L.; Rasia, E.; Borgani, S.; Ragone-Figueroa, C.; Biffi, V.; Dolag, K.; Gaspari, M.; Granato, G.L.; Murante, G.; Taffoni, G.; et al. Black hole mass and cluster mass correlation in cosmological hydro-dynamical simulations. arXiv 2019, arXiv:1903.03142.

320. Martín-Navarro, I.; Burchett, J.N.; Mezcua, M. Black hole feedback and the evolution of massive early-type galaxies. Mon. Not. R. Astron. Soc. 2020, 491, 1311-1319. [CrossRef]

321. Truong, N.; Pillepich, A.; Werner, N. Correlations between supermassive black holes and hot gas atmospheres in IllustrisTNG and X-ray observations. Mon. Not. R. Astron. Soc. 2021, 501, 2210-2230. [CrossRef]

322. van Daalen, M.P.; Schaye, J.; Booth, C.M.; Dalla Vecchia, C. The effects of galaxy formation on the matter power spectrum: A challenge for precision cosmology. Mon. Not. R. Astron. Soc. 2011, 415, 3649-3665. [CrossRef]

323. Semboloni, E.; Hoekstra, H.; Schaye, J.; van Daalen, M.P.; McCarthy, I.G. Quantifying the effect of baryon physics on weak lensing tomography. Mon. Not. R. Astron. Soc. 2011, 417, 2020-2035. [CrossRef]

324. Chisari, N.E.; Mead, A.J.; Joudaki, S.; Ferreira, P.G.; Schneider, A.; Mohr, J.; Tröster, T.; Alonso, D.; McCarthy, I.G.; Martin-Alvarez, S.; et al. Modelling baryonic feedback for survey cosmology. Open J. Astrophys. 2019, 2, 4, [CrossRef]

325. Amendola, L.; Appleby, S.; Avgoustidis, A.; Bacon, D.; Baker, T.; Baldi, M.; Bartolo, N.; Blanchard, A.; Bonvin, C.; Borgani, S.; et al. Cosmology and fundamental physics with the Euclid satellite. Living Rev. Relativ. 2018, 21, 2, [CrossRef] [PubMed]

326. Schneider, A.; Stoira, N.; Refregier, A.; Weiss, A.J.; Knabenhans, M.; Stadel, J.; Teyssier, R. Baryonic effects for weak lensing. Part I. Power spectrum and covariance matrix. J. Cosmol. Astropart. Phys. 2020, 2020, 19, [CrossRef]

327. Schneider, A.; Refregier, A.; Grandis, S.; Eckert, D.; Stoira, N.; Kacprzak, T.; Knabenhans, M.; Stadel, J.; Teyssier, R. Baryonic effects for weak lensing. Part II. Combination with X-ray data and extended cosmologies. J. Cosmol. Astropart. Phys. 2020, 2020, 20, [CrossRef]

328. van Daalen, M.P.; McCarthy, I.G.; Schaye, J. Exploring the effects of galaxy formation on matter clustering through a library of simulation power spectra. Mon. Not. R. Astron. Soc. 2020, 491, 2424-2446. [CrossRef]

329. Schneider, A.; Teyssier, R.; Stadel, J.; Chisari, N.E.; Le Brun, A.M.C.; Amara, A.; Refregier, A. Quantifying baryon effects on the matter power spectrum and the weak lensing shear correlation. J. Cosmol. Astropart. Phys. 2019, 2019, 020, [CrossRef]

330. Battaglia, N.; Bond, J.R.; Pfrommer, C.; Sievers, J.L. On the Cluster Physics of Sunyaev-Zel'dovich and X-ray Surveys. II. Deconstructing the Thermal SZ Power Spectrum. Astrophys. J. 2012, 758, 75, [CrossRef]

331. McCarthy, I.G.; Le Brun, A.M.C.; Schaye, J.; Holder, G.P. The thermal Sunyaev-Zel'dovich effect power spectrum in light of Planck. Mon. Not. R. Astron. Soc. 2014, 440, 3645-3657. [CrossRef]

332. McCarthy, I.G.; Bird, S.; Schaye, J.; Harnois-Deraps, J.; Font, A.S.; van Waerbeke, L. The BAHAMAS project: The CMB-large-scale structure tension and the roles of massive neutrinos and galaxy formation. Mon. Not. R. Astron. Soc. 2018, 476, 2999-3030. [CrossRef]

333. Ramos-Ceja, M.E.; Basu, K.; Pacaud, F.; Bertoldi, F. Constraining the intracluster pressure profile from the thermal SZ power spectrum. Astron. Astrophys. 2015, 583, A111, [CrossRef] 
334. Arnaud, M.; Pratt, G.W.; Piffaretti, R.; Böhringer, H.; Croston, J.H.; Pointecouteau, E. The universal galaxy cluster pressure profile from a representative sample of nearby systems (REXCESS) and the $\mathrm{Y}_{S Z}-\mathrm{M}_{500}$ relation. Astron. Astrophys. 2010, 517, A92, [CrossRef]

335. Battaglia, N.; Hill, J.C.; Murray, N. Deconstructing Thermal Sunyaev-Zel'dovich-Gravitational Lensing Cross-correlations: Implications for the Intracluster Medium. Astrophys. J. 2015, 812, 154, [CrossRef]

336. Hojjati, A.; McCarthy, I.G.; Harnois-Deraps, J.; Ma, Y.Z.; Van Waerbeke, L.; Hinshaw, G.; Le Brun, A.M.C. Dissecting the thermal Sunyaev-Zeldovich-gravitational lensing cross-correlation with hydrodynamical simulations. J. Cosmol. Astropart. Phys. 2015, 2015, 047, [CrossRef]

337. Velliscig, M.; van Daalen, M.P.; Schaye, J.; McCarthy, I.G.; Cacciato, M.; Le Brun, A.M.C.; Dalla Vecchia, C. The impact of galaxy formation on the total mass, mass profile and abundance of haloes. Mon. Not. R. Astron. Soc. 2014, 442, 2641-2658. [CrossRef]

338. Cui, W.; Borgani, S.; Murante, G. The effect of active galactic nuclei feedback on the halo mass function. Mon. Not. R. Astron. Soc. 2014, 441, 1769-1782. [CrossRef]

339. Bocquet, S.; Saro, A.; Dolag, K.; Mohr, J.J. Halo mass function: Baryon impact, fitting formulae, and implications for cluster cosmology. Mon. Not. R. Astron. Soc. 2016, 456, 2361-2373. [CrossRef]

340. Schaller, M.; Frenk, C.S.; Bower, R.G.; Theuns, T.; Jenkins, A.; Schaye, J.; Crain, R.A.; Furlong, M.; Dalla Vecchia, C.; McCarthy, I.G. Baryon effects on the internal structure of $\Lambda \mathrm{CDM}$ haloes in the EAGLE simulations. Mon. Not. R. Astron. Soc. 2015, 451, 1247-1267. [CrossRef]

341. Xu, W.; Ramos-Ceja, M.E.; Pacaud, F.; Reiprich, T.H.; Erben, T. A new X-ray-selected sample of very extended galaxy groups from the ROSAT All-Sky Survey. Astron. Astrophys. 2018, 619, A162, [CrossRef]

342. Merloni, A.; Predehl, P.; Becker, W.; Böhringer, H.; Boller, T.; Brunner, H.; Brusa, M.; Dennerl, K.; Freyberg, M.; Friedrich, P.; et al. eROSITA Science Book: Mapping the Structure of the Energetic Universe. arXiv 2012, arXiv:1209.3114.

343. Käfer, F.; Finoguenov, A.; Eckert, D.; Clerc, N.; Ramos-Ceja, M.E.; Sanders, J.S.; Ghirardini, V. Toward the low-scatter selection of X-ray clusters. Galaxy cluster detection with eROSITA through cluster outskirts. Astron. Astrophys. 2020, 634, A8, [CrossRef]

344. Freyberg, M.; Perinati, E.; Pacaud, F.; Eraerds, T.; Churazov, E.; Dennerl, K.; Predehl, P.; Merloni, A.; Meidinger, N.; Bulbul, E.; et al. SRG/eROSITA in-flight background at L2. In Proceedings of the SPIE Digital Library, SPIE Astronomical Telescopes + Instrumentation, Online Conference, 13-18 December 2020; Society of Photo-Optical Instrumentation Engineers (SPIE) Conference Series; 2020; Volume 11444, p. 114441O. [CrossRef]

345. Böhringer, H.; Chon, G.; Collins, C.A.; Guzzo, L.; Nowak, N.; Bobrovskyi, S. The extended ROSAT-ESO flux limited X-ray galaxy cluster survey (REFLEX II) II. Construction and properties of the survey. Astron. Astrophys. 2013, 555, A30, [CrossRef]

346. Planck Collaboration XXVII. Planck 2015 results. XXVII. The second Planck catalogue of Sunyaev-Zeldovich sources. Astron. Astrophys. 2016, 594, A27, [CrossRef]

347. Benson, B.A.; Ade, P.A.R.; Ahmed, Z.; Allen, S.W.; Arnold, K.; Austermann, J.E.; Bender, A.N.; Bleem, L.E.; Carlstrom, J.E.; Chang, C.L.; et al. SPT-3G: A next-generation cosmic microwave background polarization experiment on the South Pole telescope. In Proceedings of the SPIE Astronomical Telescopes + Instrumentation, Montréal, QC, Canada, 22-27 June 2014; Volume 9153, p. 91531P. [CrossRef]

348. XRISM Science Team. Science with the X-ray Imaging and Spectroscopy Mission (XRISM). arXiv 2020, arXiv:2003.04962.

349. Pinto, C.; Sanders, J.S.; Werner, N.; de Plaa, J.; Fabian, A.C.; Zhang, Y.Y.; Kaastra, J.S.; Finoguenov, A.; Ahoranta, J. Chemical Enrichment RGS cluster Sample (CHEERS): Constraints on turbulence. Astron. Astrophys. 2015, 575, A38, [CrossRef]

350. Ahoranta, J.; Finoguenov, A.; Pinto, C.; Sanders, J.; Kaastra, J.; de Plaa, J.; Fabian, A. Observations of asymmetric velocity fields and gas cooling in the NGC 4636 galaxy group X-ray halo. Astron. Astrophys. 2016, 592, A145. [CrossRef]

351. Hitomi Collaboration. The quiescent intracluster medium in the core of the Perseus cluster. Nature 2016, 535, 117-121. [CrossRef] [PubMed]

352. Barret, D.; Decourchelle, A.; Fabian, A.; Guainazzi, M.; Nandra, K.; Smith, R.; den Herder, J.W. The Athena space X-ray observatory and the astrophysics of hot plasma. Astron. Nachrichten 2020, 341, 224-235. [CrossRef]

353. Zhang, C.; Ramos-Ceja, M.E.; Pacaud, F.; Reiprich, T.H. High-redshift galaxy groups as seen by ATHENA/WFI. Astron. Astrophys. 2020, 642, A17. [CrossRef]

354. Croston, J.H.; Sanders, J.S.; Heinz, S.; Hardcastle, M.J.; Zhuravleva, I.; Bîrzan, L.; Bower, R.G.; Brüggen, M.; Churazov, E.; Edge, A.C.; et al. The Hot and Energetic Universe: AGN feedback in galaxy clusters and groups. arXiv 2013, arXiv:1306.2323.

355. Gaskin, J.A.; Swartz, D.A.; Vikhlinin, A.; Özel, F.; Gelmis, K.E.; Arenberg, J.W.; Bandler, S.R.; Bautz, M.W.; Civitani, M.M.; Dominguez, A.; et al. Lynx X-ray Observatory: An overview. J. Astron. Telesc. Instruments, Syst. 2019, 5, 021001. [CrossRef]

356. Hurley-Walker, N.; Callingham, J.R.; Hancock, P.J.; Franzen, T.M.O.; Hindson, L.; Kapińska, A.D.; Morgan, J.; Offringa, A.R.; Wayth, R.B.; Wu, C.; et al. GaLactic and Extragalactic All-sky Murchison Widefield Array (GLEAM) survey-I. A low-frequency extragalactic catalogue. Mon. Not. R. Astron. Soc. Lett. 2017, 464, 1146-1167. [CrossRef]

357. McConnell, D.; Hale, C.L.; Lenc, E.; Banfield, J.K.; Heald, G.; Hotan, A.W.; Leung, J.K.; Moss, V.A.; Murphy, T.; O’Brien, A.; et al. The Rapid ASKAP Continuum Survey I: Design and first results. Publ. Astron. Soc. Aust. 2020, 37, e048. [CrossRef]

358. Koribalski, B.S.; Staveley-Smith, L.; Westmeier, T.; Serra, P.; Spekkens, K.; Wong, O.I.; Lee-Waddell, K.; Lagos, C.D.P.; Obreschkow, D.; Ryan-Weber, E.V.; et al. WALLABY_An SKA Pathfinder HI survey. Astrophys. Space Sci. 2020, 365, 118. [CrossRef] 
359. Jarvis, M.; Taylor, R.; Agudo, I.; Allison, J.R.; Deane, R.P.; Frank, B.; Gupta, N.; Heywood, I.; Maddox, N.; McAlpine, K.; et al. The MeerKAT International GHz Tiered Extragalactic Exploration (MIGHTEE) Survey. In Proceedings of the 2016 MeerKAT Science: On the Pathway to the SKA, MeerKAT, Stellenbosch, South Africa, 25-27 May 2016; p. 6.

360. Oosterloo, T.A.; Zhang, M.L.; Lucero, D.M.; Carignan, C. Galaxy interactions in loose galaxy groups: KAT-7 and VLA HI Observations of the IC 1459 group. arXiv 2018, arXiv:1803.08263.

361. For, B.Q.; Staveley-Smith, L.; Westmeier, T.; Whiting, M.; Oh, S.H.; Koribalski, B.; Wang, J.; Wong, O.I.; Bekiaris, G.; Cortese, L.; et al. WALLABY early science-V. ASKAP H I imaging of the Lyon Group of Galaxies 351. Mon. Not. R. Astron. Soc. Lett. 2019, 489, 5723-5741. [CrossRef]

362. Braun, R.; Bonaldi, A.; Bourke, T.; Keane, E.; Wagg, J. Anticipated Performance of the Square Kilometre Array-Phase 1 (SKA1). arXiv 2019, arXiv:1912.12699.

363. Prandoni, I.; Seymour, N. Revealing the Physics and Evolution of Galaxies and Galaxy Clusters with SKA Continuum Surveys. In Proceedings of theAdvancing Astrophysics with the Square Kilometre Array (AASKA14), Giardini Naxos, Italy, 8-13 June 2015; p. 67,

364. McAlpine, K.; Prandoni, I.; Jarvis, M.; Seymour, N.; Padovani, P.; Best, P.; Simpson, C.; Guidetti, D.; Murphy, E.; Huynh, M.; et al. The SKA view of the Interplay between SF and AGN Activity and its role in Galaxy Evolution. In Proceedings of the Advancing Astrophysics with the Square Kilometre Array (AASKA14), Giardini Naxos, Italy, 8-13 June 2015; p. 83.

365. Heald, G.; Mao, S.; Vacca, V.; Akahori, T.; Damas-Segovia, A.; Gaensler, B.; Hoeft, M.; Agudo, I.; Basu, A.; Beck, R.; et al. Magnetism Science with the Square Kilometre Array. Galaxies 2020, 8, 53, [CrossRef]

366. Kale, R.; Dwarakanath, K.S.; Vir Lal, D.; Bagchi, J.; Paul, S.; Malu, S.; Datta, A.; Parekh, V.; Sharma, P.; Pandey-Pommier, M. Clusters of Galaxies and the Cosmic Web with Square Kilometre Array. J. Astrophys. Astron. 2016, 37, 31, [CrossRef]

367. Stacey, G.J.; Aravena, M.; Basu, K.; Battaglia, N.; Beringue, B.; Bertoldi, F.; Bond, J.R.; Breysse, P.; Bustos, R.; Chapman, S.; et al. CCAT-Prime: Science with an ultra-widefield submillimeter observatory on Cerro Chajnantor. In Proceedings of the SPIE Astronomical Telescopes + Instrumentation, Austin, TX, USA, 12-14 June 2018; Volume 10700, p. 107001M. [CrossRef]

368. Lee, A.; Abitbol, M.H.; Adachi, S.; Ade, P.; Aguirre, J.; Ahmed, Z.; Aiola, S.; Ali, A.; Alonso, D.; Alvarez, M.A.; et al. The Simons Observatory. Bull. Am. Astron. Soc. 2019, 51, 147.

369. Abazajian, K.N.; Adshead, P.; Ahmed, Z.; Allen, S.W.; Alonso, D.; Arnold, K.S.; Baccigalupi, C.; Bartlett, J.G.; Battaglia, N.; Benson, B.A.; et al. CMB-S4 Science Book, First Edition. arXiv 2016, arXiv:1610.02743.

370. Planck Collaboration; Ade, P.A.R.; Aghanim, N.; Arnaud, M.; Ashdown, M.; Atrio-Barandela, F.; Aumont, J.; Baccigalupi, C.; Balbi, A.; Banday, A.J.; et al. Planck intermediate results. XI. The gas content of dark matter halos: The Sunyaev-Zeldovich-stellar mass relation for locally brightest galaxies. Astron. Astrophys. 2013, 557, A52, [CrossRef]

371. Le Brun, A.M.C.; McCarthy, I.G.; Melin, J.B. Testing Sunyaev-Zel'dovich measurements of the hot gas content of dark matter haloes using synthetic skies. Mon. Not. R. Astron. Soc. 2015, 451, 3868-3881. [CrossRef]

372. Klaassen, P.D.; Mroczkowski, T.K.; Cicone, C.; Hatziminaoglou, E.; Sartori, S.; De Breuck, C.; Bryan, S.; Dicker, S.R.; Duran, C.; Groppi, C.; et al. The Atacama Large Aperture Submillimeter Telescope (AtLAST).In Proceedings of the SPIE Astronomical Telescopes + Instrumentation, Online Conference, 13-18 December 2020; Society of Photo-Optical Instrumentation Engineers (SPIE) Conference Series; Volume 11445, p. 114452F, [CrossRef]

373. Mroczkowski, T.; Nagai, D.; Basu, K.; Chluba, J.; Sayers, J.; Adam, R.; Churazov, E.; Crites, A.; Di Mascolo, L.; Eckert, D.; et al Astrophysics with the Spatially and Spectrally Resolved Sunyaev-Zeldovich Effects. A Millimetre/Submillimetre Probe of the Warm and Hot Universe. Space Sci. Rev. 2019, 215, 17, [CrossRef]

374. Hill, J.C.; Ferraro, S.; Battaglia, N.; Liu, J.; Spergel, D.N. Kinematic Sunyaev-Zel'dovich Effect with Projected Fields: A Novel Probe of the Baryon Distribution with Planck, WMAP, and WISE Data. Phys. Rev. Lett. 2016, 117, 051301, [CrossRef] [PubMed]

375. Ferraro, S.; Hill, J.C.; Battaglia, N.; Liu, J.; Spergel, D.N. Kinematic Sunyaev-Zel'dovich effect with projected fields. II. Prospects, challenges, and comparison with simulations. Phys. Rev. D 2016, 94, 123526, [CrossRef]

376. De Bernardis, F.; Aiola, S.; Vavagiakis, E.M.; Battaglia, N.; Niemack, M.D.; Beall, J.; Becker, D.T.; Bond, J.R.; Calabrese, E.; Cho, H.; et al. Detection of the pairwise kinematic Sunyaev-Zel'dovich effect with BOSS DR11 and the Atacama Cosmology Telescope. J. Cosmol. Astropart. Phys. 2017, 2017, 008, [CrossRef]

377. Calafut, V.; Gallardo, P.A.; Vavagiakis, E.M.; Amodeo, S.; Aiola, S.; Austermann, J.E.; Battaglia, N.; Battistelli, E.S.; Beall, J.A.; Bean, R.; et al. The Atacama Cosmology Telescope: Detection of the Pairwise Kinematic Sunyaev-Zel'dovich Effect with SDSS DR15 Galaxies. arXiv 2021, arXiv:2101.08374.

378. Planck Collaboration; Ade, P.A.R.; Aghanim, N.; Arnaud, M.; Ashdown, M.; Aubourg, E.; Aumont, J.; Baccigalupi, C.; Banday, A.J.; Barreiro, R.B.; et al. Planck intermediate results. XXXVII. Evidence of unbound gas from the kinetic Sunyaev-Zeldovich effect. Astron. Astrophys. 2016, 586, A140, [CrossRef]

379. Battaglia, N.; Ferraro, S.; Schaan, E.; Spergel, D.N. Future constraints on halo thermodynamics from combined Sunyaev-Zel'dovich measurements. J. Cosmol. Astropart. Phys. 2017, 2017, 040, [CrossRef]

380. Chaves-Montero, J.; Hernandez-Monteagudo, C.; Angulo, R.E.; Emberson, J.D. Measuring the evolution of intergalactic gas from $\mathrm{z}=0$ to 5 using the kinematic Sunyaev-Zel'dovich effect. arXiv 2019, arXiv:1911.10690.

381. Rafferty, D.A.; McNamara, B.R.; Nulsen, P.E.J.; Wise, M.W. The Feedback-regulated Growth of Black Holes and Bulges through Gas Accretion and Starbursts in Cluster Central Dominant Galaxies. Astrophys. J. 2006, 652, 216-231. [CrossRef] 
382. Lal, D.V.; Kraft, R.P.; Randall, S.W.; Forman, W.R.; Nulsen, P.E.J.; Roediger, E.; ZuHone, J.A.; Hardcastle, M.J.; Jones, C.; Croston, J.H. Gas Sloshing and Radio Galaxy Dynamics in the Core of the 3C 449 Group. Astrophys. J. 2013, 764, 83, [CrossRef]

383. Pandge, M.B.; Sonkamble, S.S.; Parekh, V.; Dabhade, P.; Parmar, A.; Patil, M.K.; Raychaudhury, S. AGN Feedback in Galaxy Groups: A Detailed Study of X-ray Features and Diffuse Radio Emission in IC 1262. Astrophys. J. 2019, 870, 62, [CrossRef]

384. Randall, S.W.; Forman, W.R.; Giacintucci, S.; Nulsen, P.E.J.; Sun, M.; Jones, C.; Churazov, E.; David, L.P.; Kraft, R.; Donahue, M.; et al. Shocks and Cavities from Multiple Outbursts in the Galaxy Group NGC 5813: A Window to Active Galactic Nucleus Feedback. Astrophys. J. 2011, 726, 86. [CrossRef]

385. Schellenberger, G.; Vrtilek, J.M.; David, L.; O’Sullivan, E.; Giacintucci, S.; Johnston-Hollitt, M.; Duchesne, S.W.; Raychaudhury, S. NGC 741-Mergers and AGN Feedback on a Galaxy-group Scale. Astrophys. J. 2017, 845, 84, [CrossRef]

386. Machacek, M.E.; Kraft, R.P.; Jones, C.; Forman, W.R.; Hardcastle, M.J. X-ray Constraints on Galaxy-Gas-Jet Interactions in the Dumbbell Galaxies NGC 4782 and NGC 4783 in the LGG 316 Galaxy Group. Astrophys. J. 2007, 664, 804-819. [CrossRef]

387. Randall, S.W.; Jones, C.; Markevitch, M.; Blanton, E.L.; Nulsen, P.E.J.; Forman, W.R. Gas Sloshing and Bubbles in the Galaxy Group NGC 5098. Astrophys. J. 2009, 700, 1404-1414. [CrossRef]

388. O'Sullivan, E.; Kolokythas, K.; Kantharia, N.G.; Raychaudhury, S.; David, L.P.; Vrtilek, J.M. The origin of the X-ray, radio and H I structures in the NGC 5903 galaxy group. Mon. Not. R. Astron. Soc. Lett. 2018, 473, 5248. [CrossRef]

389. Machacek, M.E.; O'Sullivan, E.; Randall, S.W.; Jones, C.; Forman, W.R. The Mysterious Merger of NGC 6868 and NGC 6861 in the Telescopium Group. Astrophys. J. 2010, 711, 1316, [CrossRef]

390. Pandge, M.B.; Vagshette, N.D.; Sonkamble, S.S.; Patil, M.K. Investigation of X-ray cavities in the cooling flow system Abell 1991. Astrophys. Space Sci. 2013, 345, 183-193. [CrossRef]

391. Blanton, E.L.; Sarazin, C.L.; McNamara, B.R.; Clarke, T.E. Chandra Observation of the Central Region of the Cooling Flow Cluster A262: A Radio Source That Is a Shadow of Its Former Self? Astrophys. J. 2004, 612, 817-824. [CrossRef] 